Article

\title{
Small Scale Problems of the $\Lambda$ CDM Model: A Short Review
}

\author{
Antonino Del Popolo 1,2,3,* and Morgan Le Delliou 4,5,6 \\ 1 Dipartimento di Fisica e Astronomia, University of Catania , Viale Andrea Doria 6, 95125 Catania, Italy \\ 2 INFN Sezione di Catania, Via S. Sofia 64, I-95123 Catania, Italy \\ 3 International Institute of Physics, Universidade Federal do Rio Grande do Norte, 59012-970 Natal, Brazil \\ 4 Instituto de Física Teorica, Universidade Estadual de São Paulo (IFT-UNESP), Rua Dr. Bento Teobaldo \\ Ferraz 271, Bloco 2 - Barra Funda, 01140-070 São Paulo, SP Brazil; delliou@ift.unesp.br \\ 5 Institute of Theoretical Physics Physics Department, Lanzhou University No. 222, South Tianshui Road, \\ Lanzhou 730000, Gansu, China \\ 6 Instituto de Astrofísica e Ciências do Espaço, Universidade de Lisboa, Faculdade de Ciências, Ed. C8, \\ Campo Grande, 1769-016 Lisboa, Portugal \\ * Correspondence: adelpopolo@oact.inaf.it
}

Academic Editors: Jose Gaite and Antonaldo Diaferio

Received: 30 May 2016; Accepted: 9 December 2016; Published: 20 February 2017

\begin{abstract}
The $\Lambda \mathrm{CDM}$ model, or concordance cosmology, as it is often called, is a paradigm at its maturity. It is clearly able to describe the universe at large scale, even if some issues remain open, such as the cosmological constant problem, the small-scale problems in galaxy formation, or the unexplained anomalies in the CMB. $\Lambda$ CDM clearly shows difficulty at small scales, which could be related to our scant understanding, from the nature of dark matter to that of gravity; or to the role of baryon physics, which is not well understood and implemented in simulation codes or in semi-analytic models. At this stage, it is of fundamental importance to understand whether the problems encountered by the $\Lambda \mathrm{DCM}$ model are a sign of its limits or a sign of our failures in getting the finer details right. In the present paper, we will review the small-scale problems of the $\Lambda \mathrm{CDM}$ model, and we will discuss the proposed solutions and to what extent they are able to give us a theory accurately describing the phenomena in the complete range of scale of the observed universe.
\end{abstract}

Keywords: cosmology ; dark matter; small scale problems; $\Lambda$ CDM model

\section{Introduction}

Despite the $\Lambda \mathrm{CDM}$ model being successful, according to the largest part of the cosmology community, in describing the formation and evolution of the large scale structure in the Universe, the state of the early Universe and the abundance of different forms of matter and energy [1-5], its predictive power - already checked against new discoveries (e.g., lensing of the CMB [6,7], B-mode polarisation [8] the kinetic SZ effect) - it presents several difficulties. Among the most famous, we recall the "cosmological constant fine tuning problem", and the "cosmic coincidence problem" $[9,10]$.

The first problem is connected to the fact that most quantum field theories predict a huge cosmological constant from the energy of the quantum vacuum at present, more than 100 orders of magnitude too large, see Refs. [9-11]. More precisely the theoretical expectations give $\rho_{\Lambda} \simeq 10^{71} \mathrm{GeV}^{4}$, in contradiction with the cosmological upper bounds giving $\rho_{\Lambda} \simeq 10^{-47} \mathrm{GeV}^{4}$ which gives rise to an extreme fine-tuning problem. It also entails fine tuning at Planck scale era, thus in the initial conditions of dark energy. The second is connected to the reason why dark energy and dark matter energy densities are approximately equal nowadays (see Ref. [12]).

Tensions of unknown origin are also present between the 2013 Planck parameters [13] and $\sigma_{8}$ obtained from cluster number counts and weak lensing, the actual value of the Hubble parameter, 
$H_{0}$, and SN IA data. The Planck 2015 data are still in tension with CFHTLenS weak lensing [14] data, and with the $\sigma_{8}$ growth rate [15].

Another concern lies in that the large-angle fluctuations in the CMB show statistical anomalies (i.e., a quadrupole-octupole alignment [16-20], a power hemispherical asymmetry [21-26] and a cold spot [27-29]). This collides with the idea that our universe should be a realisation of a statistically isotropic and Gaussian random field, which implies a statistical independence in the CMB multipoles. What is unclear is whether these anomalies are related to unknown systematics, if they are statistical effects [30], or a fingerprints of new physics.

The $\Lambda$ CDM model also encounters problems in describing structures at small scales, e.g., [31-36]. The main problems are/have been

a. The cusp/core (CC) problem [31,37], designating the discrepancy between the flat density profiles of dwarf galaxies (also coined dwarfs), Irregulars, and Low Surface Brightness galaxies (hereafter LSBs), and the cuspy profile predicted by dissipationless N-body simulations [38-40], despite the fact that the observed galaxies are all of DM dominated types;

b. The "missing satellite problem" (MSP), coining the discrepancy between the number of predicted subhalos in N-body simulations [32,41] and those actually observed, further complicated by the "Too Big To Fail" (TBTF) problem, arising from the $\Lambda$ CDM prediction of satellites that are too massive and too dense, compared to those observed, to hope for their destruction in the history of mass assembly up to today $[34,35]$;

c. The angular momentum catastrophe [42] labelling the angular momentum loss in Smooth Particle Hydrodynamics (SPH) simulations of galaxy formation that gives rise to dwarf galaxies' disks with different angular momentum distributions from those of cold dark matter haloes, in addition to disc sizes that are much smaller in simulated galaxies compared with observed ones [43];

d. The problem of satellites planes, namely the alignment on thin planes of satellite galaxies of the MW and M31, a feature that proved difficult to explain in simulations of the $\Lambda \mathrm{CDM}$ paradigm [44];

e. The problem of re-obtaining the slope and scatter of the baryonic Tully-Fisher relation $\left(M_{b} \propto V_{c}^{4}\right)[45] ;$

The $\Lambda$ CDM model has some other issues discussed in Refs. [46-50], that we shall not consider in our short review. Problems c and e have actually been solved: baryonic models, as discussed in Section 4.1 , have already been proposed to solve the problem posed by SPH simulations baryons angular momentum non conservation in collapse, leading them to typically only retain $10 \%$, and form disks that are too small, compared to real galaxies [51-54] (i.e., the "angular momentum catastrophe", AMC). Those solutions proceeded from feedback effects basically heating the gas , from supernovae explosions [53], using clumps, in addition, to reproduce the correct angular momentum distribution of baryons [55-57] and selective outflows to obtain bulgeless disks [58] (see Section 4.2), or from dynamical friction of baryonic clumps, able to explain all those features at once (see Section 4.3, and Ref. [59]).

The empirical optical luminosity-21 cm line width scaling in spiral galaxies, the Tully-Fisher relation [60], that reflects the gas+luminous mass-rotation velocity scaling, the Baryonic Tully-Fisher Relation (BTFR) [61-69], have been used as a stumbling stone for the $\Lambda$ CDM model by proponents of the MOND Modified Gravity Model ([70], discussed in Section 4.4). However, this claim is less strong after some models and simulations have found a possible solution (see Section 3).

Problem d is characterised in the MW by

- a highly flattened, planar distribution of the satellites in three-dimensional space,

- a common orientation of the satellites orbits, and

- an alignment of the satellites orbits within the distribution plane.

and remains open: if Ref. [71] claimed a resolution in the EAGLE hydrodynamical simulations, the review [72] concluded oppositely. 
The present review will be restricted to the small-scale problems of the $\Lambda \mathrm{CDM}$ model (hereafter SSP $\Lambda$ CDM) connected to the formation of cusps, and to satellites. As we will see hereafter, these issues are strictly connected.

From one side, unified models have shown $[59,73,74]$ that a mechanism which can transform cusps into cores conversely helps the solution of the MSP. Several authors (e.g., [75-77]) noticed that the effects of a parent halo's tidal forces on a satellite depend fundamentally on the shape of the latter. A cuspy profile allows the satellite to retain most of its structure, when entering the main halo. Inversely, for a cored profile, the tidal field of the main halo can easily strip the satellite from its gas and even destroy it in some cases [77]. As a result, such satellite will not end up visible, either because it was destroyed or because it lacks the gas to make stars.

From the other side, the satellites most puzzling issue is now recognised as having shifted: rather than the number of satellites, it is related to their inner mass density, specifically to their density profiles being flatter than those of $\mathrm{N}$-body simulations.

In Sections 2, and 3, we will summarise the discussions around, respectively, the satellite plane problem and the Baryonic Tully-Fisher Relation. In Section 4, we will review the CC problem. We will shortly review the early discussions on that problem, then the solution related to the role of baryonic physics, as well as solutions dealing with changes of the dark matter properties or related to modified theories of gravity. In Sections 5, and 6, we review the MSP, and the Too-Big-To-Fail (TBTF) problem. In Section 7, we will discuss a unified baryonic solution to the quoted problems, and Section 7 is devoted to conclusions.

\section{The Satellite Plane Problem}

From the seventies on, it was noticed by several authors that the MW satellites are distributed on a planar structure, the so-called "Magellanic Plane" [78,79], the disc of satellites [46,80], the MW "Vast popular structure" (VPOS), and the "great plane of andromeda" (GPoA) $[44,72,81,82]$. Recent studies by Refs. $[46,81,83]$ described the structure as a thin disc, having a height of $20 \mathrm{kpc}$, and 9 of the 11 classical dwarfs of the MW [80] co-orbit within the structure. Other authors [83-85] found a similar distribution around M31, which was better evidenced by Refs. [82,86], who found the GPoA, (see [87-94]). In the local group (LG), similar alignments have been found in isolated dwarf galaxies [81,95-97], as well as in more distant galaxies [98-101]. According to several authors (e.g., [46]), this should be considered as a strong challenge for the $\Lambda$ CDM model ${ }^{1}$. In cosmological simulations the DM sub-haloes are isotropically distributed and then at odds with the planar VPOS. The VPOS and GPoA structures, studied from the point of view of space correlation led Ref. [83] to conclude on the tidal origin (i.e., tidal dwarfs) of a fraction of the MW and M31 [102,103]. The structure of the observed dwarf galaxies could have been formed from encounters in the LG (e.g., a past encounter between the MW and M31 [104-106], the tidal disruption of a LMC-progenitor $[78,79,107]$, etc). The tidal dwarf galaxies (TDG) scenario has shown to be able to reproduce structures such as the GPoA [93], or VPOS-like structure [108,109].

Several trials have been proposed to obtain the quoted structures in the $\Lambda \mathrm{CDM}$ model. Refs. [110,111] proposed a scenario of accretion of groups of satellites, but this approach gives rise to properties of groups of dwarfs in disagreement with observations [112], as the structures formed are too extended, and too thick ( $\simeq 50 \mathrm{kpc}$ ), in comparison with the VPOS and GPoA. The VPOS structure was claimed to be reobtained in simulations such as that of Ref. [113], but an analysis of the angular momenta directions (orbital poles), by Ref. [105] showed a disagreement with those of observations. This issue was also studied in the simulations by Refs. [114,115]. The first group of authors found that for at least 3 out of 11 satellite galaxies - the latter representing about $35 \%$ of the simulated

1 It was pointed out, however, for the first time by Kroupa et al. [46], that the planar distribution of all known satellite galaxies in 2005 was in significant disagreement with the expected spatial distribution of dark-matter dominated satellite galaxies 
satellite galaxy systems they consider in their $\Lambda$ CDM simulations - the orbital poles point along the short axis of the galaxy distribution. Similar alignment is also claimed by Ref. [115]. Futher improved studies by Ref. [81] found the distribution of orbital poles of the 11 brightest MW satellites to be much more concentrated, displaying a good alignment for $\geq 6$ of the orbital poles, which seems to be difficult to explain for $\Lambda \mathrm{CDM}$. Another way to solve the puzzle is to claim that, since the GPoA-like structures are rare [116], there is no contradiction between the $\Lambda$ CDM Millennium II simulations and observations, as VPOS in MW are as frequent in the Millennium II simulation as in observations [117]. Finally, the paradigm of cold mode accretion streams [118] could naturally explain the GPoA, as, in it, host galaxies acquire not only their gas but also their satellites along cold streams: therefore GPoA would represent indirect observational evidence of the paradigm.

Such claims were criticized by several authors (see [72]), which point the finger at the choice of the parameters used for the comparison.

More recent simulations (e.g., [119]) claim that the problem of reproducing those structures is merely due to neglecting the Zolotov effects of baryons, the comparison being made with $\mathrm{N}$-body only simulations, while when taking those effects into account, such structures are no-longer problems.

Ref. [72] rejected such claims, since the scale on which baryon physics can act is much smaller than that of the satellite systems (some hundreds of kpc)

To summarize, the issue is still open, and from our point of view, should a solution be found, it is highly improbable that it would be connected to baryonic physics.

\section{The Baryonic Tully-Fisher Relation}

The Tully-Fisher relation was discovered to empirically correlate spiral galaxies luminosities with their HI line-width [60], that is their stellar mass with their rotation velocities [61,120,121]. This correlation is well fitted by a power law with small scatter for late-types of high mass and velocities [61,120], while it no longer follows a single power law at low mass and velocities because cold gas starts to contribute significantly. It has therefore been generalised by using baryonic mass instead of just stellar mass, and the resulting Baryonic Tully-Fisher Relation (BTFR) fits well with a single power law over several orders of magnitude [67] and with small scatter for selected high-quality data $[70,122]$.

The concordance cosmology framework understands the BTFR as an imprint of the finite age of the Universe on the halo mass-rotation velocity relation (see, e.g., [123,124]), that translates into a constant density contrast, and thus implies linear scaling of virial radius with velocity, i.e., from geometry, $M \propto V^{3}$. The problems for the $\Lambda$ CDM model coming from the scatter and slope of the BTFR highlighted by [122] (and references therein) hinge on that relation. The link with the BTFR is made by assuming proportionality of galaxies baryonic mass and rotation velocity with their host haloes total virial mass and velocity, respectively. The latter assumption is not trivially satisfied since the observations used for the BTFR clearly provide a very limited baryonic mass and velocity probe compared with equivalent haloes in simulations [125]. In fact, so few halo baryons assemble in actual galaxies that the correlation with virial mass is unclear, while, as the disk sizes remain so small compared to the estimated virial sizes of galaxy haloes, scaling between their two characteristic velocities appears unrealistic. In addition, baryonic feedback mechanisms determining the galaxies' baryonic masses seems too variable to ensure similar galactic baryon fractions for all haloes. The tightness of the observed BTFR is therefore difficult to explain in the $\Lambda \mathrm{CDM}$ model, contrary to the fundamental acceleration scale included in the MOdified Newtonian Dynamic (MOND) proposition [70]. Indeed, [122] claim that observation of a select sample of disk galaxies, assuming constant stellar mass to light ratio, displays a significantly lower scatter than in the $\Lambda$ CDM simulations of Ref. [126], and that the residuals correlations with the radius or surface brightness of galaxies are not following the $\Lambda$ CDM semi-analytic predictions of Ref. [127], a conclusion which puts the $\Lambda$ CDM model at odds with observations. 
For many years, numerical galaxy formation simulations were unable to produce morphologically realistic galaxies, let alone reproduce the BTFR (see, e.g., [128,129], and references therein), and even semi-analytic models, with empirical inputs, had a hard time predicting it correctly (e.g., [130]). Recently, however, this state of affairs has evolved with improvements in modeling of the baryonic feedback mechanisms allowing production of realistic rotation disk galaxies [131-135]. The small statistics of these models did not allow for predictions on the BTFR, while attempts at reproductions led to controversy on the impact of those baryonic processes on the dark halo, some claiming drastic feedback was needed to obtain the BTFR $[136,137]$ while others reported no need for such feedback (see, e.g., $[138,139])$.

Recently, the combination of the large EAGLE simulation programme $[140,141]$ — calibrated on small scales on observed galaxy stellar mass function and present radius but not on the BTFR, with multiple realisations of Local Group-like galaxies in smaller volume, i.e. the APOSTLE project $[119,142]$ — claimed to have successfully reproduced the BTFR over four decades [143], even reproducing its break-down at the faint end, as was indeed observed $[68,144]$.

This, after the other claim of successful model from Ref. [145] (a semi-empirical model coupling observed Halo Abundance Matching baryon mass fractions with $\Lambda \mathrm{CDM}$ haloes and claiming to generate a realistic BTFR), seems to have found a path to solve the problem.

\section{The Cusp/Core Problem}

Flores \& Primack [37] and Moore [31] ruled out cuspy profiles from DDO galaxies' rotation curves, and showed them to be well approximated by cored (or pseudo-) isothermal density profiles. The problem then lies in the cuspy profiles produced in dissipationless simulations of the CDM model (see Figure 1).

The dissipationless N-body simulations of Navarro, Frenk, \& White [38,146], then showed that DM profiles are cuspy, with inner density $\rho \propto r^{\alpha}$, with inner density power index $\alpha=-1$, that they are universal in dissipationless simulations, that is independent from the cosmology, and from the scale (coined NFW profiles). An even steeper profile predicted by $[147,148]$ gave $\alpha=-1.5$, while other authors found that the inner slope is dependent on the object considered, and/or its mass [149-157]. More recent N-body dissipationless simulations tend to agree on the fact that a profile flattening towards the centre to a minimum value of $\simeq-0.8$ [158], namely the Einasto profile, seems to give a better fit to simulations [159] (see Figure 2). The problem lies in the fact that the smallest value predicted by dissipationless N-body simulations is larger than the values obtained respectively by observations [36,160-164], in SPH simulations [58,165], or in semi-analytical models [166-172].

That discrepancy has been fervently debated for two decades. Early HI observations of LSBs led to contradicting results. McGaugh \& de Blok [173] found a discrepancy between their Rotation curves (RCs) and the NFW halo, as did Cote et al. [174]. Conversely, van den Bosch \& Swaters [175] could not exclude steep profiles in several of the objects they studied. Similar contradictions were obtained using $\mathrm{H} \alpha$ observations: de Blok \& Bosma [176] found evidence against steep profiles, de Blok et al. [161] measured inner slopes $\alpha=-0.2 \pm 0.2$, and similarly Spekkens et al. [177] obtained $\alpha=-0.22 \pm 0.08$, while Hayashi et al. [178] showed RCs in agreement with cuspy profiles. High resolution observations usually agreed on flat profiles ([179-183], etc.).

Gentile et al. [184-186] decomposed the total rotation curves of some spiral galaxies in stellar, gaseous, and dark matter components. Fitting the density with various models they found that constant density core models are preferred over cuspy profiles. Similar results were obtained by Oh et al. [36] using 7 dwarf galaxies from THINGS (The HI Nearby Galaxy Survey) galaxies. The comparison of the RCs (and the density profiles) with the NFW, and pseudo-isothermal (ISO) profiles is plotted in Figure 3. The plot clearly shows that the ISO profile is a better fit to the RCs. 

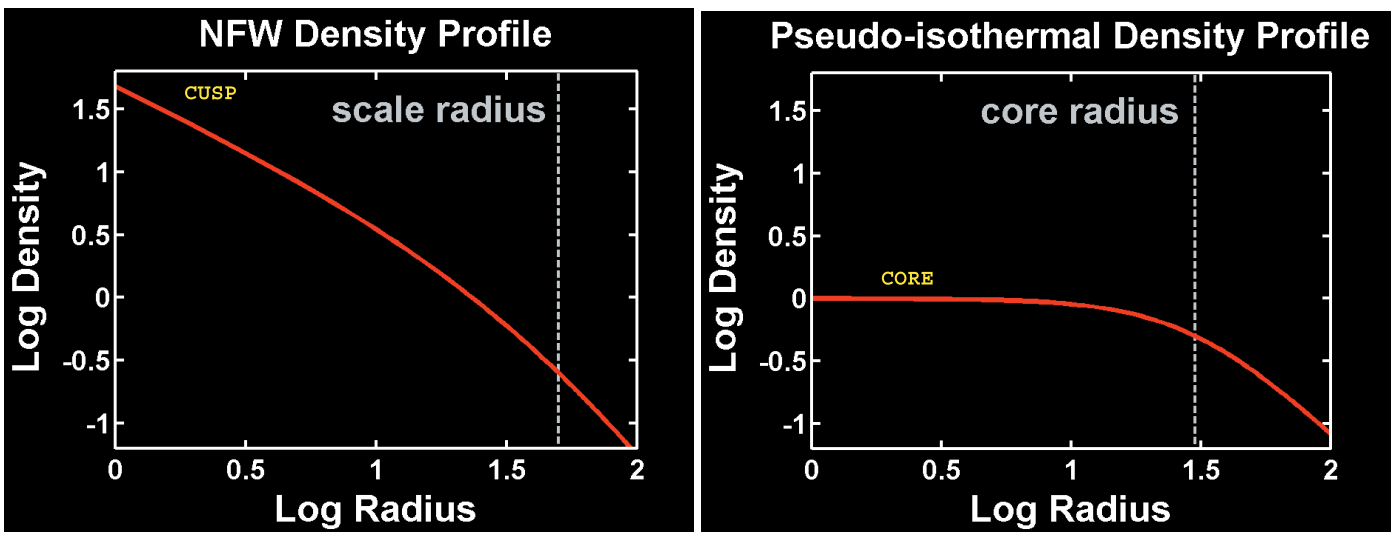

Figure 1. Schematic representation of the CC problem. The left panel represents the cuspy NFW profile. The dotted vertical line is the scale radius of the profile. The right panel represents the case of a cored profile. The dotted vertical line is the core radius of the profile.

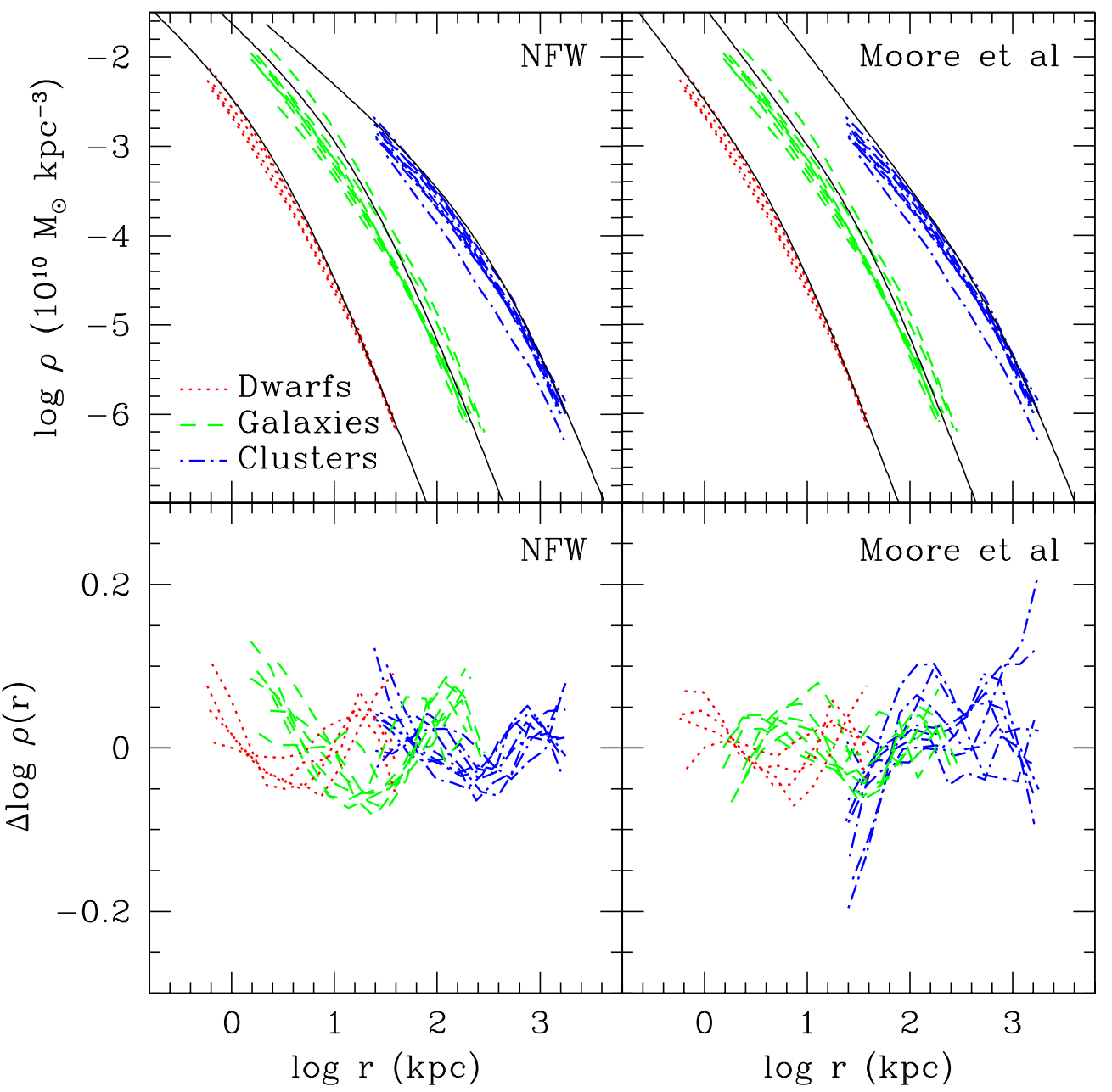

Figure 2. Density profiles comparison. The top left corner is a comparison of the NFW profile (solid line) with Ref. [187]'s simulations, that are well fitted by the Einasto profile [39], in the cases of dwarfs (red line), galaxies (green line), and clusters (blue line). The top right corner is the same as top left, but for the Moore profile. The bottom left and right corners display the residuals (Figure reproduced from [187]). 

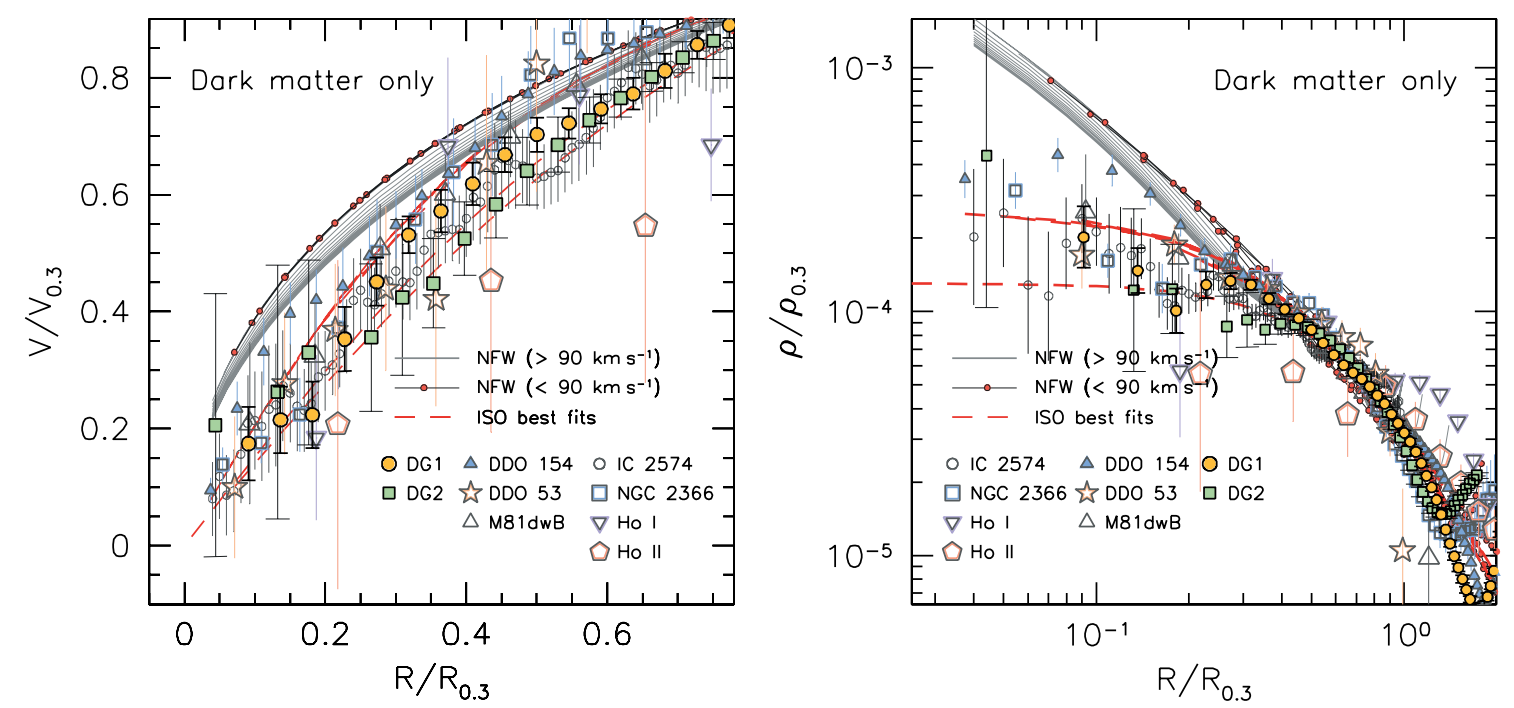

Figure 3. Left panel: Comparison of the RCs from (a) 7 dwarf galaxies from THINGS; (b) the two galaxies DG1 and DG2 simulated by [58], to the NFW (solid lines), and pseudo-isothermal (ISO) profiles (red dashed line). The NFW RCs with $V_{200}$ in the range $10-90 \mathrm{~km} / \mathrm{s}$ are highlighted by small red dots. The rotation velocity $V$ is scaled to $V_{0.3}$, namely the value of $V$ at $R_{0.3}$, representing the distance at which $\frac{d \log V}{d \log R}=0.3$. Right panel: same as the left, but for the density profiles (figure reproduced from [36]).

Similar results were also presented using the LITTLE THINGS galaxies [188].

These studies were mainly related to dwarf or low surface brightness galaxies. In the case of high-surface brightness objects, or large galaxies, determining their inner density structure is more complicated. Therefore, stating the nature of the inner density profile for all galaxies, cored or cuspy, is not so obvious nowadays. While, according to [189], high-surface brightness galaxies are cored, other authors (e.g., [156,170,190-192]) conclude differently. The THINGS sample tends to be better described by isothermal profiles (ISO) for low luminosity galaxies, $M_{B}>-19$, while for $M_{B}<-19$, cuspy or cored profiles describe them equally well.

The situation is even more confusing as dwarfs do not always have flat slopes, as seen in Simon et al. [190]. They studied the low mass spirals NGC2976, NGC6689, NGC5949, NGC4605, and NGC5963, where they found a large scatter in the inner slope $\alpha$ : for NGC2976, they obtained $\alpha \simeq 0.01$, compatible with a cored profile, while for NGC5963 they got a cuspy one, $\alpha \simeq 1.28$. The other three galaxies had $\alpha \simeq 0.80$ (NGC6689), $\alpha \simeq 0.88$ (NGC5949), and $\alpha \simeq 0.88$ (NGC4605).

In Figure 4, the top left panel plots the DM halo RC of NGC5963 (black dots with error-bars), together with the RC obtained from a fitted NFW profile (cyan line), from a fitted pseudo-isothermal profile (ISO, short-dashed magenta line), and from the model of [166] (yellow dashed line) taking account of baryonic physics. The top right, and bottom panels display the same data for the cases of NGC5949, and NGC2976, respectively, with the same kind of fitted profiles for NGC5949, and just a flat power law (black line) and the same model [166] (dashed line) for NGC2976. The cuspy density profile of NGC5963 is well approximated by an NFW profile, which reflects in the RCs. NGC5949's RC is fitted equally well by RCs from an ISO or an NFW profile, while NGC2976 displays a very flat inner density $(\alpha \simeq 0.01$. All the three RCs are well approximated by the [168] model.

In other terms, if a large part of dwarfs are well described by cored profiles, others are not.

Different results have even been obtained using similar techniques for the same object. For example, the dark matter profile inner slope of NGC2976 is bracketed between $-0.17<\alpha<-0.01$, according to [193], while [194] got $\alpha=-0.90 \pm 0.15$, and [195] found, considering tracers being stars, $\alpha=-0.53 \pm 0.14$, or gas, $\alpha=-0.30 \pm 0.18$. 

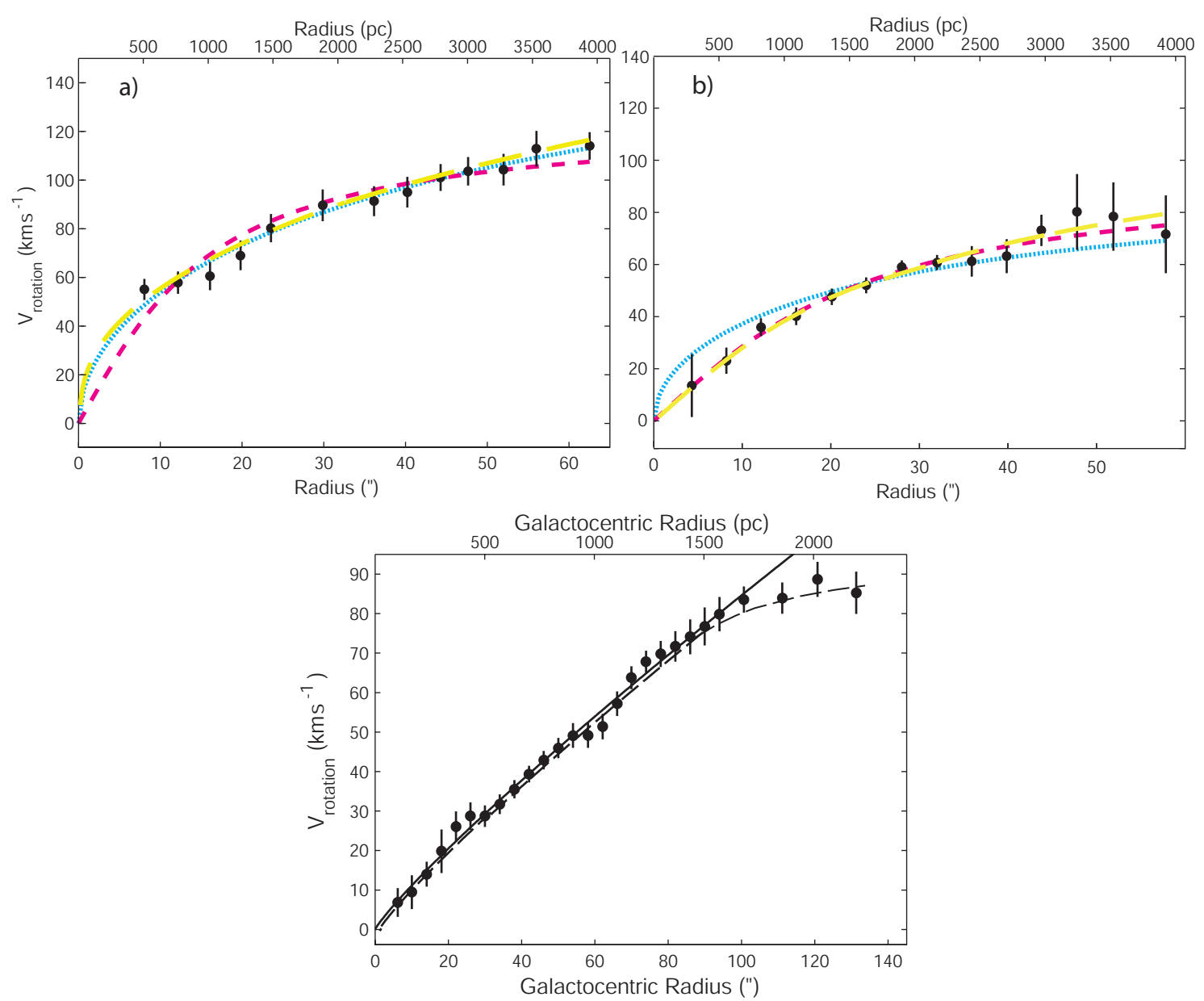

Figure 4. Top left panel: DM halo RC of NGC5963 (black points with error-bars) computed by [190]. The dotted cyan represents the RC obtained from a fitted NFW profile, the short-dashed magenta, from the pseudo-isothermal profile fit (ISO), and the yellow dashed line, from the model of [168]. Top right panel: same as the top left panel, for NGC5949. Bottom panel: DM halo RC of NGC2976 (black points with error-bars) obtained by [190]. The solid line is the RC computed from a power-law fit to the corresponding density (slope $\simeq 0.01$ ), and the dashed line, the RC obtained by the model of [168] (from whom this figure is adapted).

Somehow in agreement with the previous discussion, [196] found that the shapes of observed rotation velocity of galaxies display a much larger variation than in simulations.

The discussion above highlights that the determination of the inner slope of galaxies, even for dwarfs, is no easy task. Moreover, it points out that the CC problem must be defined in terms of the inner mass of galaxies rather than of the inner slope of RCs or density profile. The result from the studies discussed above, and of several others, shows the existence of a range of profiles, and that, even with the improvements of nowadays kinematic maps, there is no agreement on the exact dark matter slopes distribution based on morphologies [36,190,195].

The situation is even more flagrant going to larger masses (e.g., spiral galaxies) dominated by stars, or especially to smaller masses (e.g., dwarf spheroidals (dSphs)) where biases that enter in the system modelling lead to opposite results.

Several techniques have been used to understand and evaluate this problem on dSphs. The spherical Jeans equation gives results highly dependent on the assumptions, since mass and anisotropy 
of the stellar orbits are degenerate in such model [197]. Maximum likelihood in the parameter space approach applied to Jeans modelling [198-200] is plagued by similar such degeneracies. Schwarzschild modelling has been applied to, e.g., Sculptor and Fornax dSphs, finding cored profiles [201-204]. Methods based on multiple stellar populations concluded that Fornax (slope measured at $\simeq 1 \mathrm{kpc}$ ) and Sculptor (slope measured at $\simeq 500 \mathrm{pc}$ ) have a cored profile [205-208]. However, a cusp is found in Draco using a Schwarzschild model [204]. This latter results show that in reality there is no accepted conclusion on a unique inner structures of dSphs.

A similar problem is also present in galaxy clusters. Sand et al. [209] combining weak lensing, strong lensing, and velocity dispersion studies of the stars of the BCG (Brightest Central Galaxy) found that out of the clusters MACS1206, MS2137-23, RXJ1133, A383, A1201, A963, only RXJ1133 had a profile compatible with the NFW model, and similar studies of Newman et al. [210] (for A611), Newman et al. [211] (for A383), and Newman et al. [212] (for MS2137, A963, A383, A611, A2537, A2667, A2390) (see also [213]) also found flatter profiles than other studies. For example, Donnaruma et al. [214] found a cuspy profile for A611 combining strong lensing and X-ray observations, among other discrepancies from Newman's [212], which covered seven relaxed, massive clusters with flat and cuspy profiles and an average slope $\alpha=0.50 \pm 0.1$.

In general gravitational lensing yields conflicting estimates: they sometimes agree with numerical simulations [214-216] but can also find much shallower slopes (-0.5) [209-212,217-219]. X-ray analyses have similarly led to a wide range of slope values, from -0.6 [220] to -1.2 [221] till -1.9 [222], but can also agree with the NFW profile [223].

While early observations obtained conflicting results concerning the inner structure of the density profiles, high resolution observations, on average, agree on profiles flatter than the NFW's. At the same time, the new observations show a diversity in the inner structure from galaxy to galaxy, as also shown in [196] simulations.

Even if [196]'s simulation results (shaded green band in Figure 5) are in agreement with the RC of galaxy IC2574 at radius $>6 \mathrm{kpc}$, their behaviour in the inner parts is completely different. This discrepancy points out that convergence in the inner slope of RC between simulations does not mean that they are correctly describing the whole behaviour of the RC. In fact, the deficit of the mass in the inner part of the profile better characterises the CC problem [196]. The key issue is not the shape of the density profile but the excess amount of DM predicted by CDM in the central kpcs of the galaxy. The tension is already evident at scales at which the circular velocity reaches its asymptotic value [224].

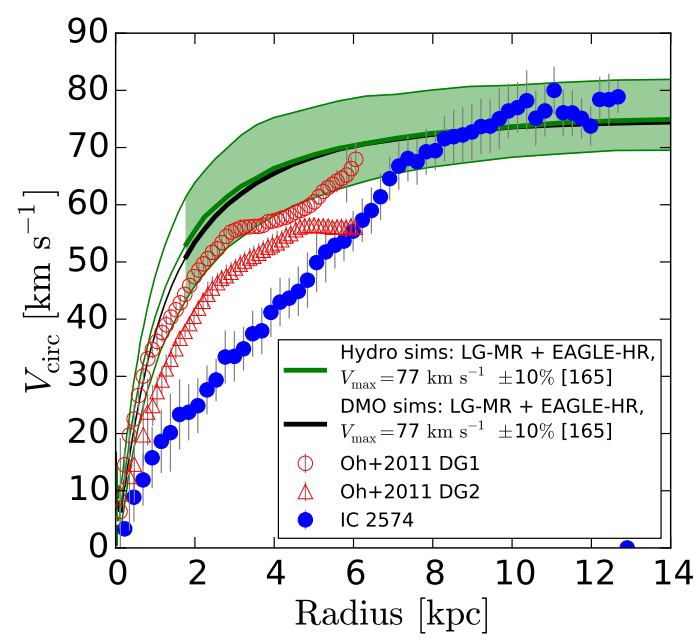

Figure 5. Comparison of the observed IC2574 RC (filled circles) with the two simulated galaxies DG1, and DG2 of [58]. The green line, and shaded region, represent respectively the median rotation velocity, and scatter, of the galaxies simulated by [196] (from which this figure is reproduced). 
Unfortunately, almost all the observation papers in the literature estimate the inner slope of galaxies through $\alpha$, except for [206] who measured the inner slope through $\Gamma \equiv \Delta \log M / \Delta \log r<3-\alpha$ for Fornax and Sculptor. The latter slope, an integrated quantity, is more easily evaluated than the local quantity $\alpha$, but only provides some constraints, not a precise value for the inner slope.

On the other side of the mass spectrum, a clear determination of the inner structure of dSphs, cored or cuspy, is very important, as objects with smaller masses are more likely to display a similar inner profile than that of dissipationless N-body simulations predictions (cuspy).

\subsection{Early Solutions}

Many solutions have been proposed to solve the CC problem and in general the SSP $\Lambda$ CDMs. A decade ago some authors (e.g., Refs. $[175,225]$ ) turned against observations, claiming that the inconsistencies could be due to poor resolution or to an improper account of systematic effects. Non-circular motions, beam smearing, off-centring, slit-misplacement, which tend to systematically lower slopes, were charged with the discrepancies.

In $\mathrm{HI}$ observations, the finite beam size produces a smear out of $\mathrm{HI}$ emission, giving rise to larger disks. The effect depends on the size of the beam, the HI distribution, the inclination angle of the galaxy, and the intrinsic velocity gradients. The problem can be solved with high spatial resolution observations, $<1 \mathrm{kpc}$ (see the following).

In $\mathrm{H} \alpha$ observations a slit misplacement may lead to missing the dynamic centre of the galaxy, with the result of having flatter profiles. The problem can be solved in different ways (e.g., 3d spectroscopy $[180,189])$. The gas is usually assumed to move on circular orbits, so non-circular motions produce an underestimation of the slope. Those motions are, however, of the order of a few $\mathrm{km} / \mathrm{s}$, as shown by [183]. Nowadays high resolution observations can distinguish cored and cuspy haloes by deriving their asymptotic inner slopes from rotation curve data [163].

Several authors $[178,187,226]$ suggested ways to reconcile simulations with observations, claiming their simulations were in good agreement with observations, since they become progressively shallower from the virial radius inwards, and that the discrepancy came from projection effects. Indeed, DM haloes are 3-D, but the practice is to compare spherically averaged circular velocity of DM haloes with the rotation speeds of gaseous disks. In other words, the observational disagreement would be with the fitting formulae, rather than with simulated haloes [178].

Nowadays, this proposal is easily rejected: high resolution DM-only simulations have a minimum inner slopes $\simeq-0.8$ [158], while the inner slope of galaxies observed with high resolution techniques are much smaller.

Another possibility took seriously the failure of the CDM model, claiming the problems were with the simulations $[161,227,228]$. However, modern simulations do not suffer from their past problems: lack of resolution, relaxation, and over-merging. Already then, convergence tests performed by [229] showed that N-body simulations correctly determine the CDM density profiles. The N-body simulations used in the past were dissipationless, meaning they only took account of DM, while baryons are not negligible in the inner regions of galaxies (inner $\mathrm{kpc}$ ), and dominate over DM in the central $10 \mathrm{kpc}$ of clusters [209-212]. Nowadays, high resolution cosmological hydrodynamic simulations are available and we will discuss their important role in the next sections, dealing with the baryon solutions of the SSP $\Lambda$ CDM model.

Pushing further, the validity of the CDM paradigm was questioned, so it was speculated that other forms of DM (warm [230], fuzzy [231], repulsive [232], fluid [233], annihilating [234], decaying [235], or self-interacting [236]) could solve the SSP $\Lambda$ CDM. More radical alternative solutions modified the spectrum at small scales (e.g.,[237]), or gravity (e.g., $f(R)[238,239], f(T)$ theories - see [240-243], or 
the Modified Newton Dynamics - MOND, [244,245]) $)^{2}$. In what follows, we will denote those paradigm changing solutions, whereas for DM, initial conditions or gravity, as "cosmological solutions".

\subsection{Baryonic Solutions to the CC Problem}

As discussed above, the CC problem could be solved with "cosmological solutions" that do not preserve the $\Lambda \mathrm{CDM}$ model, also known as the concordance model. Such modification could, however, alter the successful predictions of the concordance model, that explains many of the observations and features of our Universe.

Thus, before throwing away such a model, it would be wise to verify if some piece of poorly understood or neglected local physics could be connected to the small scale problems.

$\Lambda \mathrm{CDM}$ solutions of the CC problem are based on "astrophysical solutions", for which some mechanism, "heating" the DM, would produce an inner flatter density profile, such as

a. the effect of a rotating bar;

b. transferring angular momentum (e.g., from baryons to DM) through dynamical friction $[166,247,248]$;

c. AGN feedback, gas bulk motions generated by supernova (SN) explosions $[58,75,76]$;

d. the presence of a central black hole giving rise to a shallower cusp, as claimed by some authors [249-251];

e. the role of angular momentum in structure formation.

Several authors used spherical [252-263], or elliptic [264] infall models ${ }^{3}$, arriving to the conclusion that the larger the angular momentum of a proto-structure, the flatter its inner density profile, and finding agreement with the rotation curves of dwarfs [256]. Since the angular momentum acquired by a structure is anticorrelated with the peak height ${ }^{4}$, the density profile of dwarfs is flatter than that of giant galaxies.

El-Zant et al. [247,248] showed how clumps of baryons lose energy, transferred through dynamical friction to the DM component of the system, flattening or erasing the natural DM cusp, both in dwarf galaxies and clusters of galaxies. Other authors studied adiabatic contraction of DM haloes $[270,271]^{5}$, that conversely produces a steepening of the density profiles.

Del Popolo (see also [273]) took simultaneously into account these effects [166]:

- ordered angular momentum acquired by the proto-structure through tidal torques;

- random angular momentum;

- energy and angular momentum exchange between baryons and DM through dynamical friction;

- $\quad$ and adiabatic contraction.

Angular momentum, and dynamical friction in galaxies and clusters not only tend to flatten their density profiles but also to change their global structure [274-277].

Ref. [166] showed that comparing dissipationless simulations with real structures containing baryons is not correct: the role of baryons in the inner part of the proto-structure is not negligible, explaining the discrepancy between N-body simulations and observed density profiles. In Figure 6, the evolution of haloes of $10^{9}$, and $10^{14} M_{\odot}$ are shown. Ref. [166] inscribed itself in the "Dynamical Friction from Baryonic Clumps" scenario (hereafter DFBC scenario) discussed in the following.

$2 f(R)$, and $f(T)$ theories are types of modified gravity theories, generalisation of Einstein's General Relativity. First proposed in 1970 by Buchdahl [238], and turned into an active research field by Starobinsky [239], $f(R)$ theories are defined by a different function of the Ricci scalar in their Lagrangian [246]. Inspired by the Teleparallel Equivalent of GR, the $f(T)$ theories have been introduced to explain Universe acceleration without using dark energy (see Ref. [240]). Finally, the Modified Newtonian Dynamics, was introduced in 1983 by Milgrom [244,245] as a way to model rotation curves of galaxies.

3 General Relativity secondary infall models have been presented by a group around Mimoso and Le Delliou [265-268].

4 The peak height of a proto-structure is defined as $v=\delta(0) / \sigma$, where $\delta(0)$ is the central peak overdensity, and $\sigma$ is mass variance (see [269]). $v$ is larger for more massive objects.

5 Calculated through iterative techniques (e.g., [272]). 

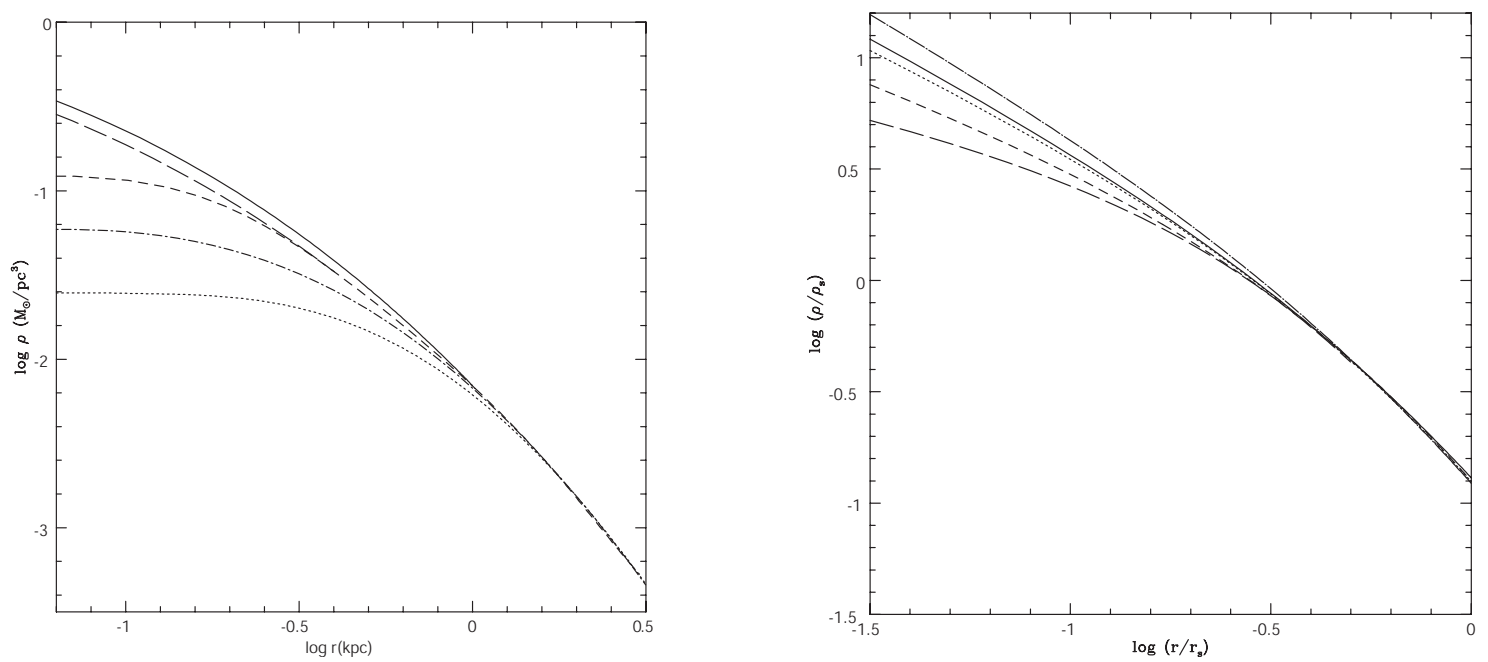

Figure 6. Left panel: evolution of the density profile of a $10^{9} M_{\odot}$ halo [166] (see the discussion on the DFBC scenario). The profile at $z=10,3,2,1$, and 0 , are represented by the solid line, long-dashed line, short-dashed line, dot-dashed line, and dotted line, respectively. Right panel: evolution of the density profile of a $10^{14} M_{\odot}$ halo of [166] (see the discussion on the DFBC scenario). The dot-dashed line represents the total mass density profile (DM+Baryons) at $z=0$, while the DM profile at $z=3,1.5$, 1 and 0 is represented by the solid line, dotted-line, short-dashed line, and long-dashed line, respectively (figure reproduced from [166]).

In Del Popolo [168], the model was applied to dwarfs galaxies showing the influence of the formation history, the content of baryons, and the environment on their density profiles.

A different process was for the first time proposed by Navarro et al. [278], based on supernovae feedback (see the following), that was able to flatten the DM profile.

Currently, the most favoured astrophysical solutions are

a. "supernovae feedback flattening" (SNFF) of the cusp [58,75,76,165,278-280], and

b. "dynamical friction from baryonic clumps" (DFBC) $[166,247,248,281-287]$.

Before discussing those mechanisms, it is useful to recall how baryons and DM can "interact".

Smoothly distributed baryons with DM give rise to the adiabatic contraction (AC) of DM and baryons collapse towards the galactic centre, which steepens the DM profile and thus increases the central density of the structure. However, the effects of AC can be counteracted if energy is transferred from baryons to DM. This can happen if

a. The orbital energy of incoming clumps is transferred to DM through dynamical friction. As a consequence, DM particles move to the outskirts of the galaxy, and the density DM profile is flattened [166,247,248,281-287] (see also the review of [288]).

b. Internal energy sources in the galaxy [288] heat up the DM particles.

If baryonic matter is expelled from, or even moved in, the galaxy (bulk motions produced by supernovae explosions, $[75,76])$, this produces a temporary flattening of the gravitational potential, moving DM particles outwards, and flattening the cusp.

\subsection{Supernovae Feedback Flattening}

Since the suggestion from Ref. [37], stressed in many following works, of the importance of baryons in solving the CC problem, the first mechanism envisaged was connected to supernovae feedback $[58,75,76,119,165,278-280,289]$.

Ref. [278] showed that the sudden expulsion of baryons into the halo in a single event could flatten the profile. The process is most efficient for galaxies with shallow potential, such as dwarfs. 
However, Ref. [290] showed that, while a single explosive event did not move sufficient energy to form a core, repeated moderately violent explosions could reach the goal (however see [291] for a different point of view).

Gelato and Sommer-Larsen [279] studied more in detail the [278] scenario, trying to reproduce DDO154's RC starting from NFW profiles: they tried to reproduce a gas outflow event by abruptly changing the disk potential. They found that it was necessary to expel at least $75 \%$ of the disk mass in order to reproduce the RC .

Read and Gilmore [280] showed that repeated outflows followed by gas re-accretion, could give rise to a core even in larger galaxies.

Refs. $[75,76]$ showed that random bulk motions of gas driven by SN explosions in primordial galaxies could form a core. Similar results were obtained in [58] simulations. Refs. [36,164] compared the average slope of THINGS dwarfs with the simulations by [58]. Governato et al. [165] ran simulations to study galaxies larger than in [58], and compared the results with observations. They found, for $M_{*}>10^{7} M_{\odot}$ galaxies, a correlation between the stellar mass $M_{*}$ and the inner slope.

Governato's papers used the code GASOLINE [292], a N-Body+SPH code to simulate galaxies. By means of the "zoom" technique [293], the resolution for gas particles was brought down to $M_{\mathrm{p}, \text { gas }}=3 \times 10^{3} M_{\odot}$, while the DM particles had $M_{\mathrm{p}, \mathrm{DM}}=1.6 \times 10^{4} M_{\odot}$, and the softening retained at $86 \mathrm{kpc}$. The authors performed a two runs with different star forming thresholds: one in which stars formed if the hydrogen density was $>100 / \mathrm{cm}^{3}$ (High Threshold run, HT), and another with hydrogen density threshold $>0.1 / \mathrm{cm}^{3}$ (Low Threshold run, LT). These simulations, similarly to [157], implemented the blast wave SN feedback mechanism [294], and/or early stellar feedback [295]. There, the interstellar medium (ISM) received $10^{51}$ ergs of energy from $>8 M_{\odot}$ stars. Energy ejected from SN ejected energy was coupled with the coefficient $\epsilon_{\text {esf }}$ to the ISM. The MaGICC simulations [295] adopted the fiducial $\epsilon_{\text {esf }}=0.1$.

Similarly, [296] showed that final result of cusp flattening was generated by combining bursty star formation together with supernovae feedback, resulting in fast oscillations of the inner $(1 \mathrm{kpc})$ galaxy potential, and expanding gas bubbles. This process only starts after the galactic centre accumulated cold gas density reaches $>100 / \mathrm{cm}^{3}$ and stars form ${ }^{6}$. Smaller densities (e.g., $0.1 / \mathrm{cm}^{3}$ ) do not produce any visible changes in the DM inner density profiles. Governato et al. [165], repeated the calculation for larger mass galaxies (see Figure 7, top left and right panels). Teyssier et al. [289] used the adaptive mesh refinement code RAMSES together with a new SN feedback scheme, finding results in agreement with [296] (see Figure 7, bottom left panel), which showed that $M_{*}>10^{7} M_{\odot}$ galaxies have a flat inner profile. Onorbe et al. [298] found similar results, but for $M_{*}>10^{6} M_{\odot}$ galaxies (Figure 7 bottom right panel) ${ }^{7}$.

This view was recently criticised by [196]. According to their simulations, in systems having $V_{\max }<60 \mathrm{~km} / \mathrm{s}$, baryons have little effect on the rotational curve even in the inner regions of the galaxy.

According to [196], the cores formed in [296] are fundamentally related to an ad hoc choice of parameters, while in their own simulations had no evidence of core formation.

Those results are in agreement with that of Ref [299]. The latter group simulated 7 high-resolution dwarfs, living in $1-2 \times 10^{10} M_{\odot}$ mass halos, with different assembly history. They found no case of flattening of the inner core. Their lowest inner slope (at $0.01-0.02 R_{\mathrm{vir}}$ ) was -0.8 and corresponded to a dwarf formed in a halo with a very extended assembly history, which also implies a more extended star formation rate (SFR) history. In addition, [139] got realistic galaxies in simulations, but formed no cores.

6 Consistent with [58] assumptions; the threshold $>10 / \mathrm{cm}^{3}$ [297] marks the limit for bulk gas flows.

In general, the supernovae feedback mechanism is not able to transform cusps into cores in galaxies with $M_{*}<10^{6} M_{\odot}$. 

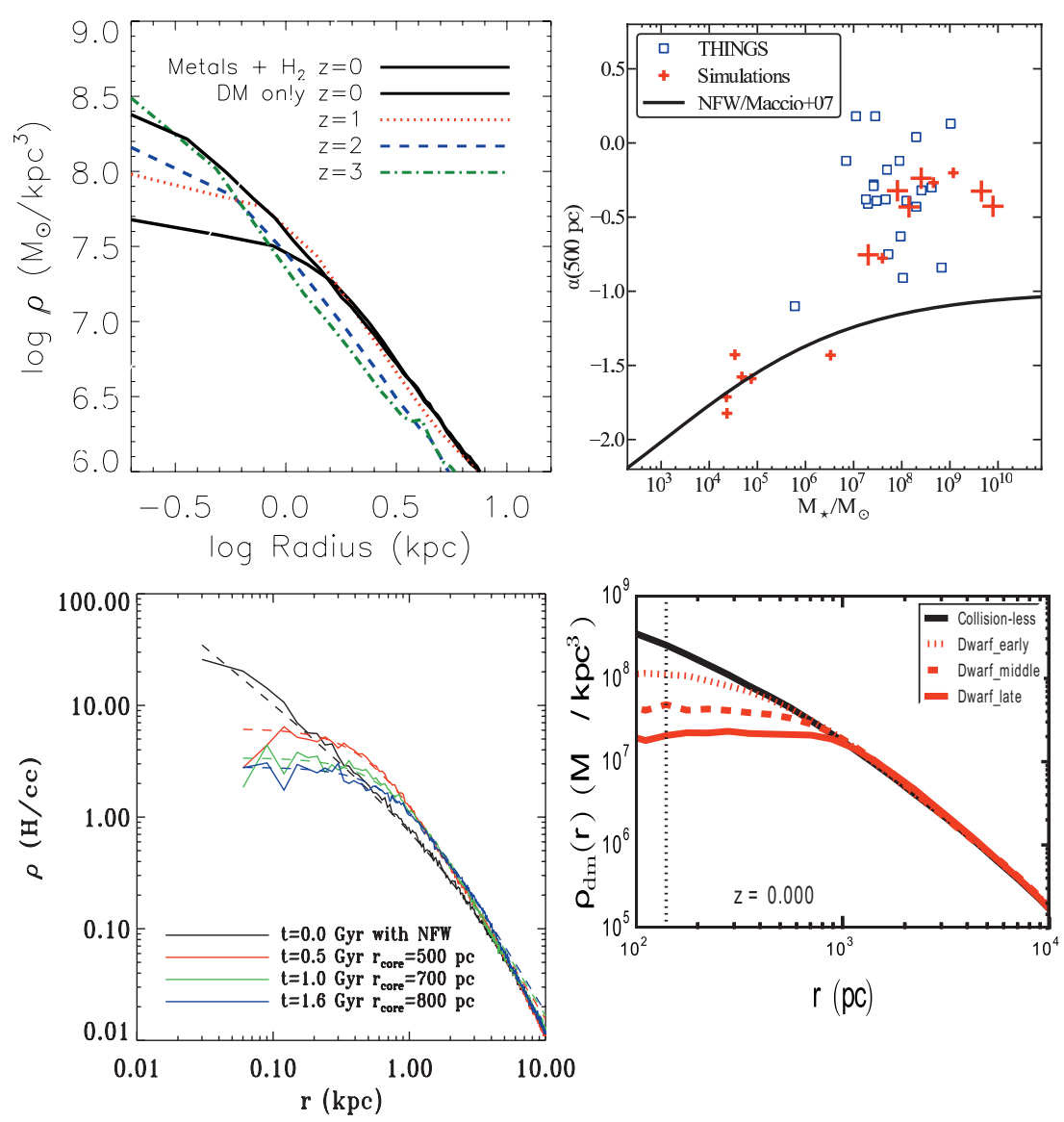

Figure 7. Effect of baryons on density profiles. The top left panel represents the evolution of a density profile in Ref. [165]'s hydrodynamic simulations. The top right panel compares their inner logarithmic slopes (at $0.5 \mathrm{kpc}$ ), for galaxies of different stellar mass (red crosses), with THINGS galaxies (open squares). The solid line represents the result of a previous DM-only N-body simulation [reproduced from 165]. The bottom left panel displays the density profile evolution in the hydrodynamic simulations of [289]. The bottom right panel shows the density profile evolution in the [298] hydrodynamic simulations for three different dwarfs: early (all stars form in early times), medium, late.

Besides the contradicting results on core formation obtained in different high resolution hydrodynamical simulations, the results of $[58,300]$ (and simulations using their same methods and assumptions) have been criticised on several fronts:

a. the energetics of the core formation [301] (galaxies with $M_{*}<10^{7} M_{\odot}$ have too few stars to generate the requested energy to flatten the cusp) and the required baryonic mass, marginally exceeding the baryon content of the dSphs [291]. Figure 8 illustrates that problem in its left panel, from Penarrubia [301], while the right panel is reproduced from Maxwell [302]'s study that arrives at opposite results.

Moreover, while the solution to the CC problem with the SNFF model needs a large number of SNs, and thus a large star formation efficiency (SFE), the solution of the TBTF problem, places an opposite demand on the star formation efficiency (SFE);

b. too high a value of energy coupling, $\epsilon_{\mathrm{SN}} \simeq 0.4$, compared to 0.05 , a value deduced by [303];

c. a very high star formation threshold $[196,304]$ required to obtain the results of [58];

d. they present difficulties in solving the TBTF problem [291,305,306];

e. they lack resolution to follow the feedback processes which should transform the cusp into a core [135,307-309]. 

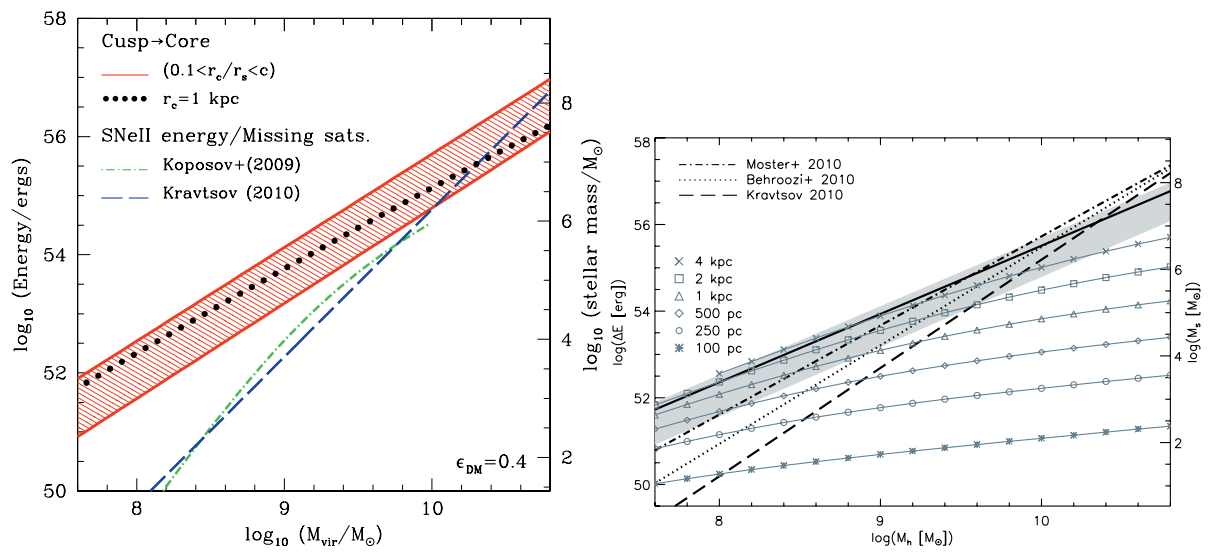

Figure 8. Left panel: $\mathrm{SN}$ energetic output with halo mass. The red shaded area represents the minimum energy of SN explosions, $\Delta E$, needed to generate a core of size $0.1<r_{c} / r_{s}<c, c$, and $r_{S}$ being the concentration parameter and the scale radius of the NFW profile of virial mass $M_{\mathrm{vir}}$, respectively. The dotted black line corresponds to a core of size $r_{c}=1 \mathrm{kpc}$. The right vertical axis displays the luminosity, in stellar mass, derived from a star formation efficiency $F_{*}=F_{*}($ Mvir $)$ constrained by the number of luminous satellites in our Galaxy. Luminosities are converted into SNeII energy using Equation (6) of Ref. [301] and adopting a strong energy coupling $\epsilon_{D M}=0.4$. The outputs of energy found for SN explosion compatible with the "missing satellite" problem in two different studies [310,311]. The tension between the "core/cusp" and "missing satellite" problems becomes obvious in haloes with $M_{\text {vir }}<10 M_{\odot}$ (panel reproduced from [301]). Right panel: Estimates from Ref. [302] of the energy $\Delta E$ required to convert a dark matter cusp into a core. Similarly to the left panel, the left axis shows SN energy outputs. However, the right axis shows the stellar mass corresponding to $\Delta E$ assuming $100 \%$ efficiency $\left(\epsilon_{D M}=1\right)$. The energy required to obtain the pseudo-isothermal density profiles, at given fixed core size, yields the solid lines with symbols. The solid black line shows how $\Delta E$ scales with halo mass $M_{h}$ while the limit radius of redistribution of cusp mass $r_{m}$ scale as a fixed fraction of the halo radius $r_{h}$. The grey area corresponds to the energy estimate of Penarrubia et al. [301], shaded in red in the left panel. The dotted, dashed, and dot-dashed lines display the $M_{s}-M_{h}$ relations of Behroozi et al. [312], Kravtsov [311], and Moster et al. [313] respectively (panel reproduced from [302]).

Refs. [36,164] compared the average slope of THINGS dwarves with the simulations by [58], and [165] made a similar comparison for larger objects, and found a correlation among $M_{*}$ and the inner slope for galaxies having $M_{*}>10^{6} M_{\odot}{ }^{8}$.

Conversely, for $M_{*}<10^{6} M_{\odot}$, hydrodynamic simulations predict cuspy profiles . This result is in conflict with Ref. [206]'s results for Fornax and Sculptor inner structure. They showed that both galaxies are compatible with a core, using the slope of the mass profile $\Gamma \equiv \frac{d \log M}{d \log r}<3-\alpha$ as it gives a more reliable inner slope of DM haloes. Ref. [314] claimed simulations in agreement with [206], injecting however 50\% more energy from SNs.

Gnedin and Zhao [290], considering SN feedback in a Semi-Analytic model based on peculiar assumptions, claimed it cannot produce cores. Without discussing their assumptions, even supposing that they were considering all the details of stellar feedback, their objections are lessened as they did not consider the role of the baryonic clumps on core formation as done in Ref. [166].

The only SNFF simulations forming cores in dwarf galaxies with masses $<10^{6} M_{\odot}$ used the GIZMO code in P-SPH mode [298]. This is much more naturally obtained in the DFBC scenario, that we are going to discuss in the next section. 


\subsubsection{Gas Clumps Merging}

As already reported, the other mechanism able to transform cusps into cores is that proposed by Refs. $[247,248]$. There are three ways, previously discussed, by which DM and baryons can interact. In the case when baryons form clumps of masses $\simeq 0.01 \%$ of the system mass, they can transfer through their motion - their orbital energy to DM. As a result, and similarly to the SN feedback scenario, DM particles will move towards outer orbits, flattening the inner DM profile. The process is more efficient when it occurs earliest, on smallest haloes.

Ref. [247] showed how such mechanism works in galaxies, and [248] how it works in clusters. Ref. [282] used a similar model to study the evolution of the cluster C0337-2522, finding that after the formation of the brightest cluster galaxy (BCG), the inner DM profile has a baryonically induced inner slope, $0.49<\alpha<0.90$, smaller than the NFW profile.

Ref. [283] used N-body simulations, with a hybrid N-body/SPH code, to study the evolution of galaxies in the DFBC scenario. They compared their results between the case of DM-only systems and of mixed systems containing DM and baryons. They found that baryons subhalos heated up the cusp, forming a $\simeq 1 \mathrm{kpc}$ core.

These results were also confirmed in the simulations of Refs. [285-287].

The scenario may be summarised as follows. Initially, the proto-structure contains DM and diffuse gas in the linear phase. DM goes non-linear first and forms the potential well in which baryons will fall.

Clumpy structures form from the instability of accreting gas (e.g. [315-323]) connected to the arising of a very gas-rich disc.

The rotating disc turns unstable and forms clumps when its surface density, $\Sigma$, becomes too large, namely when $Q \simeq \sigma \Omega /(\pi G \Sigma)<1$ [324], with $\Omega$ the disc angular velocity, and $\sigma$ its orthogonal 1-D velocity dispersion.

The largest clumps reach radii of $1 \mathrm{kpc}$ (e.g., [325]) and masses of a few percent of the disc mass. Galaxies containing baryon mass of $10^{10}-10^{11} M_{\odot}$ typically form clumps of mass $\simeq 10^{8}-10^{9} M_{\odot}($ see $[320,322,323])$.

Those long lived clumps $\left(\simeq 2 \times 10^{8} \mathrm{Myr}\right)$ remain in Jeans equilibrium [322], and are rotationally supported. Ref. [325] showed that the clumps to survive when, in agreement with the Kennicutt-Schmidt law, the gas is converted into stars at a rate of a few percent, similarly to local star-forming systems. The gas clump remains bound, converting into stars, and thus migrates to the galaxy centre. During the collapse phase, baryons are compressed (adiabatic contraction, Refs. [270,271], e.g., at $z \simeq 5$ in the $10^{9} M_{\odot}$ galaxy shown in Figure 6), making the DM profile more cuspy. As dynamical friction (DF) between baryons and DM transfers energy and angular momentum to the DM component, the clumps migrate to the galactic centre. The cusp then heats up, and forms a core.

At later stages (e.g., around $z=2$ ), supernovae explosions provide other events where gas expulsion from the supernova explosion decreases the surrounding stellar density. However, as soon as the smallest clumps form stars with a small part of their mass, they get destroyed by such feedback 9 .

Despite some considering that SNFF and DFBC scenarios represent different implementation of the same idea, based on some common features (e.g., gravitational interaction yielding clumps to DM transfer of energy), they essentially differ for two main reasons: Firstly, the epoch of effective profile flattening are markedly different. While the flattening provided by the DFBC (see Figure 6), starts at higher redshifts $(z<5)$, at $z \simeq 3$ the DM density profile, in the pure SNFF scenario, the flattening remains similar to the NFW profile $[298,300,326]$.

Secondly, the energy sources moving the clumps come from opposite natures: in the SNFF scenario, the energy of supernovae is driving the clump [75,76], while the DFBC clumps just "passively" infall to the galactic centre, in the sense of Ref. [75,76]'s definition.

9 The process of star formation is not efficient. 
Refs. [327,328] compared the two scenarios with high resolution data, from Refs. [190,195], LITTLE THINGS [188], THINGS dwarfs [36,164], Sculptor, Fornax and the Milky Way, examining their respective predictions for the slope-stellar mass, and the slope-circular velocity relationships. They found the DFBC scenario to perform slightly better than the SNFF, in addition to predicting, in DFBC and differently from SNFF, the emergence of cores at smaller stellar masses than $10^{6} M_{\odot}$. However, even the DFBC mechanism cannot produce cores in very small dwarfs $\left(M_{*} \leq 10^{4} M_{\odot}\right)$ in agreement with Ref. [329] results.

Finally, recall that cusp can reform in galaxies with a bulge [171], We recall that in galaxies having a bulge, the cusp can reform as shown by [171], even in dwarf galaxies [308].

\subsection{Cosmological Solutions}

As previously discussed, the SSP $\Lambda$ CDM model could perfectly well hint at the CDM's paradigm failure. In such a case, the nature of dark matter should be changed, or that of gravity modified, in models, both of which have already been widely checked with various degrees of success.

The simplest possibility grants DM with a small velocity dispersion $(\sigma \simeq 100 \mathrm{~m} / \mathrm{s}$ nowadays, also denoted by a small DM relic mass) [230,330], and is usually referred to as warm dark matter (WDM). As past values velocity dispersion should be higher, such smear could affect small scale structures. This idea leads to an effect resembling the baryonic solutions discussed above: as DM particles retain higher velocity than in the CDM paradigm, small scales cluster less, leading both to flatter profiles and fewer low mass haloes. Many simulations checked WDM structure formation (e.g., [326,331-333]).

Although tuning the WDM particle mass to the scale of the halos considered can solve several of the CDM problems, it is not able to get the correct rotation curves for all galaxies or in the entire mass range for which CDM has problems [334]. Both pure CDM and WDM models were explicitly tested against disk galaxies observed rotation curves by Ref. [335], finding no match, however, taking baryons into account, hydrodynamical simulations find the correct rotation curves (see the discussion in Section 4 above).

Moreover, WDM produces too few subhaloes compared to, say strong-lensing subhalo fraction, as shown by the $m=2 \mathrm{keV}$ thermal relic of Ref. [331], and by several other authors (e.g., [336-338]). A $1 \mathrm{kpc}$ core requires a $0.1 \mathrm{keV}$ thermal candidate, while large scale structure imply $m \simeq 1-2 \mathrm{keV}$, corresponding to cores of 10-20 pc (see Figure 9, and Ref. [339]). WDM too sharp spectrum fall off lead [340] to conclude it does not improve on $\Lambda$ CDM. To make it worse, WDM alters the Lyman- $\alpha$ forest structure [341] and thus cannot consistently solve the small scale problems and the observed structure of the Lyman- $\alpha$ forest.

The next simple possibility endows DM with self-interaction (Self-interacting DM, hereafter SIDM, [236]), with cross-section of same order as for nucleon-nucleon $\left(\simeq(\mathrm{m} / \mathrm{g})^{-1} \mathrm{~cm}^{2}\right)^{10}$. Redistribution of angular momentum and energy results from elastic scattering in the galaxies inner region, reduces tri-axiality and forms a Burkert profile core [342]. Some cosmological simulations [212,343-345] running with $0.1-0.5 \mathrm{~cm}^{2} / \mathrm{g}$ cross sections, consistent with clusters of galaxy merging observations, [346-348], claim that this scattering mechanism solves the CC problem in dwarfs, MW sized galaxies, and clusters of galaxies. The result of [343]'s simulations are presented in Figure 10 for halo mass ranging from galaxies to clusters and two values of $\sigma / m$. As the cored SIDM subhaloes feel more tidal stripping and disruption than CDM subhaloes, it solves the CC problem and improves on WDM [343,345] since it leaves enough subhaloes (see however [334] for a different point of view). Note also that SIDM have some natural appeal since particle models of the "hidden sector" produce them, e.g. [236,349,350].

$101 \mathrm{~cm}^{2} / \mathrm{g} \simeq 1 \mathrm{barn} / \mathrm{GeV}$ 

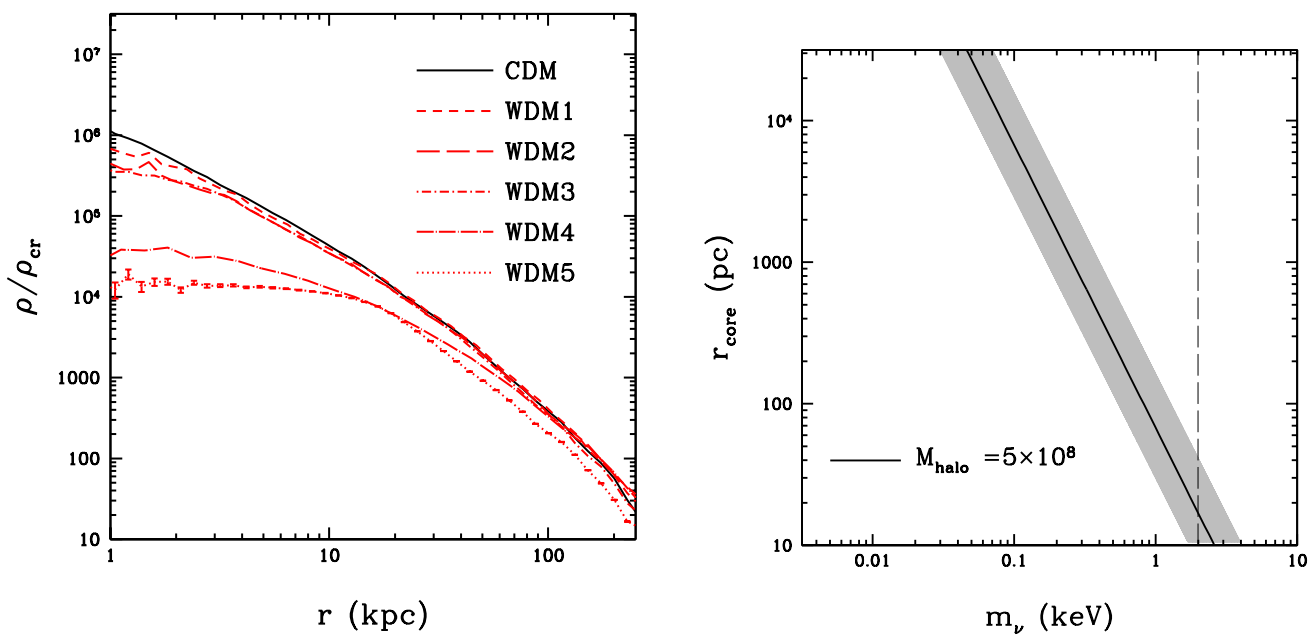

Figure 9. Left panel: comparison of a CDM density profile (solid black line) with five WDM models' profile ranging from $m=2 \mathrm{keV}$ (WDM1) to $0.05 \mathrm{keV}$ (WDM5). Right panel: core radius obtained in terms of the WDM particle mass. The shaded band corresponds to allowed cosmological values $0.15<\Omega_{m}<0.6$. The vertical line represents the upper limit WDM mass, constrained from large scale observations (reproduced from [339]).

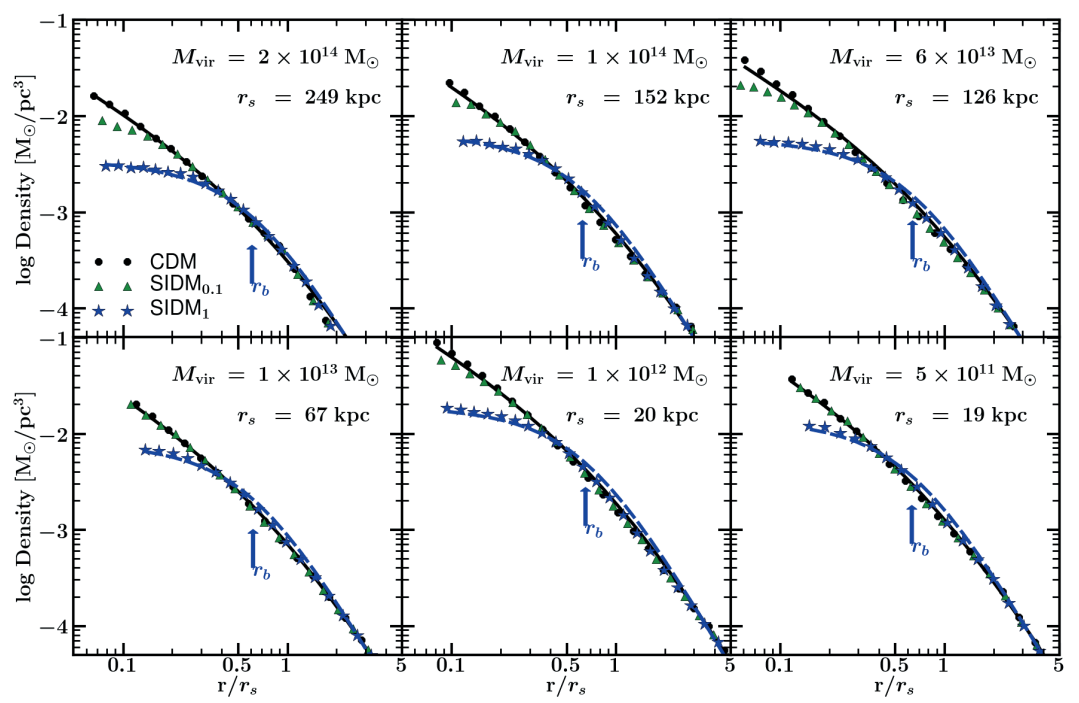

Figure 10. Comparison of the NFW (black line) and Burkert (blue line) density profiles with simulations of SIDM universes, using the two cross section over DM particle masses $\sigma / m=1$ and 0.1, denoted $S I D M_{1}$ (blue stars) and $S I D M_{0.1}$ (green triangles) respectively. The arrow indicated the location of the Burkert's profile core radius (figure reproduced from [343]).

SIDM has been declined in several variations with altered properties: negative scattering leads to repulsive DM (RDM) [232], a condensate of massive bosons, which superfluid behaviour in the central part of DM haloes smoothes down the cuspy profiles [351]. Such non-relativistic Bose condensate was recently simulated for structure formation [352], where large scale structures could not distinguish CDM universes from their model, while flat density profiles and reduced small scale substructures resulted from the opposition, at small scales, between gravity and the uncertainty principle. A further implementation of this model using scalar field condensation, dubbed Scalar Field Dark Matter (SFDM), produced galaxies flat inner profiles [353]. 
Wave-particle duality was summoned for Fuzzy DM (FDM) [231], ultra-light ( $m \simeq 10^{-22} \mathrm{eV}$ ) scalar particles which galactic core sized Compton wavelength cannot be "squeezed" further, resulting in flatter profiles and less substructure.

The last two aspects of SIDM are very common when related to indirect DM detection: Self-Annihilating DM (SADM) [234] proposes that the self-interaction results in annihilation, with cross section-velocity $\sigma v \simeq 10^{-29}(\mathrm{~m} / \mathrm{GeV}) \mathrm{cm}^{2}$. In dense regions, the annihilation leads to possibly detectable levels of radiation. Annihilation reduces the structure's particle number in the centre, thus reducing gravity and consequently expanding particles orbits and flattening the density profile.

The second aspect, Decaying DM (DDM) [235], considers DM to decay into relativistic particles, also leading to radiation detection. The gravitational effect is similar to SADM in structure, reducing significantly the core's density of galaxies while large scale structures remain unaltered.

\subsection{Modified Theories of Gravity}

The preceding alterations of DM spoil the simplicity of the CDM paradigm, a further possible solution to the small scale problems legitimately questions the existence of DM and proposes changing gravity itself: this leads to the branch of modified theories of gravity (MG). Although MG is an old issue, the discovery of the universe's accelerated expansion [354,355] literally exploded interest in it. Most of the more recent alternatives to GR are cosmologically motivated, attempting to replace or supplement the concordance cosmology postulates of "inflation", "dark matter" and "dark energy" [356]. They all build on the premises that, although agreeing with GR locally in time and space, gravity may be quite different in the early universe or at large scales.

In this context, instead of interpreting the "anomalous" rotation curves of spiral galaxies nowadays as the trace of missing mass (DM), they reveal a lack in the gravity theory.

From the discovery of the universe's accelerated expansion by the supernova surveys, Einstein's cosmological constant was rapidly reinstated, and quintessence was proposed to overcome the problems that such constant entails. Alternatives to GR also attempted to explain such results.

Nowadays, the catalogue of MGs theories, cosmologically motivated or not, extends to: $f(R)$, $f(T)$, Modified Newtonian Dynamics (MOND), BIMOND, Tensor-Vector-scalar theory (TeVeS), Scalar-Tensor-Vector Gravity Theory (STVG), Gauss-Bonnet MG, Lovelock MG, non-minimal scalar coupling, non-minimal derivative coupling, Galileons, Hordenski, etc. (for a review, see [357] and Figure 11, reproduced from their paper).

MOND demonstrated particular effectiveness in solving the SSP $\Lambda$ CDM. An originally ad hoc modification of Newton's gravitation law was proposed in 1983 by Milgrom [244,245], for Newton's second law ${ }^{11}$ :

$$
F=m \mu\left(a / a_{0}\right) a
$$

There, with the universal constant $a_{0} \simeq 10^{-10} \mathrm{~m} / \mathrm{s}^{2}$, the gravitational force results nonlinearly in the acceleration, $a$, as the functional form $\mu\left(a / a_{0}\right)$ tends to 1 for high values of the acceleration, while small accelerations modify Newton's law with $\mu \sim a / a_{0}$.

The analytical form of $\mu(a / a 0)$ in MOND remains free to be determined from observations. RCs-fitting leads to $\mu(x)=x / \operatorname{sqrt}\left(1+x^{2}\right)$, while the so called interpolation function reads $\mu(x)=x /(1+x)$.

In both forms, $a>>a_{0}$ recovers Newton's second law, while the case $a<<a_{0}$ yields a force $F$ proportional to the velocity squared:

$$
F=m\left(a / a_{0}\right) a=m a^{2} / a_{0}=G m M / r^{2},
$$

11 However, this change in Newton's law violates momentum conservation 
such that

$$
a=\sqrt{G M a_{0}} / r
$$

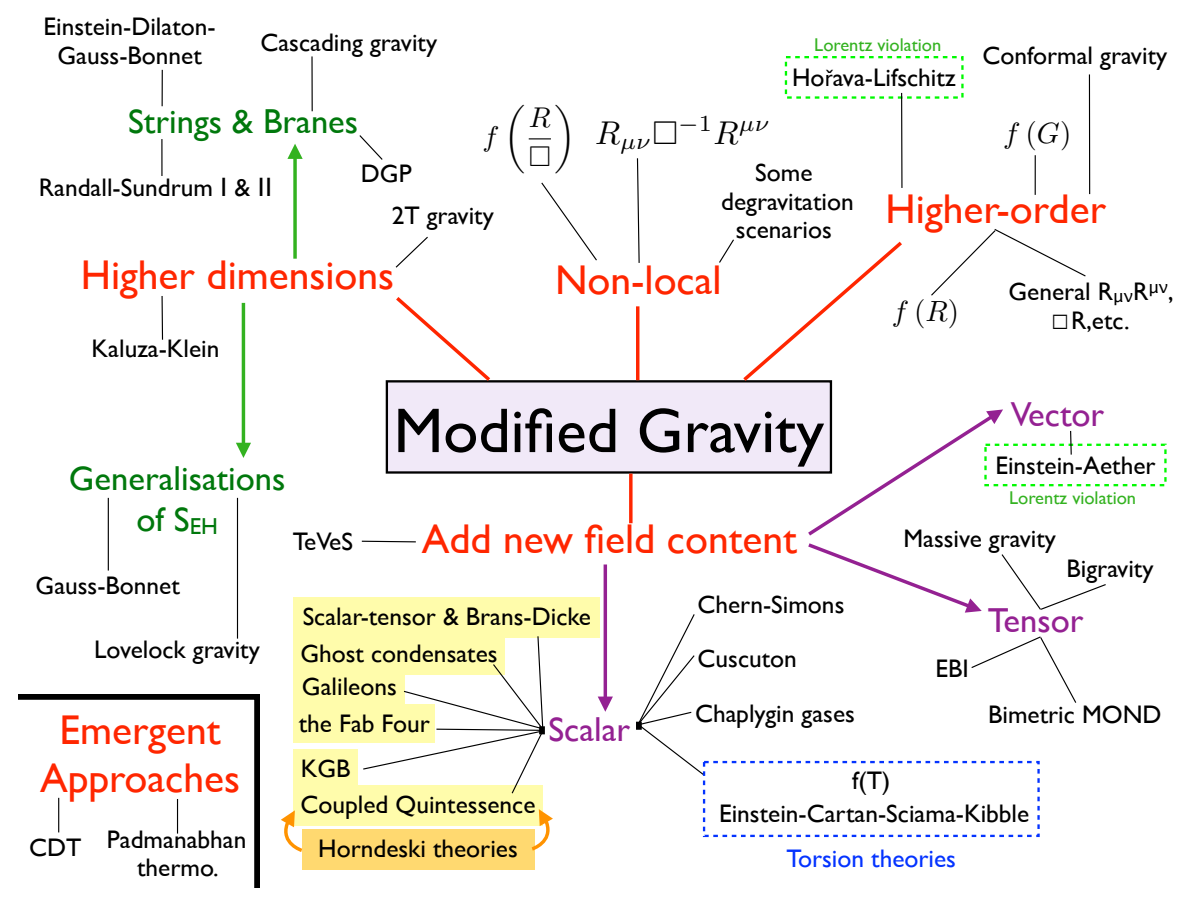

Figure 11. MGs tree diagram, reproduced from [358] (with permission from Tessa Baker).

For a test particle in circular motion around the galactic centre, for small acceleration, far away from the centre,

$$
a=v^{2} / r=\sqrt{G M a_{0}} / r,
$$

the rotation velocity reaches a plateau $V_{f}=\sqrt[4]{G M a_{0}}$ and one re-obtains the baryonic Tully-Fisher relation $V_{f}^{4}=G a_{0} M_{b}$.

The success of MOND extends beyond fitting the RCs of spirals, and reproducing the Tully-Fisher relation: it provides explanations for several galactic phenomena, from dwarfs to ellipticals (see [359,360]), to Freeman's law [361], that sets the upper limit for spirals surface brightness, to Fish's law [362], determining ellipticals characteristic surface brightness, and to the Faber-Jackson relation. MOND is actually the simplest way to reproduce observed scaling relations, such as the relation between the rotation curve's shape and the baryonic surface density (see Figure 15 of Ref. [359], relevant to the diversity of rotation curve shapes at a given $V_{\max }$ scale), with the stellar and dynamical surface densities in the central regions of disk galaxies (recently discussed in $[363,364]$ ), or the small scatter of the BTFR discussed in Ref. [122]. These tight relations appear as less natural consequences of baryonic solutions to the CC problem than of MOND, from a formal point of view.

The RCs of two different dwarf galaxies, UGC11583, and ESO138-G014 are plotted in Figure 12, together with their MOND fit. Although MOND usually fits galactic RCs well, some cases, such as ESO138-G014 here, escape its grasp. This should be taken with caution as, according to Ref. [365], there are misunderstandings on MOND on the problems they quote in their section 3. See also Ref. [366] for a different point of view.

At cluster scales, nonetheless, MOND proves much less successful (however, see [359] for a different point of view). In addition, MOND historically being a mere Newtonian fit, to be considered a full theory requires, also to be applicable on cosmological scales, a relativistic version. One such TeVeS theory was proposed by Sanders and Bekenstein [367-369]. 

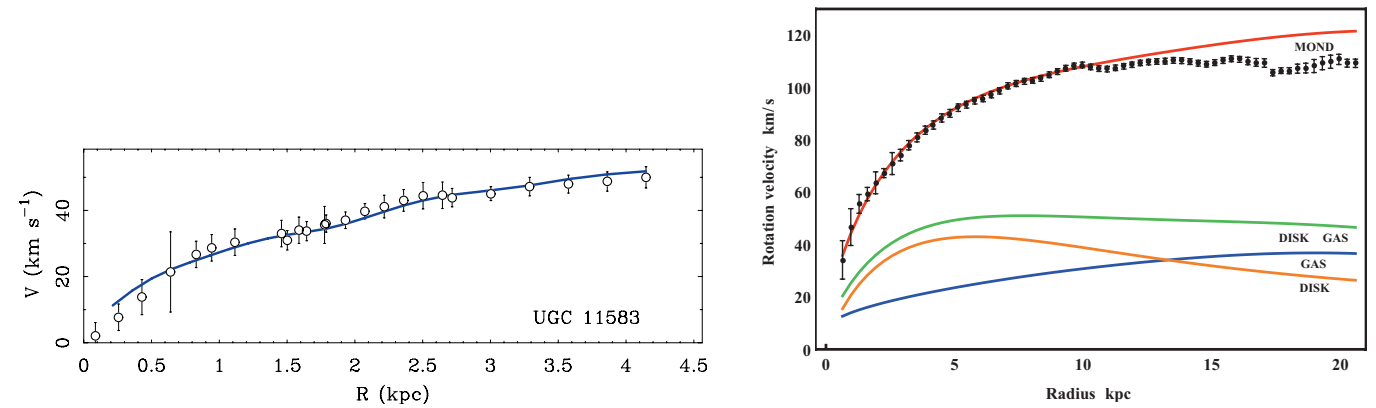

Figure 12. Left panel: Example of MOND fit (blue lines) to a dwarf galaxy (UGC11583, reproduced from [359], Figure 25). Right panel: RC comparison between observed ESO138-G014 (black dots with error-bars) and MOND prediction (red line) (reproduced from [370]).

Since the successes and problems of MOND and the $\Lambda$ CDM model appear on complementary scales (galactic vs large scales), Khouri [371] proposed combining both theories, keeping each on their respective successful scales. A wide survey of MOND's successes and problems, and of its attempted relativistic extensions was presented in [359].

In summary, there exist several apparently valid proposals solving the CC problem, as well as for the other small scale problems, but the challenge is to single out the correct one.

\section{The Missing Satellite Problem}

Galactic mass halos were noticed by Klypin et al. and Moore et al. [32,41] to present many more subhaloes predicted by N-body simulations than observed satellite galaxies. The scale invariant CDM primordial fluctuations at small scales leads, through collapse, to a large number of subhaloes, hence creating this MSP.

The MW counts 9 bright dSphs, Sagittarius, the LMC and the SMC, thus much less than the 500 satellites, obtained in simulations, with larger circular velocities than Draco and Ursa-Minor (i.e., bound masses $>10^{8} M_{\odot}$ and tidally limited sizes $>$ kpc, see Refs. [34,35] and Figure 13, top left panel).

The problem was confirmed in subsequent cosmological simulations (Aquarius, Via Lactea, and GHALO simulations: $[158,372,373])$. In the end, every cosmological simulations predicts Milky Way-like galaxies surrounded with at least one order of magnitude more small subhalos (dwarf galaxies) than observed (e.g. Via Lactea simulation [373]).

The ultra faint dwarf satellites discovery (UFDs) [374-378] alleviated the problem without solving it: adding them to known MW satellites reduces the discrepancy (see surveys like SkyMapper, DES, PanSTARSS, and LSST [379]).

The key idea of the solution lies in the distinction between visible satellites and the entire population: if only a subset of the population is visible, the observed vs predicted satellites discrepancy can be reduced. Thus, various suppression mechanisms for the visible population have been proposed:

a. Tidal stripping from the satellites' parent: presently observed satellites had the largest masses before accretion (LBA), large enough to retain visible stars, resisting tides when accreted by their parent [373].

b. Re-ionisation stripping satellites gas, thus star formation, hence suppressing visible satellites formation [380,381]: presently observed, earliest forming satellites (EF see Refs. [32,380] and Figure 13, top right, and bottom left) are visible because they acquired gas, and thus form stars, before re-ionisation.

Ref. [382] compared the cumulative number of dSphs and UFDs (with $M / L \simeq 1000$, from SDSS data) with the cumulative number of satellites obtained in the Via Lactea simulation, assuming a $z=9-14$ reionisation epoch, almost solving the problem (see Figure 13, bottom right panel). 
c. Photo-ionisation from stellar and supernova feedback (e.g., Refs. [310,383,384]), and generally stripping gas by ram pressure (e.g., Ref. [385]). Because of the photo-ionisation threshold, UFDs' baryons to stars conversion efficiency lies in the range $0.1 \%-1 \%$, thus making it is not clear whether they are "fossils" from reionisation epoch [386].

d. Transfer of angular momentum from baryons to DM through dynamical friction [166,273], which turns also cuspy profiles to cores. The number of visible satellites decreases since tidal stripping acts more on cored density profiles.
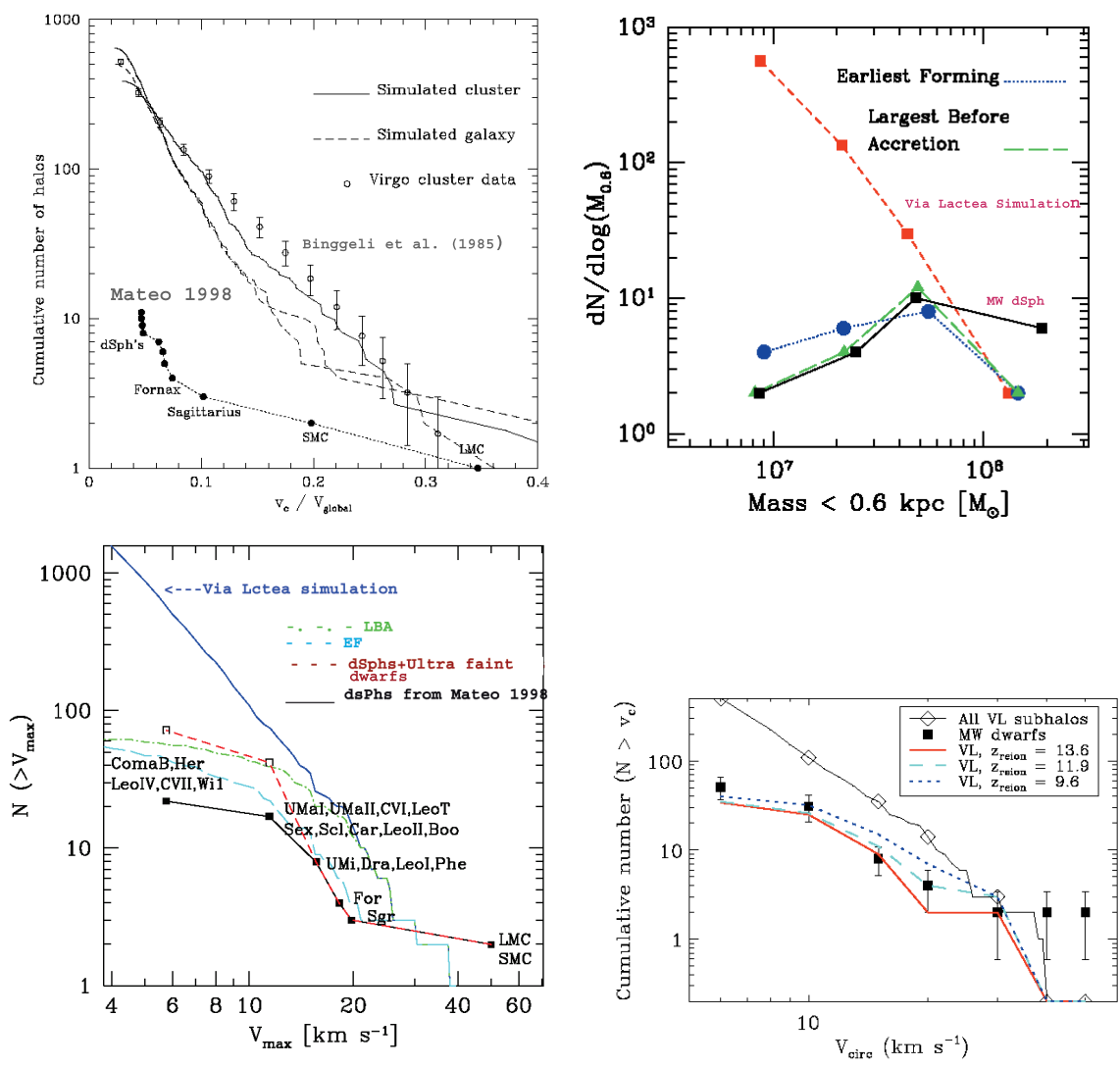

Figure 13. Top left panel: comparison, (from [32]) of the simulated cumulative clusters and dSphs number with observed clusters [387] and dSphs [388]. Top right panel: comparison of the abundances for the Via Lactea simulation (red dashed line) with the MW dSphs in the EF (blue dotted line), and LBA (green dashed line) scenarios (from [389]). Bottom left panel: comparison of the abundances for the Via Lactea simulation (blue solid line) with the MW dSphs (black line) , MW dSphs+Ultra-faint dwarfs (brown dashed line), and the EF (blue dashed line), and LBA (dot-dashed green line) scenarii (from [390]). Bottom right panel: comparison of the abundances for the Via Lactea Simulation (black solid line) with the MW dSphs+Ultra-faint dwarfs (black dots), and with the Via Lactea subhaloes at redshifts 9.6 (dashed dark blue line), 11.9 (dashed light blue line), and 13.6 (solid red line), respectively (from [382]).

In conclusion, adding baryon physics to the usual dissipationless DM model solves the MSP, for the majority of the Cosmology community, as well as the other SSP $\Lambda$ CDM, as shown by recent hydrodynamic simulations (e.g., [73,119,391]) or semi-analytic models [59].

\section{The Too Big to Fail Problem}

On closer inspection, eliminating visible satellites from the faint end of the distribution does not exhaust the model vs observation discrepancies in satellites (MSP): the most massive (luminous) satellites also pose problem. Boylan-Kolchin [34,35] discovered, in the Aquarius and the Via Lactea 
simulations, a population of $\simeq 10$ subhaloes that were too massive and dense, by a factor $\simeq 5$, to host even the brightest satellites of the MW, and dubbed it the "Too Big to Fail" (TBTF) problem ${ }^{12}$. In general, $\Lambda \mathrm{CDM}$ simulations of the MW predict at least 10 subhaloes with $V_{\max }>25 \mathrm{~km} / \mathrm{s}$, while $12<V_{\max }<25 \mathrm{~km} / \mathrm{s}$ for all the dSphs.

This is shown in Figure 14. Both the left, and right panels display RCs with $V_{\max }<24 \mathrm{~km} / \mathrm{s}$ while simulations have much larger values. Rotation curves obtained from NFW-shaped subhaloes with $V_{\max }=(12,18,24,40) \mathrm{km} / \mathrm{s}$, with a $1 \sigma$ scatter, taken from Aquarius simulations, present the simulation side of the TBTF problem in the left panel. They are confronted, for this purpose, with the bright dSphs, the dots with error-bars. The results show that $V_{\max }<18 \mathrm{~km} / \mathrm{s}$ for most of the $\mathrm{dSphs}$, all have $V_{\max }$ under $24 \mathrm{~km} / \mathrm{s}$ and only Draco is consistent with a subhalo modelled with $V_{\max } \simeq 40 \mathrm{~km} / \mathrm{s}$. The right panel plots $V_{\max }$ vs the visual magnitude, $M_{V}$, and confirms that the simulations produce much more massive subhaloes than the observed dSphs.
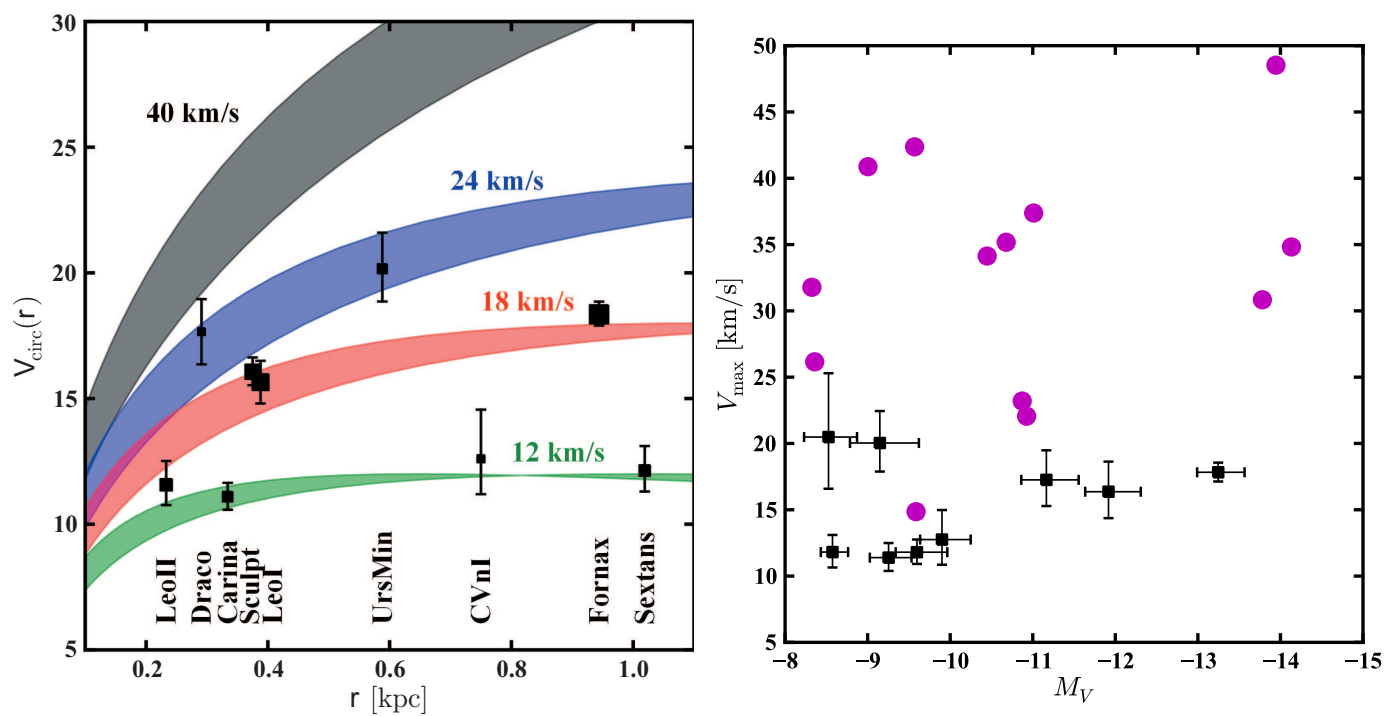

Figure 14. Left panel: rotation curves for NFW subhaloes with $V_{\max }=(12,18,24,40) \mathrm{km} / \mathrm{s} \pm 1 \sigma$ from Aquarius simulations, confronted with the bright dSphs, represented by the dots with error-bars. Right panel: $V_{\max }$ vs. $M_{V}$, the visual magnitude, for the same bright dSphs (black dots with error-bars) and the most massive subhaloes of the Aquarius simulations (magenta dots). The observed dSphs are much less massive than the simulations (panels reproduced from [35]).

This issue can be compared with the CC problem for haloes: the $\Lambda$ CDM seems to produce too much mass in haloes and subhaloes. Thus, similarly to the CC problem, two main classes of solutions have been proposed: cosmological and astrophysical. As for the CC problem, cosmological solutions modify the perturbation spectrum or the nature of dark matter particles. Astrophysical solutions are driven by baryon physics, and consider, in analogy with solutions to the CC problem:

a. The shape of satellites inner densities, shifting from cuspy to cored [73,74] (hereafter Z12 and B13 respectively), thus making them more susceptible to tidal stripping and even to tidal destruction $[77,389]$. This picture would see the present-day dwarf galaxies, more massive in the past, transformed and reduced strong tidal stripping (e.g., [392]);

b. The suppression of star formation from

(a) Supernova feedback (SF)

12 "Too Big to Fail" refers to simulation satellites being too big for MW satellites, while no mechanism would lead them to fail being visible. 
(b) Photo-ionisation [74,393], and

(c) Reionisation. This can prevent small mass DM haloes gas accretion, "quenching" star formation after $z \simeq 10[380,381,394]$.

This would suppress dwarfs formation or could make them invisible;

c. The dynamical effects of a baryonic disc [73,74]. Satellites crossing such disc experience disk shocking, strong tidal effects, even more so for cored inner profiles.

Alternative solutions to the TBTF problem have been proposed: the TBTF excess of massive subhalos in simulations on the MW could vanish if Einasto's are the correct satellite density profiles, or if the correct measurement of MW's virial mass reduces from $\simeq 10^{12} M_{\odot}$ to $\simeq 8 \times 10^{11} M_{\odot}[395,396]$.

The above solutions to the TBTF problem are focused on the MW satellites. However, the TBTF problem also concerns the Local Group, and Local Volume galaxies [305,306,397], galaxies that are too massive to be modified by (reionisation) feedback [35,72,291,301,398-400], contrary to the baryonic solution of Refs. [59,73,74,401]. Nevertheless, such limitations of feedback were challenged for a few galaxies by $[137,302,314]$, and for an entire galaxy population by [402].

\section{A Unified Baryonic Solution to the $\Lambda$ CDM Small Scale Problems}

So far, each SSP $\Lambda$ CDM was solved separately through different recipes. Unified solutions were however proposed, after understanding their inter-relations. For example, transforming cuspy density profiles into cored distributions, through the SNFF or the DFBC scenario, also affects the parent halo distribution and number of substructure/satellites as tidal effects of a parent halo on a satellite depend fundamentally on its shape (e.g., [75-77]): cuspy satellite structure will not suffer big changes when entering the main halo, while the parent's tidal field can easily strip a cored profile from its gas, in some cases down to its destruction [77].

A recent sketch of a baryonic solution to the SSP $\Lambda$ CDM was proposed [73,74], based on SF explosions $[58,75,76,278-280]$ removing angular momentum and gas from the proto-galaxy, thus flattening the density profile. Indeed, the discrepancy between observed and predicted number, and density, of satellites (see [74]) can significantly reduce, by applying a correction to satellites circular velocity at $1 \mathrm{kpc}, v_{1 \mathrm{kpc}}$ [73], to large N-body simulations results (e.g., Via Lactea II, VL2 hereafter).

A similar result is obtained in the DFBC $[166,247,248]$. Indeed, the Del Popolo model [166] provided a similar correction to $v_{1 \mathrm{kpc}}$ than Zolotov et al. [73], which, applied to the Via Lactea satellites, as in Brooks et al. [74], simultaneously solves the MSP and the TBTF problems [59,403]. The model of Ref. [403] is in fact more complete: it couples satellites interaction with the halo to dynamical friction energy and angular momentum exchange from baryons clumps to DM, which flattens the density profiles. The effects of tidal stripping and heating on the satellites were modelled with a combination of the procedures from Refs. [77,404].

In summary, the method proceeded in two main phases. The first phase calculated the satellite density profile flattening from baryonic physics (in particular, the subhaloes' central mass lowering), considering it isolated, without interactions with the host halo, in the same fashion as in Ref. [166] (see also [405,406], to have a semi-analytical description of halos growth).

The flattening was translated into the difference in circular velocity, at $1 \mathrm{kpc}$, between the DM-only (hereafter DMO) satellites equivalent to those considered and containing also baryons (hereafter DMB satellites), $\Delta v_{\mathrm{c}, 1 \mathrm{kpc}}=v_{\mathrm{c}, \mathrm{DMO}}-v_{\mathrm{c}, \mathrm{DMB}}$.

Figure 15 displays the resulting $\Delta v_{\mathrm{c}, 1 \mathrm{kpc}}$ at $z=0$ and the fitted dashed line through the output points of the model, given by

$$
\Delta v_{\mathrm{c}, 1 \mathrm{kpc}}=0.3 \mathrm{v}_{\text {infall }}-0.3 \mathrm{~km} / \mathrm{s} \quad 10 \mathrm{~km} / \mathrm{s}<\mathrm{v}_{\text {infall }}<50 \mathrm{~km} / \mathrm{s}
$$

The second phase no longer considered the satellite as isolated, and subjected it to the host halo's tidal field and accretion. 


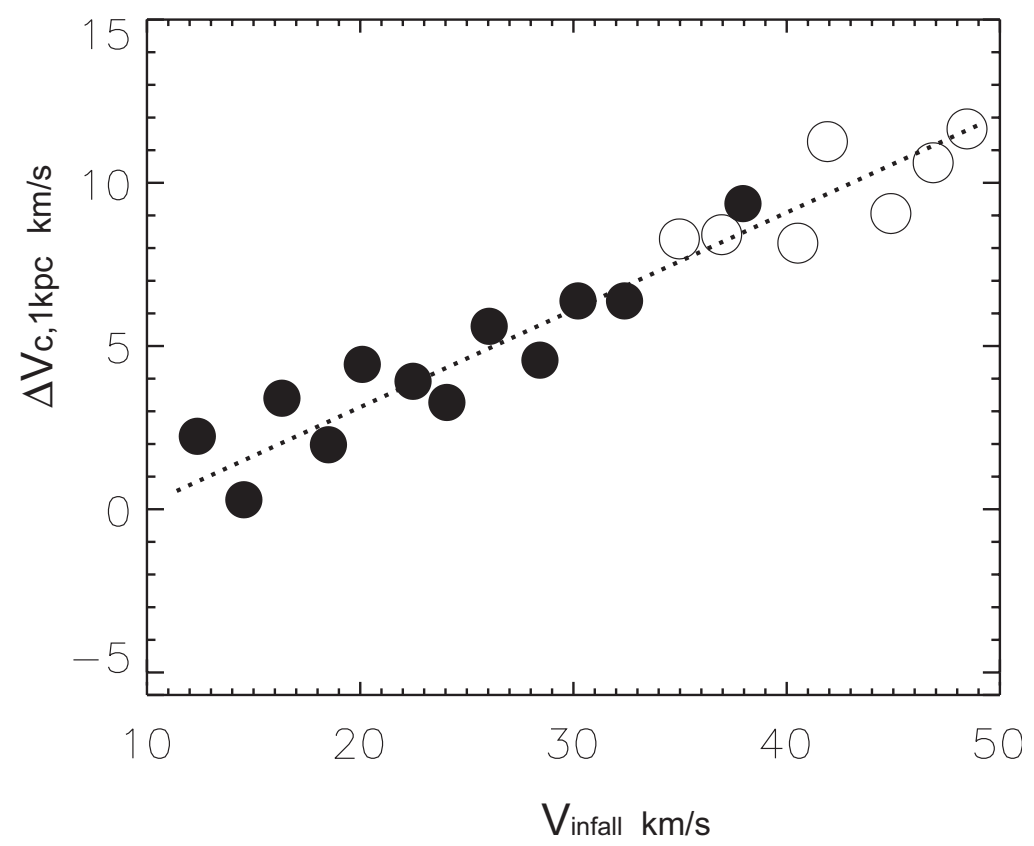

Figure 15. Flattening of satellites from inner Baryonic tidal stripping and heating, expressed in terms of difference in central $v_{\mathrm{c}}$ at $1 \mathrm{kpc}$, and $z=0$, between equivalent $\mathrm{DMO}$, and DMB satellites as a function of equivalent DMO satellites maximum velocity $v_{\max }$ at infall. The model produces, for satellites with $M_{b} / M_{500}<0.01$, filled circles, while for $M_{b} / M_{500}>0.01$, the open circles and results in a linear fit (dashed line) (figure reproduced from [403]).

The merger history and interacting satellites' growth was followed, and the substructure evolution was tracked in Ref. [403]'s model, taking into account the mass loss induced from tidal stripping, tidal heating, as well as the disc's stripping enhancement, caused by the host halo.

The destruction rates by tidal stripping induced a second correction to satellites outputted from $\mathrm{N}$-body simulations, requiring the link between satellite mass loss, or remaining, and velocity change (e.g., $V_{\max }$ ) during infall in the parent. Such link was shown in Figure 16: DMB satellites were shown to lose more mass than DMOs because

a. DMB satellites contain gas, DMOs do not;

b. $\quad$ DMB satellites profiles are flatter than DMO's, thus tidal stripping affects them more (e.g., [77]). Similar trend affect baryon-richer DMB (filled circles) compared to baryon-poorer DMB (open circles).

The maximum tidal loss for DMB satellites corresponds to those in the host galaxy disc's vicinity.

The analytic fits from Equation (8) of [77], linking the $v_{\max }$ change to tidal stripping mass loss,

$$
\frac{v_{\max }(z=0)}{v_{\text {infall }}}=\frac{2^{\zeta} x^{\eta}}{(1+x)^{\zeta}}
$$

where $x \equiv \operatorname{mass}(z=0) / \operatorname{mass}(z=\operatorname{infall})$, was also shown in Figure 16, for the exponent values $\zeta=0.40$ and $\eta=0.24$, corresponding to central density profile logarithmic slopes $\gamma=1.5$, represented by a dashed line, $\zeta=0.40$ and $\eta=0.30$, corresponding to $\gamma=1$, with a solid line, and $\zeta=0.40$ and $\eta=0.37$, yielding $\gamma=0$, with a dotted line, respectively. 


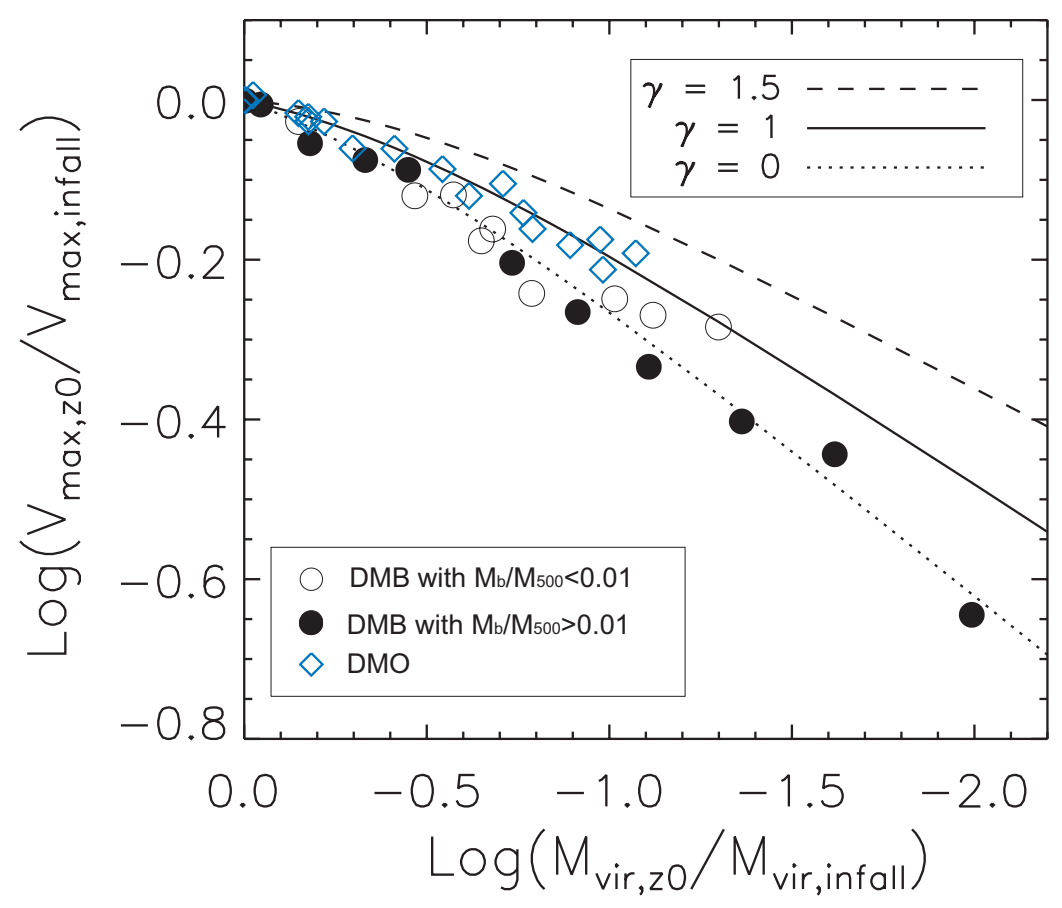

Figure 16. Reduction of the $1 \mathrm{kpc}$ circular velocity after infall, from $z_{\text {infall }}$ to $z=0$, in terms of corresponding mass reduction. DMB satellites with baryonic fraction $M_{b} / M_{500}>0.01$ are shown as filled circles, while $M_{b} / M_{500}<0.01$ yield open circles and DMO satellites are marked as open diamonds. Equation (8) of [77] fit for slope $\gamma=1.5,1,0$ are displayed as dashed, solid, and dotted lines, respectively (figure reproduced from [403]).

Then following [74], a destruction criterion (in terms of mass loss) was fixed, determining tidally disrupted satellites from Equation (6): mass losses

- $\quad$ above $97 \%$ mass $(x=0.03)$, or

- above $90 \%$ mass, with $v_{\text {infall }}>30 \mathrm{~km} / \mathrm{s}$ and pericentric distance $<20 \mathrm{kpc}$,

for a given satellite, was set as destroyed. The photo-heating induced star formation suppression, from the [393] results, produced a third correction. The last step assigned surviving satellites luminosity. Stellar masses of satellites were allocated relating their infall velocity $v_{\text {infall }}$ to the stellar mass $M_{*}$, as plotted in Figure 17. The fit to the $v_{\text {infall }}-M_{*}$ data yielded the relation ${ }^{13}$

$$
\frac{M_{*}}{M_{\odot}}=0.1\left(\frac{v_{\text {infall }}}{\mathrm{kms}^{-1}}\right)^{5.5} .
$$

Finally, the V-band magnitude, $M_{\mathrm{V}}$ was related to $M_{*}$ by applying [74]'s relation from [73]'s simulations,

$$
\log _{10}\left(\frac{M_{*}}{M_{\odot}}\right)=2.37-0.38 M_{\mathrm{V}}
$$

13 Note that scatter in the $v_{\text {infall }}-M_{*}$ relation proceeds from a halo mass reduction caused by tidal stripping and heating from $z_{\text {infall }}$ to $z=0$. 


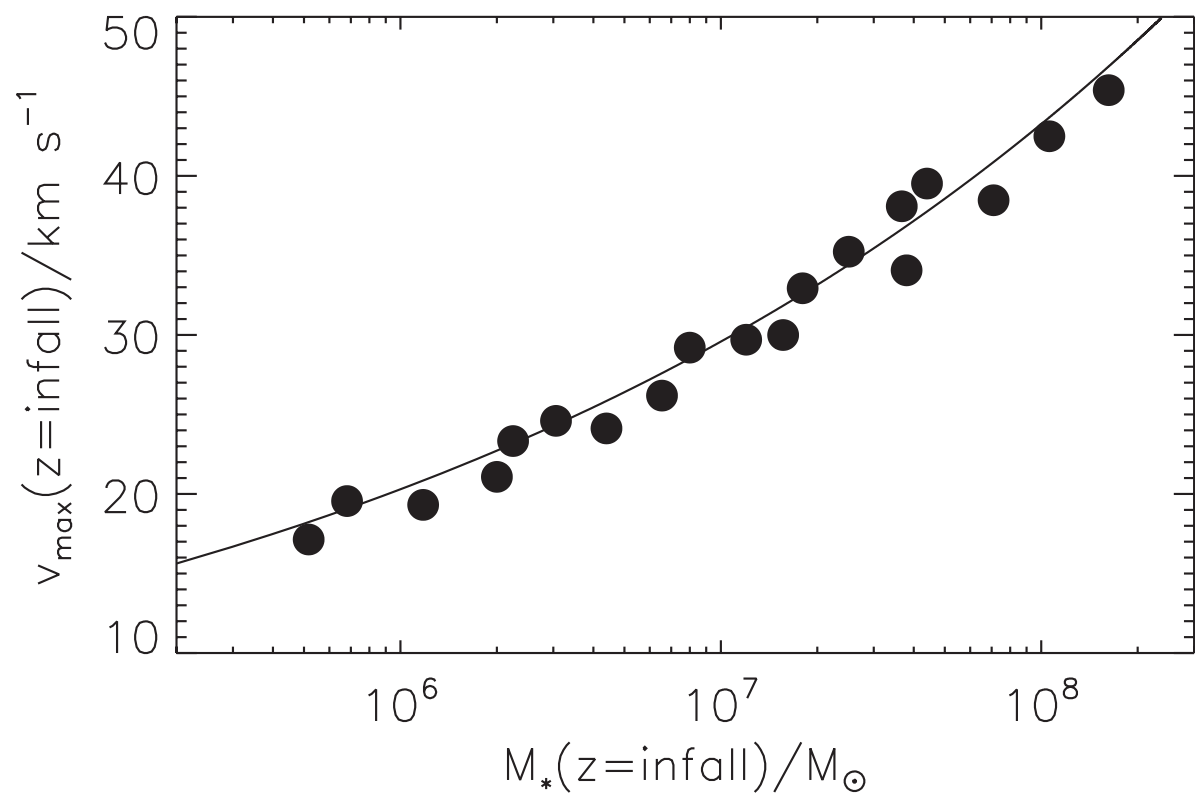

Figure 17. DMO subhaloes maximum velocity $v_{\max }$ as function of their stellar mass, $M_{*}$, at infall time. The stellar mass change with $v_{\text {infall }}$ is synthesised by the data fit from Equation (7), shown as a solid line (figure reproduced from [403]).

Figure 18 showed the result of the all corrections, with the raw results from the VL2 simulations at $z=0$ displayed on the top panel, to compare with the same satellites corrected with heating, destruction, and velocity corrections, as discussed above, presented on the bottom panel. There,

- Red filled symbols mark "observable" objects produced by the VL2 simulation,

- Filled black circles mark much fainter satellites than "observable", stripped of their stars (see $[74,77]$ ), their mass loss $\geq 90 \%$ still does not grant them destruction from the above criteria,

- Empty circles indicate totally dark objects

- Simple empty circles merely lost all their baryons and thus did not form stars as their mass was smaller than the minimum to retain them, while

- Empty circles crossed with an " $x$ " represent destroyed subhaloes from baryonic effects (e.g., baryonic disc, etc).

In agreement with [74], 3 satellites with $v_{1 k p c}>20 \mathrm{~km} / \mathrm{s}$ were obtained.

On top of a reduction of the number of satellites to reach the levels observed in the MW, noticeable from Figure 18, clearing up the MSP, the model, applying baryonic correction to subhaloes, also reduces their central velocity, solving the TBTF problem. As evidenced in Figure 19, those corrections from baryonic physics are sufficient to solve the velocity and number counts problems of MW-type satellites produced by the VL2 simulation. 


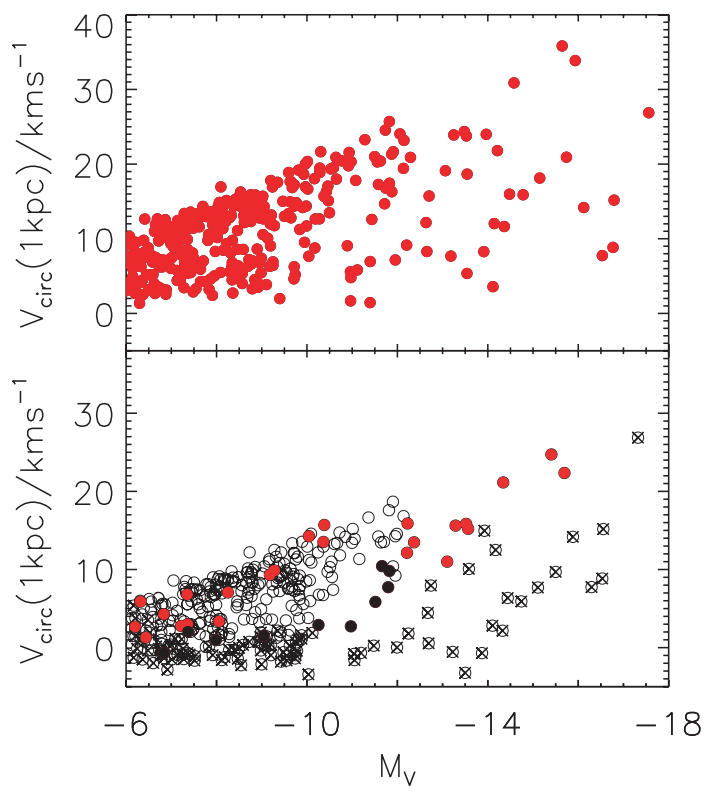

Figure 18. Baryonic effects on VL2 simulation subhaloes, seen through their changes in $v_{1 k p c}$ vs. $M_{V}$. VL2 satellites are labelled, as in [74], in the top panel, by their velocities vs. $M_{V}$ at $z=0$. The same, after baryonic corrections, are presented in the bottom panel. Satellites stripped of their stars from losing enough mass are represented by filled black circles: at infall, their actual luminosities are much fainter and indicated luminosities are only upper limits. Satellites actually observable at $z=0$ appear as filled red circles. Empty circles mark dark subhaloes, those with an $\mathrm{x}$ being most probably tidally destroyed (figure reproduced from [403]).

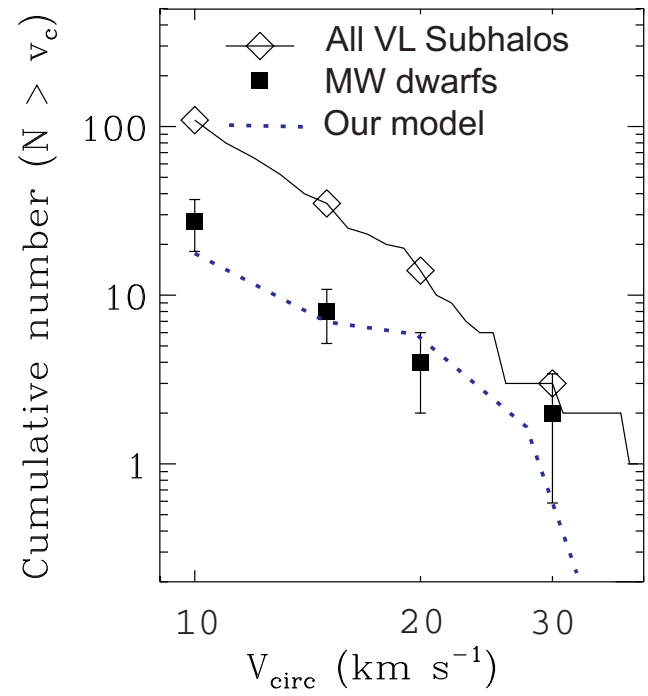

Figure 19. Corrections from baryonic physics to cumulative number of MW satellites in terms of circular velocity in VL2 simulation. The classical MW satellites augmented by the ultra-faint-dwarfs from [382] are shown as filled squares with error bars. The Via Lactea subhaloes abundance [373] produces the solid line with diamonds. The model of baryonic corrections applied to VL2 subhaloes yields the abundance shown by the dotted line (figure reproduced from [403]). 


\section{Conclusions}

The discussions on vertical motions of stars near the Galactic plane by Öpik [407] were the first evaluation of our neighbourhood's matter content. One century has passed since, and present evidence points towards a large part of matter in the universe consisting of DM. Despite this mounting evidence, direct or indirect experiments have still not detected DM particles, to date [4,5].

As DM dominated structures, dwarf galaxies, small scale structures, and galaxy satellites are at the front of our understanding of the nature of DM. Moreover, observation of those objects have presented significant discrepancies with predictions of the $\Lambda \mathrm{CDM}$ model. This review discussed some of those discrepancies such as the CC problem, and issues with satellites.

The understanding of the nature of dark matter, and/or the physics of galaxy formation certainly can improve a great deal from the study of those discrepancies. Noticed more than twenty years ago, we still have no definitive idea of what causes the CC problem. Recently, baryonic effect on small scale structure problems have been the focus of several researches. Interaction of baryon clumps with DM, from $M>10^{5} M_{\odot}$ dwarfs, in the DFBC scenario, can create cores, and MW-type galaxies acquire cuspy profiles, as shown in [327]. The SNFF scenario had found similar results, but with cores formed from heavier satellites, $M_{*}>10^{6} M_{\odot}[165]$. Both scenarios also solve the subhaloes abundance and TBTF problems by reducing the galaxies central DM. However, the baryonic effects debates are still open, as the SNFF scenario presents difficulties in some small scale issues, in particular in some of the MW classical dwarfs [291,301], while isolated galaxies do not clearly present baryonic physics solutions to the TBTF problem. In particular, the SNFF scenario, with future flat inner profiles dwarfs with $M_{*}<10^{6} M_{\odot}$, would conclude that DM is not cold ${ }^{14}$. Further investigations of the SIDM model or its variant would in such case be required to understand if they can solve the small scale issues. In any case, new hints on the small scale issues would require the future surveys to discover new satellites with their stars velocity measurements.

Be that as it may, the difficulty to distinguish cusps from cores retains the debate on dSphs structure open, as discussed in the introduction (e.g., [408]). GAIA [409] and the Subaru Hyper-Supreme-Camera [410] were proposed to provide possible solutions, but both instruments can only hope to solve the problems for larger dwarves, such as Sagittarius [411]. The stars velocity line of sight components and stars' $2 \mathrm{D}$ projection radius that they could provide are plagued with degeneracy between the anisotropy parameter and the density profile, because the determination of the latter is based on Jeans equations. Improvements were proposed measuring just one of three velocity components, and two of three position components [see 412], to obtain proper motions of the dwarves stars [389], and thus the half-light radius density slope, despite the challenge it poses with GAIA [411]. The knowledge on dwarves inner structures remains of fundamental importance, no matter the technical problems. Yet, the SNFF scenario's tension with the $\Lambda$ CDM model is not fundamental: as shown, the DFBC scenario provides a way to form cores from cusps before gas forms stars, and is more efficient. Thus, although the SNFF would have serious problems if cored dwarves with $M_{*}<10^{6} M_{\odot}$ were found, so long as $M_{*}>10^{5} M_{\odot}$, the DFBC scenario within the $\Lambda$ CDM model would not disagree with observations.

The Baryonic Tully-Fisher Relation and, mostly, the satellite planes problem remain open problems for the $\Lambda$ CDM model to tackle, although the first one seems to be on the right path.

Alternate tests of the $\Lambda \mathrm{CDM}$ could proceed from checking

- The number of small subhaloes in galaxies' virial radius,predicted to be large: gravitational lenses flux anomalies barely found agreement with those predictions [413];

- The Galaxy's cold tidal streams perturbations by subhaloes (see [414]).

14 This conclusion is not true in the DFBC scenario. 
Obviously, collider, direct, and indirect DM particles detection would solve the problem of DM nature and existence. Unfortunately, no evidence of super-symmetry (SUSY) has appeared so far at the LHC, so WIMPS particles DM are yet elusive ${ }^{15}$, while a di-photon excess decay at $750 \mathrm{GeV}$, rumoured at $3.9 \sigma$ significance is still debated. The claimed DAMA/LIBRA/CoGeNT annual modulation continues to be the only, although controversial, news from direct searches and similarly no incontrovertible indirect evidence of DM has been presented, to date.

Conflicts of Interest: The authors declare no conflict of interest.

\section{Bibliography}

1. Spergel, D.N.; Verde, L.; Peiris, H.V.; Komatsu, E.; Nolta, M.R.; Bennett, C.L.; Halpern, M.; Hinshaw, G.; Jarosik, N.; Kogut, A.; et al. First-Year Wilkinson Microwave Anisotropy Probe (WMAP) Observations: Determination of Cosmological Parameters. Astrophys. J. Supp. 2003, 148, 175-194.

2. Komatsu, E.; Smith, K.M.; Dunkley, J.; Bennett, C.L.; Gold, B.; Hinshaw, G.; Jarosik, N.; Larson, D.; Nolta, M.R.; Page, L.; et al. Seven-year Wilkinson Microwave Anisotropy Probe (WMAP) Observations: Cosmological Interpretation. Astrophys. J. Supp. 2011, 192, 18.

3. Del Popolo, A. Dark matter, density perturbations, and structure formation. Astron. Rep. 2007, 51, 169-196.

4. Del Popolo, A. Non-Baryonic Dark Matter in Cosmology; American Institute of Physics Conference Series; Urena-López, L.A., Becerril-Bárcenas, R., Linares-Romero, R., Eds.; American Institute of Physics: College Park, MD, USA, 2013; Volume 1548, pp. 2-63.

5. Del Popolo, A. Nonbaryonic Dark Matter in Cosmology. Int. J. Mod. Phys. D 2014, 23, 1430005.

6. Smith, K.M.; Zahn, O.; Doré, O. Detection of gravitational lensing in the cosmic microwave background. Phys. Rev. D 2007, 76, 043510.

7. Das, S.; Sherwin, B.D.; Aguirre, P.; Appel, J.W.; Bond, J.R.; Carvalho, C.S.; Devlin, M.J.; Dunkley, J.; Dünner, R.; Essinger-Hileman, T.; et al. Detection of the Power Spectrum of Cosmic Microwave Background Lensing by the Atacama Cosmology Telescope. Phys. Rev. Lett. 2011, 107, 021301.

8. Hanson, D.; Hoover, S.; Crites, A.; Ade, P.A.R.; Aird, K.A.; Austermann, J.E.; Beall, J.A.; Bender, A.N.; Benson, B.A.; Bleem, L.E.; et al. Detection of B-Mode Polarization in the Cosmic Microwave Background with Data from the South Pole Telescope. Phys. Rev. Lett. 2013, 111, 141301.

9. Weinberg, S. The cosmological constant problem. Rev. Mod. Phys. 1989, 61, 1-23.

10. Astashenok, A.V.; Del Popolo, A. Cosmological measure with volume averaging and the vacuum energy problem. Class. Quantum Gravity 2012, 29, 085014.

11. Martin, J. Everything You always Wanted to Know about the Cosmological Constant Problem (but Were Afraid to Ask). Comptes Rendus Phys. 2012, 13, 566-665.

12. Sivanandam, N. Is the Cosmological Coincidence a Problem? Phys. Rev. D 2013, 87, 083514.

13. Planck Collaboration; Ade, P.A.R.; Aghanim, N.; Armitage-Caplan, C.; Arnaud, M.; Ashdown, M.; Atrio-Barandela, F.; Aumont, J.; Baccigalupi, C.; Banday, A.J.; et al. Planck 2013 results. XVI. Cosmological parameters. Astron. Astrophys. 2014, 571, A16.

14. Raveri, M. Is There Concordance within the Concordance $\Lambda$ CDM Model? arXiv 2015, arXiv:1510.00688.

15. Macaulay, E.; Wehus, I.K.; Eriksen, H.K. Lower Growth Rate from Recent Redshift Space Distortion Measurements than Expected from Planck. Phys. Rev. Lett. 2013, 111, 161301.

16. Schwarz, D.J.; Starkman, G.D.; Huterer, D.; Copi, C.J. Is the Low-l Microwave Background Cosmic? Phys. Rev. Lett. 2004, 93, 221301.

17. Copi, C.J.; Huterer, D.; Schwarz, D.J.; Starkman, G.D. On the large-angle anomalies of the microwave sky. Mon. Not. Roy. Astro. Soc. 2006, 367, 79-102.

18. Copi, C.J.; Huterer, D.; Schwarz, D.J.; Starkman, G.D. Uncorrelated universe: Statistical anisotropy and the vanishing angular correlation function in WMAP years 13. Phys. Rev. D 2007, 75, 023507.

19. Copi, C.J.; Huterer, D.; Schwarz, D.J.; Starkman, G.D. Large-Angle Anomalies in the CMB. Adv. Astron. 2010, $2010,847541$.

15 The diphoton excess might be able to rescue the WIMP hypothesis. 
20. Copi, C.J.; Huterer, D.; Schwarz, D.J.; Starkman, G.D. Large-scale alignments from WMAP and Planck. Mon. Not. Roy. Astro. Soc. 2015, 449, 3458-3470.

21. Eriksen, H.K.; Hansen, F.K.; Banday, A.J.; Górski, K.M.; Lilje, P.B. Asymmetries in the Cosmic Microwave Background Anisotropy Field. Astrophys. J. 2004, 605, 14-20.

22. Hansen, F.K.; Banday, A.J.; Górski, K.M. Testing the cosmological principle of isotropy: Local power-spectrum estimates of the WMAP data. Mon. Not. Roy. Astro. Soc. 2004, 354, 641-665.

23. Jaffe, T.R.; Banday, A.J.; Eriksen, H.K.; Górski, K.M.; Hansen, F.K. Evidence of Vorticity and Shear at Large Angular Scales in the WMAP Data: A Violation of Cosmological Isotropy? Astrophys. J. Lett. 2005, 629, L1-L4.

24. Hoftuft, J.; Eriksen, H.K.; Banday, A.J.; Górski, K.M.; Hansen, F.K.; Lilje, P.B. Increasing Evidence for Hemispherical Power Asymmetry in the Five-Year WMAP Data. Astrophys. J. 2009, 699, 985-989.

25. Planck Collaboration; Ade, P.A.R.; Aghanim, N.; Armitage-Caplan, C.; Arnaud, M.; Ashdown, M.; Atrio-Barandela, F.; Aumont, J.; Baccigalupi, C.; Banday, A.J.; et al. Planck 2013 results. XXIII. Isotropy and statistics of the CMB. Astron. Astrophys. 2014, 571, A23.

26. Akrami, Y.; Fantaye, Y.; Shafieloo, A.; Eriksen, H.K.; Hansen, F.K.; Banday, A.J.; Górski, K.M. Power Asymmetry in WMAP and Planck Temperature Sky Maps as Measured by a Local Variance Estimator. Astrophys. J. Lett. 2014, 784, L42.

27. Cruz, M.; Martínez-González, E.; Vielva, P.; Cayón, L. Detection of a non-Gaussian spot in WMAP. Mon. Not. Roy. Astro. Soc. 2005, 356, 29-40.

28. Cruz, M.; Tucci, M.; Martínez-González, E.; Vielva, P. The non-Gaussian cold spot in Wilkinson Microwave Anisotropy Probe: Significance, morphology and foreground contribution. Mon. Not. Roy. Astro. Soc. 2006, $369,57-67$.

29. Cruz, M.; Cayón, L.; Martínez-González, E.; Vielva, P.; Jin, J. The Non-Gaussian Cold Spot in the 3 Year Wilkinson Microwave Anisotropy Probe Data. Astrophys. J. 2007, 655, 11-20.

30. Bennett, C.L.; Hill, R.S.; Hinshaw, G.; Larson, D.; Smith, K.M.; Dunkley, J.; Gold, B.; Halpern, M.; Jarosik, N.; Kogut, A.; et al. Seven-year Wilkinson Microwave Anisotropy Probe (WMAP) Observations: Are There Cosmic Microwave Background Anomalies? Astrophys. J. Supp. 2011, 192, 17.

31. Moore, B. Evidence against dissipation-less dark matter from observations of galaxy haloes. Nature 1994, 370, 629-631.

32. Moore, B.; Quinn, T.; Governato, F.; Stadel, J.; Lake, G. Cold collapse and the core catastrophe. Mon. Not. Roy. Astro. Soc. 1999, 310, 1147-1152.

33. Ostriker, J.P.; Steinhardt, P. New Light on Dark Matter. Science 2003, 300, 1909-1914.

34. Boylan-Kolchin, M.; Bullock, J.S.; Kaplinghat, M. Too big to fail? The puzzling darkness of massive Milky Way subhaloes. Mon. Not. Roy. Astro. Soc. 2011, 415, L40-L44.

35. Boylan-Kolchin, M.; Bullock, J.S.; Kaplinghat, M. The Milky Way's bright satellites as an apparent failure of $\Lambda$ CDM. Mon. Not. Roy. Astro. Soc. 2012, 422, 1203-1218.

36. Oh, S.H.; Brook, C.; Governato, F.; Brinks, E.; Mayer, L.; de Blok, W.J.G.; Brooks, A.; Walter, F. The Central Slope of Dark Matter Cores in Dwarf Galaxies: Simulations versus THINGS. Astro. J. 2011, 142, 24.

37. Flores, R.A.; Primack, J.R. Observational and theoretical constraints on singular dark matter halos. Astrophys. J. Lett. 1994, 427, L1-L4.

38. Navarro, J.F.; Frenk, C.S.; White, S.D.M. The Structure of Cold Dark Matter Halos. Astrophys. J. 1996, $462,563$.

39. Navarro, J.F.; Ludlow, A.; Springel, V.; Wang, J.; Vogelsberger, M.; White, S.D.M.; Jenkins, A.; Frenk, C.S.; Helmi, A. The diversity and similarity of simulated cold dark matter haloes. Mon. Not. Roy. Astro. Soc. 2010, 402, 21-34.

40. Saburova, A.; Del Popolo, A. On the surface density of dark matter haloes. Mon. Not. R. Astron. Soc. 2014, $445,3512-3524$

41. Klypin, A.A.; Kravtsov, A.V.; Valenzuela, O.; Prada, F. Where are the missing Galactic satellites? Astrophys. J. 1999, 522, 82-92.

42. Van den Bosch, F.C.; Burkert, A.; Swaters, R.A. The angular momentum content of dwarf galaxies: New challenges for the theory of galaxy formation. Mon. Not. Roy. Astro. Soc. 2001, 326, 1205-1215.

43. Cardone, V.F.; Del Popolo, A.; Kroupa, P. Angular momentum transfer and the size - mass relation in early type galaxies. Mon. Not. R. Astron. Soc. 2009, 400, 766-774. 
44. Pawlowski, M.S.; Famaey, B.; Jerjen, H.; Merritt, D.; Kroupa, P.; Dabringhausen, J.; Lüghausen, F.; Forbes, D.A.; Hensler, G.; Hammer, F.; et al. Co-orbiting satellite galaxy structures are still in conflict with the distribution of primordial dwarf galaxies. Mon. Not. Roy. Astro. Soc. 2014, 442, 2362-2380.

45. McGaugh, S.S. Novel Test of Modified Newtonian Dynamics with Gas Rich Galaxies. Phys. Rev. Lett. 2011, 106, 121303.

46. Kroupa, P.; Theis, C.; Boily, C.M. The Great disk of Milky Way satellites and cosmological sub-structures. Astron. Astrophys. 2005, 431, 517-521.

47. Kroupa, P.; Famaey, B.; de Boer, K.S.; Dabringhausen, J.; Pawlowski, M.S.; Boily, C.M.; Jerjen, H.; Forbes, D.; Hensler, G.; Metz, M. Local-Group tests of dark-matter Concordance Cosmology: Towards a new paradigm for structure formation? Astron. Astrophys. 2010, 523, A32.

48. Kroupa, P. The dark matter crisis: Falsification of the current standard model of cosmology. Publ. Astron. Soc. Aust. 2012, 29, 395-433.

49. Kroupa, P.; Pawlowski, M.; Milgrom, M. The failures of the standard model of cosmology require a new paradigm. Int. J. Mod. Phys. D 2012, 21, 1230003.

50. Kroupa, P. Galaxies as simple dynamical systems: Observational data disfavor dark matter and stochastic star formation. Can. J. Phys. 2015, 93, 169-202.

51. Navarro, J.F.; Benz, W. Dynamics of cooling gas in galactic dark halos. Astrophys. J. 1991, 380, 320-329.

52. Navarro, J.F.; Steinmetz, M. The Effects of a Photoionizing Ultraviolet Background on the Formation of Disk Galaxies. Astrophys. J. 1997, 478, 13-28.

53. Sommer-Larsen, J.; Gelato, S.; Vedel, H. Formation of Disk Galaxies: Feedback and the Angular Momentum Problem. Astrophys. J. 1999, 519, 501-512.

54. Navarro, J.F.; Steinmetz, M. Dark Halo and Disk Galaxy Scaling Laws in Hierarchical Universes. Astrophys. J. 2000, 538, 477-488.

55. Maller, A.H.; Dekel, A. Towards a resolution of the galactic spin crisis: Mergers, feedback and spin segregation. Mon. Not. Roy. Astro. Soc. 2002, 335, 487-498.

56. Sommer-Larsen, J.; Götz, M.; Portinari, L. Galaxy Formation: Cold Dark Matter, Feedback, and the Hubble Sequence. Astrophys. J. 2003, 596, 47-66.

57. Abadi, M.G.; Navarro, J.F.; Steinmetz, M.; Eke, V.R. Simulations of Galaxy Formation in a $\Lambda$ Cold Dark Matter Universe. I. Dynamical and Photometric Properties of a Simulated Disk Galaxy. Astrophys. J. 2003, 591, 499-514.

58. Governato, F.; Brook, C.; Mayer, L.; Brooks, A.; Rhee, G.; Wadsley, J.; Jonsson, P.; Willman, B.; Stinson, G.; Quinn, T.; et al. Bulgeless dwarf galaxies and dark matter cores from supernova-driven outflows. Nature 2010, 463, 203-206.

59. Del Popolo, A.; Lima, J.A.S.; Fabris, J.C.; Rodrigues, D.C. A unified solution to the small scale problems of the $\Lambda$ CDM model. J. Cosmo. Astrop. Phys 2014, 4, 021.

60. Tully, R.B.; Fisher, J.R. A new method of determining distances to galaxies. Astron. Astrophys. 1977, 54, 661-673.

61. Bell, E.F.; de Jong, R.S. Stellar mass-to-light ratios and the Tully-Fisher relation. Astrophys. J. 2001, 550, 212-229.

62. Verheijen, M.A.W. The Ursa Major Cluster of Galaxies. 5. H I Rotation Curve Shapes and the Tully-Fisher Relations. Astrophys. J. 2001, 563, 694-715.

63. Gurovich, S.; McGaugh, S.S.; Freeman, K.C.; Jerjen, H.; Staveley-Smith, L.; De Blok, W.J.G. The Baryonic Tully Fisher relation. Publ. Astron. Soc. Aust. 2004, 21, 412.

64. McGaugh, S.S. The Baryonic Tully-Fisher relation of galaxies with extended rotation curves and the stellar mass of rotating galaxies. Astrophys. J. 2005, 632, 859-871.

65. Pfenniger, D.; Revaz, Y. The Baryonic Tully-Fisher relation revisited. Astron. Astrophys. 2005, 431, 511.

66. Begum, A.; Chengalur, J.N.; Karachentsev, I.D.; Sharina, M.E. Baryonic Tully-Fisher Relation for Extremely Low Mass Galaxies. Mon. Not. R. Astron. Soc. 2008, 386, 138.

67. Stark, D.V.; McGaugh, S.S.; Swaters, R.A. A First Attempt to Calibrate the Baryonic Tully-Fisher Relation with Gas Dominated Galaxies. Astron. J. 2009, 138, 392.

68. Trachternach, C.; de Blok, W.J.G.; McGaugh, S.S.; van der Hulst, J.M.; Dettmar, R.J. The baryonic Tully-Fisher relation and its implication for dark matter halos. Astron. Astrophys. 2009, 505, 577. 
69. Gurovich, S.; Freeman, K.; Jerjen, H.; Staveley-Smith, L.; Puerari, I. The slope of the Baryonic Tully-Fisher relation. Astron. J. 2010, 140, 663.

70. McGaugh, S. The Baryonic Tully-Fisher Relation of Gas Rich Galaxies as a Test of LCDM and MOND. Astron. J. 2012, 143, 40.

71. Sawala, T.; Frenk, C.S.; Fattahi, A.; Navarro, J.F.; Theuns, T.; Bower, R.G.; Crain, R.A.; Furlong, M.; Jenkins, A.; Schaller, M.; et al. The chosen few: The low-mass haloes that host faint galaxies. Mon. Not. Roy. Astro. Soc. 2016, 456, 85-97.

72. Pawlowski, M.S.; Famaey, B.; Merritt, D.; Kroupa, P. On the Persistence of Two Small-scale Problems in

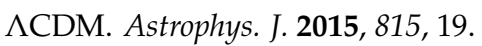

73. Zolotov, A.; Brooks, A.M.; Willman, B.; Governato, F.; Pontzen, A.; Christensen, C.; Dekel, A.; Quinn, T.; Shen, S.; Wadsley, J. Baryons Matter: Why Luminous Satellite Galaxies have Reduced Central Masses. Astrophys. J. 2012, 761, 71.

74. Brooks, A.M.; Zolotov, A. Why Baryons Matter: The Kinematics of Dwarf Spheroidal Satellites. Astrophys. J. 2014, 786, 87.

75. Mashchenko, S.; Couchman, H.M.P.; Wadsley, J. The removal of cusps from galaxy centres by stellar feedback in the early Universe. Nature 2006, 442, 539-542.

76. Mashchenko, S.; Wadsley, J.; Couchman, H.M.P. Stellar Feedback in Dwarf Galaxy Formation. Science 2008, 319, 174.

77. Peñarrubia, J.; Benson, A.J.; Walker, M.G.; Gilmore, G.; McConnachie, A.W.; Mayer, L. The impact of dark matter cusps and cores on the satellite galaxy population around spiral galaxies. Mon. Not. Roy. Astro. Soc. 2010, 406, 1290-1305.

78. Kunkel, W.E.; Demers, S. The Magellanic Plane. In The Galaxy and the Local Group; Royal Greenwich Observatory Bulletins: Royal Observatory Greenwich, London, UK, 1976; Volume 182, p. 241.

79. Lynden-Bell, D. Dwarf galaxies and globular clusters in high velocity hydrogen streams. Mon. Not. R. Astron. Soc. 1976, 174, 695.

80. Metz, M.; Kroupa, P.; Libeskind, N.I. The orbital poles of Milky Way satellite galaxies: A rotationally supported disc-of-satellites. Astrophys. J. 2008, 680, 287-294.

81. Pawlowski, M.S.; Kroupa, P. The rotationally stabilized VPOS and predicted proper motions of the Milky Way satellite galaxies. Mon. Not. R. Astron. Soc. 2013, 435, 2116.

82. Ibata, R.A.; Lewis, G.F.; Conn, A.R.; Irwin, M.J.; McConnachie, A.W.; Chapman, S.C.; Collins, M.L.; Fardal, M.; Ferguson, A.M.N.; Ibata, N.G.; et al. A Vast Thin Plane of Co-rotating Dwarf Galaxies Orbiting the Andromeda Galaxy. Nature 2013, 493, 62-65.

83. Metz, M.; Kroupa, P.; Jerjen, H. The spatial distribution of the Milky Way and Andromeda satellite galaxies. Mon. Not. R. Astron. Soc. 2007, 374, 1125-1145.

84. McConnachie, A.; Irwin, M. The satellite distribution of m31. Mon. Not. R. Astron. Soc. 2006, 365, 902-914.

85. Koch, A.; Grebel, E.K. The anisotropic distribution of $\mathrm{m} 31$ satellite galaxies: A polar great plane of early-type companions. Astron. J. 2006, 131, 1405-1415.

86. Conn, A.R.; Lewis, G.F.; Ibata, R.A.; Parker, Q.A.; Zucker, D.B.; McConnachie, A.W.; Martin, N.F.; Valls-Gabaud, D.; Tanvir, N.; Irwin, M.J.; et al. The Three-Dimensional Structure of the M31 Satellite System: Strong Evidence for an Inhomogeneous Distribution of Satellites. Astrophys. J. 2013, 766, 120.

87. Shaya, E.J.; Tully, R.B. The Formation of the Local Group Planes of Galaxies. Mon. Not. R. Astron. Soc. 2013, 436, 2096-2119.

88. Tully, R.B.; Libeskind, N.I.; Karachentsev, I.D.; Karachentseva, V.E.; Rizzi, L.; Shaya, E.J. Two Planes of Satellites in the Centaurus A Group. Astrophys. J. Lett. 2015, 802, L25.

89. Bowden, A.; Evans, N.W.; Belokurov, V. On Asymmetric Distributions of Satellite Galaxies. Astrophys. J. 2014, 793, L42.

90. Cautun, M.; Wang, W.; Frenk, C.S.; Sawala, T. A new spin on discs of satellite galaxies. Mon. Not. R. Astron. Soc. 2015, 449, 2576-2587.

91. Libeskind, N.I.; Frenk, C.S.; Cole, S.; Helly, J.C.; Jenkins, A.; Navarro, J.F.; Power, C. The Distribution of satellite galaxies: The Great pancake. Mon. Not. R. Astron. Soc. 2005, 363, 146-152.

92. Gillet, N.; Ocvirk, P.; Aubert, D.; Knebe, A.; Libeskind, N.; Yepes, G.; Gottlöber, S.; Hoffman, Y. Vast Planes of Satellites in a High-resolution Simulation of the Local Group: Comparison to Andromeda. Astrophys. J. 2015, 800, 34 . 
93. Hammer, F.; Yang, Y.; Fouquet, S.; Pawlowski, M.S.; Kroupa, P.; Puech, M.; Flores, H.; Wang, J. The vast thin plane of M31 co-rotating dwarfs: An additional fossil signature of the M31 merger and of its considerable impact in the whole Local Group. Mon. Not. R. Astron. Soc. 2013, 431, 3543.

94. Smith, R.; Duc, P.A.; Candlish, G.N.; Fellhauer, M.; Sheen, Y.K.; Gibson, B.K. The Influence of Ram Pressure on the Evolution of Tidal Dwarf Galaxies. Mon. Not. R. Astron. Soc. 2013, 436, 839.

95. Pawlowski, M.S.; Kroupa, P.; Jerjen, H. Dwarf Galaxy Planes: The discovery of symmetric structures in the Local Group. Mon. Not. R. Astron. Soc. 2013, 435, 1928.

96. Bellazzini, M.; Oosterloo, T.; Fraternali, F.; Beccari, G. Dwarfs walking in a row. The filamentary nature of the NGC3109 association. Astron. Astrophys. 2013, 559, L11.

97. Pawlowski, M.S.; McGaugh, S.S. Perseus I and the NGC 3109 association in the context of the Local Group dwarf galaxy structures. Mon. Not. R. Astron. Soc. 2014, 440, 908-919.

98. Galianni, P.; Patat, F.; Higdon, J. L.; Mieske, S.; Kroupa, P. VLT observations of NGC 1097's “dog-leg" tidal stream. Dwarf spheroidals and tidal streams. Astron. Astrophys. 2010, 521, A20.

99. Duc, P.A.; Paudel, S.; McDermid, R.M.; Cuillandre, J.C.; Serra, P.; Bournaud, F.; Cappellari, M.; Emsellem, E. Identification of old tidal dwarfs near early-type galaxies from deep imaging and H I observations. Mon. Not. R. Astron. Soc. 2014, 440, 1458-1469.

100. Paudel, S.; Duc, P.-A.; Cote, P.; Cuillandre, J.-C.; Ferrarese, L.; Ferriere, E.; Gwyn, S.D.J.; Mihos, J.C.; Vollmer, B.; Balogh, M.L.; et al. The Next Generation Virgo Cluster Survey. IV. NGC 4216: A Bombarded Spiral in the Virgo Cluster. Astrophys. J. 2013, 767, 133.

101. Karachentsev, I.D.; Bautzmann, D.; Neyer, F.; Polzl, R.; Riepe, P.; Zilch, T.; Mattern, B. Three Low Surface Brightness Dwarfs Discovered around NGC 4631. arXiv 2014, arXiv:1401.2719.

102. Barnes, J.E.; Lars, H. Formation of dwarf galaxies in tidal tails. Nature 1992, 360, 715-717.

103. Bournaud, F.; Duc, P.A.; Emsellem, E. High-resolution simulations of galaxy mergers: resolving globular cluster formation. Mon. Not. R. Astron. Soc. 2008, 389, L8-L12.

104. Sawa, T.; Fujimoto, M. A Dynamical Model for the Orbit of the Andromeda Galaxy M31 and the Origin of the Local Group of Galaxies. Pub. Astron. Soc. Jpn 2005, 57, 429-446.

105. Pawlowski, M.S.; Pflamm-Altenburg, J.; Kroupa, P. The VPOS: A vast polar structure of satellite galaxies, globular clusters and streams around the Milky Way. Mon. Not. R. Astron. Soc. 2012, 423, 1109.

106. Zhao, H.; Famaey, B.; Lüghausen, F.; Kroupa, P. Local Group timing in Milgromian dynamics. A past Milky Way-Andromeda encounter at z $>0.8$. Astron. Astrophys. 2013, 557, L3.

107. Pawlowski, M.S.; Kroupa, P.; de Boer, K.S. Making Counter-Orbiting Tidal Debris: The Origin of the Milky Way Disc of Satellites. Astron. Astrophys. 2011, 532, A118.

108. Yang, Y.; Hammer, F. Could the Magellanic Clouds be tidal dwarves expelled from a past-merger event occurring in Andromeda? Astrophys. J. 2010, 725, L24.

109. Fouquet, S.; Hammer, F.; Yang, Y.; Puech, M.; Flores, H. Does the dwarf galaxy system of the Milky Way originate from Andromeda? Mon. Not. R. Astron. Soc. 2012, 427, 1769.

110. Lake, G.; D'Onghia, E. Small Dwarf Galaxies Within Larger Dwarfs: Why Some Are Luminous While Most Go Dark. Astrophys. J. 2008, 686, L61.

111. Li, Y.S.; Helmi, A. Infall of Substructures onto a Milky Way-like Dark Halo. Mon. Not. R. Astron. Soc. 2008, 385, 1365.

112. Metz, M.; Kroupa, P.; Theis, C.; Hensler, G.; Jerjen, H. Did the Milky Way dwarf satellites enter the halo as a group? Astrophys. J. 2009, 697, 269-274.

113. Lovell, M.; Eke, V.; Frenk, C.; Jenkins, A. The Link between Galactic Satellite Orbits and Subhalo Accretion. Mon. Not. R. Astron. Soc. 2011, 413, 3013.

114. Libeskind, N.I.; Frenk, C.S.; Cole, S.; Jenkins, A.; Helly, J.C. How common is the Milky Way-Satellite system alignment? Mon. Not. R. Astron. Soc. 2009, 399, 550.

115. Deason, A.J.; McCarthy, I.G.; Font, A.; Evans, N.W.; Frenk, C.S.; Belokurov, V.; Libeskind, N.I.; Crain, R.A.; Theuns, T. Mismatch and Misalignment: Dark Haloes and Satellites of Disc Galaxies. Mon. Not. R. Astron. Soc. 2011, 415, 2607.

116. Bahl, H.; Baumgardt, H. A comparison of the distribution of satellite galaxies around Andromeda and the results of $\Lambda$ CDM simulations. Mon. Not. R. Astron. Soc. 2014, 438, 2916-2923.

117. Wang, J.; Frenk, C.S.; Cooper, A.P. The Spatial Distribution of Galactic Satellites in the LCDM Cosmology. Mon. Not. R. Astron. Soc. 2013, 429, 1502. 
118. Goerdt, T.; Burkert, A. The Co-Planarity of Satellite Galaxies Delivered by Randomly Aligned Cold Mode Accretion Streams. arXiv 2013, arXiv:1307.2102.

119. Sawala, T.; Fren, C.S.; Fattahi, A.; Navarro, J.F.; Bower, R.G.; Crain, R.A.; Vecchia, C.D.; Furlong, M.; Helly, J.C.; Jenkins, A.; et al. The APOSTLE simulations: Solutions to the Local Group's cosmic puzzles. Mon. Not. R. Astron. Soc. 2016, 457, 1931-1943.

120. McGaugh, S.S.; Schombert, J.M.; Bothun, G.D.; de Blok, W.J.G. The Baryonic Tully-Fisher relation. Astrophys. J. 2000, 533, L99-L102.

121. Torres-Flores, S.; Epinat, B.; Amram, P.; Plana, H.; de Oliveira, C.M. GHASP: An $\mathrm{H}_{\alpha}$ kinematic survey of spiral and irregular galaxies-IX. The NIR, stellar and baryonic Tully-Fisher relations. Mon. Not. R. Astron. Soc. 2011, 416, 1936.

122. Lelli, F.; McGaugh, S.S.; Schombert, J.M. The Small Scatter of the Baryonic Tully-fisher Relation. Astrophys. J. 2016, 816, L14.

123. Mo, H.J.; Mao, S.; White, S.D.M. The Formation of galactic disks. Mon. Not. R. Astron. Soc. 1998, $295,319$.

124. Steinmetz, M.; Navarro, J. The cosmological origin of the tully-fisher relation. Astrophys. J. 1999, 513, 555-560.

125. White, S.D.M.; Frenk, C.S. Galaxy formation through hierarchical clustering. Astrophys. J. 1991, 379, 52-79.

126. Dutton, A.A. The baryonic Tully-Fisher relation and galactic outflows. Mon. Not. R. Astron. Soc. 2012, 424, 3123.

127. Desmond, H.; Wechsler, R.H. The Tully-Fisher and mass-size relations from halo abundance matching. Mon. Not. R. Astron. Soc. 2015, 454, 322-343.

128. Navarro, J.F.; Steinmetz, M. The core density of dark matter halos: A critical challenge to the lambda-cdm paradigm? Astrophys. J. 2000, 528, 607-611.

129. Scannapieco, C.; Wadepuhl, M.; Parry, O.H.; Navarro, J.F.; Jenkins, A.; Springel, V.; Teyssier, R.; Carlson, E.; Couchman, H.M.P.; Crain, R.A.; et al. The Aquila comparison Project: The Effects of Feedback and Numerical Methods on Simulations of Galaxy Formation. Mon. Not. R. Astron. Soc. 2012, 423, 1726.

130. Cole, S.; Lacey, C.G.; Baugh, C.M.; Frenk, C.S. Hierarchical galaxy formation. Mon. Not. R. Astron. Soc. 2000, $319,168$.

131. Guedes, J.; Callegari, S.; Madau, P.; Mayer, L. Forming Realistic Late-Type Spirals in a LCDM Universe: The Eris Simulation. Astrophys. J. 2011, 742, 76.

132. Brook, C.B.; Stinson, G.; Gibson, B.K.; Wadsley, J.; Quinn, T. MaGICC Disks: Matching Observed Galaxy Relationships Over a Wide Stellar Mass Range. Mon. Not. R. Astron. Soc. 2012, 424, 1275.

133. McCarthy, I.G.; Schaye, J.; Font, A.S.; Theuns, T.; Frenk, C.S.; Crain, R.A.; Vecchia, C.D. Rotation rates, sizes, and star formation efficiencies of a representative population of simulated disc galaxies. Mon. Not. R. Astron. Soc. 2012, 427, 379-392.

134. Aumer, M.; White, S.; Naab, T.; Scannapieco, C. Towards a more realistic population of bright spiral galaxies in cosmological simulations. Mon. Not. R. Astron. Soc. 2013, 434, 3142-3164.

135. Marinacci, F.; Pakmor, R.; Springel, V. The formation of disc galaxies in high-resolution moving-mesh cosmological simulations. Mon. Not. Roy. Astro. Soc. 2014, 437, 1750-1775.

136. Dutton, A.A.; Bosch, F.C.V.D. The Impact of Feedback on Disk Galaxy Scaling Relations. Mon. Not. R. Astron. Soc. 2009, 396, 141.

137. Chan, T.K.; Kereš, D.; Oñorbe, J.; Hopkins, P.F.; Muratov, A.L.; Faucher-Giguère, C.A.; Quataert, E. The impact of baryonic physics on the structure of dark matter haloes: The view from the FIRE cosmological simulations. Mon. Not. Roy. Astro. Soc. 2015, 454, 2981-3001.

138. Vogelsberger, M.; Zavala, J.; Simpson, C.; Jenkins, A. Dwarf galaxies in CDM and SIDM with baryons: Observational probes of the nature of dark matter. Mon. Not. R. Astron. Soc. 2014, 444, 3684.

139. Schaller, M.; Frenk, C.S.; Bower, R.G.; Theuns, T.; Jenkins, A.; Schaye, J.; Crain, R.A.; Furlong, M.; Dalla Vecchia, C.; McCarthy, I.G. Baryon effects on the internal structure of $\Lambda$ CDM haloes in the EAGLE simulations. Mon. Not. Roy. Astro. Soc. 2015, 451, 1247-1267.

140. Schaye, J.; Crain, R.A.; Bower, R.G.; Furlong, M.; Schaller, M.; Theuns, T.; Vecchia, C.D.; Frenk, C.S.; McCarthy, I.G.; Helly, J.C.; et al. The EAGLE project: Simulating the evolution and assembly of galaxies and their environments. Mon. Not. R. Astron. Soc. 2015, 446, 521-554.

141. Crain, R.A.; Schaye, J.; Bower, R.G.; Furlong, M.; Schaller, M.; Theuns, T.; Vecchia, C.D.; Frenk, C.S.; McCarthy, I.G.; Helly, J.C.; et al. The EAGLE simulations of galaxy formation: Calibration of subgrid physics and model variations. Mon. Not. R. Astron. Soc. 2015, 450, 1937-1961. 
142. Fattahi, A.; Navarro, J.F.; Sawala, T.; Frenk, C.S.; Oman, K.A.; Crain, R.A.; Furlong, M.; Schaller, M.; Schaye, J.; Theuns, T.; et al. The APOSTLE project: Local Group kinematic mass constraints and simulation candidate selection. Mon. Not. Roy. Astro. Soc. 2016, 457, 844-856.

143. Sales, L.V.; Navarro, J.F.; Oman, K.; Fattahi, A.; Ferrero, I.; Abadi, M.G.; Bower, R.; Crain, R.A.; Frenk, C.S.; Sawala, T.; et al. The Low-Mass End of the Baryonic Tully-Fisher Relation. arXiv 2016, arXiv:1602.02155.

144. Geha, M.; Blanton, M.R.; Masjedi, M.; West, A.A. The Baryon Content of Extremely Low Mass Dwarf Galaxies. Astrophys. J. 2006, 653, 240-254.

145. Di Cintio, A.; Lelli, F. The mass discrepancy acceleration relation in a $\Lambda$ CDM context. Mon. Not. R. Astron. Soc. 2016, 456, L127-L131.

146. Navarro, J.F.; Frenk, C.S.; White, S.D.M. A Universal Density Profile from Hierarchical Clustering. Astrophys. J. 1997, 490, 493-508.

147. Moore, B.; Governato, F.; Quinn, T.; Stadel, J.; Lake, G. Resolving the Structure of Cold Dark Matter Halos. Astrophys. J. Lett. 1998, 499, L5-L8.

148. Fukushige, T.; Makino, J. Structure of Dark Matter Halos from Hierarchical Clustering. Astrophys. J. 2001, 557, 533-545.

149. Jing, Y.P.; Suto, Y. The Density Profiles of the Dark Matter Halo Are Not Universal. Astrophys. J. Lett. 2000, 529, L69-L72.

150. Ricotti, M. Dependence of the inner dark matter profile on the halo mass. Mon. Not. Roy. Astro. Soc. 2003, $344,1237-1249$.

151. Ricotti, M.; Wilkinson, M.I. On the origin of dark matter cores in dwarf galaxies. Mon. Not. Roy. Astro. Soc. 2004, 353, 867-873.

152. Ricotti, M.; Pontzen, A.; Viel, M. Is the Concentration of Dark Matter Halos at Virialization Universal? Astrophys. J. Lett. 2007, 663, L53-L56.

153. Del Popolo, A. On the universality of density profiles. Mon. Not. Roy. Astro. Soc. 2010, 408, 1808-1817.

154. Cardone, V.F.; Del Popolo, A.; Tortora, C.; Napolitano, N.R. Secondary infall model and dark matter scaling relations in intermediate-redshift early-type galaxies. Mon. Not. Roy. Astro. Soc. 2011, 416, 1822-1835.

155. Del Popolo, A. Non-power law behavior of the radial profile of phase-space density of halos. J. Cosmo. Astrop. Phys 2011, 7, 014.

156. Del Popolo, A.; Cardone, V.F.; Belvedere, G. Surface density of dark matter haloes on galactic and cluster scales. Mon. Not. Roy. Astro. Soc. 2013, 429, 1080-1087.

157. Di Cintio, A.; Brook, C.B.; Macciò, A.V.; Stinson, G.S.; Knebe, A.; Dutton, A.A.; Wadsley, J. The dependence of dark matter profiles on the stellar-to-halo mass ratio: A prediction for cusps versus cores. Mon. Not. Roy. Astro. Soc. 2014, 437, 415-423.

158. Stadel, J.; Potter, D.; Moore, B.; Diemand, J.; Madau, P.; Zemp, M.; Kuhlen, M.; Quilis, V. Quantifying the heart of darkness with GHALO - a multibillion particle simulation of a galactic halo. Mon. Not. Roy. Astro. Soc. 2009, 398, L21-L25.

159. Gao, L.; Navarro, J.F.; Cole, S.; Frenk, C.S.; White, S.D.M.; Springel, V.; Jenkins, A.; Neto, A.F. The redshift dependence of the structure of massive $\Lambda$ cold dark matter haloes. Mon. Not. Roy. Astro. Soc. 2008, $387,536-544$.

160. Burkert, A. The Structure of Dark Matter Halos in Dwarf Galaxies. Astrophys. J. Lett. 1995, 447, L25.

161. De Blok, W.J.G.; Bosma, A.; McGaugh, S. Simulating observations of dark matter dominated galaxies: Towards the optimal halo profile. Mon. Not. Roy. Astro. Soc. 2003, 340, 657-678.

162. Swaters, R.A.; Madore, B.F.; van den Bosch, F.C.; Balcells, M. The Central Mass Distribution in Dwarf and Low Surface Brightness Galaxies. Astrophys. J. 2003, 583, 732-751.

163. Kuzio de Naray, R.; Kaufmann, T. Recovering cores and cusps in dark matter haloes using mock velocity field observations. Mon. Not. Roy. Astro. Soc. 2011, 414, 3617-3626.

164. Oh, S.H.; de Blok, W.J.G.; Brinks, E.; Walter, F.; Kennicutt, R.C., Jr. Dark and Luminous Matter in THINGS Dwarf Galaxies. Astro. J. 2011, 141, 193.

165. Governato, F.; Zolotov, A.; Pontzen, A.; Christensen, C.; Oh, S.H.; Brooks, A.M.; Quinn, T.; Shen, S.; Wadsley, J. Cuspy no more: How outflows affect the central dark matter and baryon distribution in $\Lambda$ cold dark matter galaxies. Mon. Not. Roy. Astro. Soc. 2012, 422, 1231-1240.

166. Del Popolo, A. The Cusp/Core Problem and the Secondary Infall Model. Astrophys. J. 2009, 698, $2093-2113$. 
167. Cardone, V.F.; Del Popolo, A. Newtonian acceleration scales in spiral galaxies. Mon. Not. Roy. Astro. Soc. 2012, 427, 3176-3187.

168. Del Popolo, A. Density profile slopes of dwarf galaxies and their environment. Mon. Not. Roy. Astro. Soc. 2012, 419, 971-984.

169. Del Popolo, A. On the density-profile slope of clusters of galaxies. Mon. Not. Roy. Astro. Soc. 2012, 424, 38-51.

170. Del Popolo, A.; Cardone, V.F. Statistical properties of the dark matter haloes of dwarf galaxies and correlations with the environment. Mon. Not. Roy. Astro. Soc. 2012, 423, 1060-1072.

171. Del Popolo, A.; Hiotelis, N. Cusps and cores in the presence of galactic bulges. J. Cosmo. Astrop. Phys 2014, 2014, 047.

172. Del Popolo, A. Profiles of dark-matter haloes at high redshift. Mon. Not. R. Astron. Soc. 2001, 325, 1190.

173. McGaugh, S.S.; de Blok, W.J.G. Testing the Dark Matter Hypothesis with Low Surface Brightness Galaxies and Other Evidence. Astrophys. J. 1998, 499, 41-65.

174. Côté, S.; Carignan, C.; Freeman, K.C. The Various Kinematics of Dwarf Irregular Galaxies in Nearby Groups and Their Dark Matter Distributions. Astro. J. 2000, 120, 3027-3059.

175. van den Bosch, F.C.; Swaters, R.A. Dwarf galaxy rotation curves and the core problem of dark matter haloes. Mon. Not. Roy. Astro. Soc. 2001, 325, 1017-1038.

176. De Blok, W.J.G.; Bosma, A. High-resolution rotation curves of low surface brightness galaxies. Astron. Astrophys. 2002, 385, 816-846.

177. Spekkens, K.; Giovanelli, R.; Haynes, M.P. The Cusp/Core Problem in Galactic Halos: Long-Slit Spectra for a Large Dwarf Galaxy Sample. Astro. J. 2005, 129, 2119-2137.

178. Hayashi, E.; Navarro, J.F.; Power, C.; Jenkins, A.; Frenk, C.S.; White, S.D.M.; Springel, V.; Stadel, J.; Quinn, T.R. The inner structure of $\Lambda \mathrm{CDM}$ haloes-II. Halo mass profiles and low surface brightness galaxy rotation curves. Mon. Not. Roy. Astro. Soc. 2004, 355, 794-812.

179. Blais-Ouellette, S.; Amram, P.; Carignan, C. Accurate Determination of the Mass Distribution in Spiral Galaxies. II. Testing the Shape of Dark Halos. Astro. J. 2001, 121, 1952-1964.

180. Kuzio de Naray, R.; McGaugh, S.S.; de Blok, W.J.G. Mass Models for Low Surface Brightness Galaxies with High-Resolution Optical Velocity Fields. Astrophys. J. 2008, 676, 920-943.

181. Kuzio de Naray, R.; McGaugh, S.S.; Mihos, J.C. Constraining the NFW Potential with Observations and Modeling of Low Surface Brightness Galaxy Velocity Fields. Astrophys. J. 2009, 692, 1321-1332.

182. Weldrake, D.T.F.; de Blok, W.J.G.; Walter, F. A high-resolution rotation curve of NGC 6822: A test-case for cold dark matter. Mon. Not. Roy. Astro. Soc. 2003, 340, 12-28.

183. Trachternach, C.; de Blok, W.J.G.; Walter, F.; Brinks, E.; Kennicutt, R.C., Jr. Dynamical Centers and Noncircular Motions in THINGS Galaxies: Implications for Dark Matter Halos. Astro. J. 2008, 136, 2720-2760.

184. Gentile, G.; Salucci, P.; Klein, U.; Vergani, D.; Kalberla, P. The cored distribution of dark matter in spiral galaxies. Mon. Not. Roy. Astro. Soc. 2004, 351, 903-922.

185. Gentile, G.; Salucci, P.; Klein, U.; Granato, G.L. NGC 3741: The dark halo profile from the most extended rotation curve. Mon. Not. Roy. Astro. Soc. 2007, 375, 199-212.

186. Salucci, P.; Lapi, A.; Tonini, C.; Gentile, G.; Yegorova, I.; Klein, U. The universal rotation curve of spiral galaxies - II. The dark matter distribution out to the virial radius. Mon. Not. Roy. Astro. Soc. 2007, 378, 41-47.

187. Navarro, J.F.; Hayashi, E.; Power, C.; Jenkins, A.R.; Frenk, C.S.; White, S.D.M.; Springel, V.; Stadel, J.; Quinn, T.R. The inner structure of $\Lambda$ CDM haloes-III. Universality and asymptotic slopes. Mon. Not. Roy. Astro. Soc. 2004, 349, 1039-1051.

188. Oh, S.H.; Hunter, D.A.; Brinks, E.; Elmegreen, B.G.; Schruba, A.; Walter, F.; Rupen, M.P.; Young, L.M.; Simpson, C.E.; Johnson, M.C.; et al. High-resolution Mass Models of Dwarf Galaxies from LITTLE THINGS. Astro. J. 2015, 149, 180.

189. Spano, M.; Marcelin, M.; Amram, P.; Carignan, C.; Epinat, B.; Hernandez, O. GHASP: An H $\alpha$ kinematic survey of spiral and irregular galaxies-V. Dark matter distribution in 36 nearby spiral galaxies. Mon. Not. Roy. Astro. Soc. 2008, 383, 297-316.

190. Simon, J.D.; Bolatto, A.D.; Leroy, A.; Blitz, L.; Gates, E.L. High-Resolution Measurements of the Halos of Four Dark Matter-Dominated Galaxies: Deviations from a Universal Density Profile. Astrophys. J. 2005, 621, 757-776.

191. de Blok, W.J.G.; Walter, F.; Brinks, E.; Trachternach, C.; Oh, S.H.; Kennicutt, R.C., Jr. High-Resolution Rotation Curves and Galaxy Mass Models from THINGS. Astro. J. 2008, 136, 2648-2719. 
192. Martinsson, T.P.K.; Verheijen, M.A.W.; Westfall, K.B.; Bershady, M.A.; Andersen, D.R.; Swaters, R.A. The DiskMass Survey. VII. The distribution of luminous and dark matter in spiral galaxies. Astron. Astrophys. 2013, 557, A131

193. Simon, J.D.; Bolatto, A.D.; Leroy, A.; Blitz, L. High-Resolution Measurements of the Dark Matter Halo of NGC 2976: Evidence for a Shallow Density Profile. Astrophys. J. 2003, 596, 957-981.

194. Adams, J.J.; Gebhardt, K.; Blanc, G.A.; Fabricius, M.H.; Hill, G.J.; Murphy, J.D.; van den Bosch, R.C.E.; van de Ven, G. The Central Dark Matter Distribution of NGC 2976. Astrophys. J. 2012, 745, 92.

195. Adams, J.J.; Simon, J.D.; Fabricius, M.H.; van den Bosch, R.C.E.; Barentine, J.C.; Bender, R.; Gebhardt, K.; Hill, G.J.; Murphy, J.D.; Swaters, R.A.; et al. Dwarf Galaxy Dark Matter Density Profiles Inferred from Stellar and Gas Kinematics. Astrophys. J. 2014, 789, 63.

196. Oman, K.A.; Navarro, J.F.; Fattahi, A.; Frenk, C.S.; Sawala, T.; White, S.D.M.; Bower, R.; Crain, R.A.; Furlong, M.; Schaller, M.; et al. The unexpected diversity of dwarf galaxy rotation curves. Mon. Not. Roy. Astro. Soc. 2015, 452, 3650-3665.

197. Evans, N.W.; An, J.; Walker, M.G. Cores and cusps in the dwarf spheroidals. Mon. Not. Roy. Astro. Soc. 2009, 393, L50-L54.

198. Wolf, J.; Bullock, J.S. Dark Matter Concentrations and a Search for Cores in Milky Way Dwarf Satellites. arXiv 2012, arXiv:1203.4240.

199. Hayashi, K.; Chiba, M. Probing Non-spherical Dark Halos in the Galactic Dwarf Galaxies. Astrophys. J. 2012, $755,145$.

200. Richardson, T.; Fairbairn, M. Analytical solutions to the mass-anisotropy degeneracy with higher order Jeans analysis: A general method. Mon. Not. Roy. Astro. Soc. 2013, 432, 3361-3380.

201. Jardel, J.R.; Gebhardt, K. The Dark Matter Density Profile of the Fornax Dwarf. Astrophys. J. 2012, 746, 89.

202. Breddels, M.A.; Helmi, A.; van den Bosch, R.C.E.; van de Ven, G.; Battaglia, G. Orbit-based dynamical models of the Sculptor dSph galaxy. Mon. Not. Roy. Astro. Soc. 2013, 433, 3173-3189.

203. Jardel, J.R.; Gebhardt, K. Variations in a Universal Dark Matter Profile for Dwarf Spheroidals. Astrophys. J. Lett. 2013, 775, L30.

204. Jardel, J.R.; Gebhardt, K.; Fabricius, M.H.; Drory, N.; Williams, M.J. Measuring Dark Matter Profiles Non-Parametrically in Dwarf Spheroidals: An Application to Draco. Astrophys. J. 2013, 763, 91.

205. Battaglia, G.; Helmi, A.; Tolstoy, E.; Irwin, M.; Hill, V.; Jablonka, P. The Kinematic Status and Mass Content of the Sculptor Dwarf Spheroidal Galaxy. Astrophys. J. Lett. 2008, 681, L13.

206. Walker, M.G.; Peñarrubia, J. A Method for Measuring (Slopes of) the Mass Profiles of Dwarf Spheroidal Galaxies. Astrophys. J. 2011, 742, 20.

207. Agnello, A.; Evans, N.W. A Virial Core in the Sculptor Dwarf Spheroidal Galaxy. Astrophys. J. Lett. 2012, 754, L39.

208. Amorisco, N.C.; Evans, N.W. Dark matter cores and cusps: The case of multiple stellar populations in dwarf spheroidals. Mon. Not. Roy. Astro. Soc. 2012, 419, 184-196.

209. Sand, D.J.; Treu, T.; Smith, G.P.; Ellis, R.S. The Dark Matter Distribution in the Central Regions of Galaxy Clusters: Implications for Cold Dark Matter. Astrophys. J. 2004, 604, 88-107.

210. Newman, A.B.; Treu, T.; Ellis, R.S.; Sand, D.J.; Richard, J.; Marshall, P.J.; Capak, P.; Miyazaki, S. The Distribution of Dark Matter Over Three Decades in Radius in the Lensing Cluster Abell 611. Astrophys. J. 2009, 706, 1078-1094.

211. Newman, A.B.; Treu, T.; Ellis, R.S.; Sand, D.J. The Dark Matter Distribution in A383: Evidence for a Shallow Density Cusp from Improved Lensing, Stellar Kinematic, and X-ray Data. Astrophys. J. Lett. 2011, 728, L39.

212. Newman, A.B.; Treu, T.; Ellis, R.S.; Sand, D.J. The Density Profiles of Massive, Relaxed Galaxy Clusters. II. Separating Luminous and Dark Matter in Cluster Cores. Astrophys. J. 2013, 765, 25.

213. Del Popolo, A. The flat density profiles of massive, and relaxed galaxy clusters. J. Cosmo. Astrop. Phys 2014, 7, 019.

214. Donnarumma, A.; Ettori, S.; Meneghetti, M.; Gavazzi, R.; Fort, B.; Moscardini, L.; Romano, A.; Fu, L.; Giordano, F.; Radovich, M.; et al. Abell 611. II. X-ray and strong lensing analyses. Astron. Astrophys. 2011, 528, A73.

215. Dahle, H.; Hannestad, S.; Sommer-Larsen, J. The Density Profile of Cluster-Scale Dark Matter Halos. Astrophys. J. Lett. 2003, 588, L73-L76. 
216. Gavazzi, R.; Fort, B.; Mellier, Y.; Pelló, R.; Dantel-Fort, M. A radial mass profile analysis of the lensing cluster MS 2137.3-2353. Astron. Astrophys. 2003, 403, 11-27.

217. Sand, D.J.; Treu, T.; Ellis, R.S. The Dark Matter Density Profile of the Lensing Cluster MS 2137-23: A Test of the Cold Dark Matter Paradigm. Astrophys. J. Lett. 2002, 574, L129-L133.

218. Del Popolo, A. The Mass and temperature functions in a moving barrier model. Mon. Not. R. Astron. Soc. 2002, 337, 529.

219. Del Popolo, A.; Hiotelis, N.; Penarrubia, J. A theoretical study of the luminosity temperature relation for clusters of galaxies. Astrophys. J. 2005, 628, 76.

220. Ettori, S.; Fabian, A.C.; Allen, S.W.; Johnstone, R.M. Deep inside the core of Abell 1795: The Chandra view. Mon. Not. Roy. Astro. Soc. 2002, 331, 635-648.

221. Lewis, A.D.; Buote, D.A.; Stocke, J.T. Chandra Observations of A2029: The Dark Matter Profile Down to below $0.01 r_{\text {vir }}$ in an Unusually Relaxed Cluster. Astrophys. J. 2003, 586, 135-142.

222. Arabadjis, J.S.; Bautz, M.W.; Garmire, G.P. Chandra Observations of the Lensing Cluster EMSS 1358+6245: Implications for Self-interacting Dark Matter. Astrophys. J. 2002, 572, 66-78.

223. Schmidt, R.W.; Allen, S.W. The dark matter haloes of massive, relaxed galaxy clusters observed with Chandra. Mon. Not. Roy. Astro. Soc. 2007, 379, 209-221.

224. Kuzio de Naray, R.; Spekkens, K. Do Baryons Alter the Halos of Low Surface Brightness Galaxies? Astrophys. J. Lett. 2011, 741, L29.

225. Van den Bosch, F.C.; Robertson, B.E.; Dalcanton, J.J.; de Blok, W.J.G. Constraints on the Structure of Dark Matter Halos from the Rotation Curves of Low Surface Brightness Galaxies. Astro. J. 2000, 119, 1579-1591.

226. Power, C.; Navarro, J.F.; Jenkins, A.; Frenk, C.S.; White, S.D.M.; Springel, V.; Stadel, J.; Quinn, T. The inner structure of $\Lambda \mathrm{CDM}$ haloes-I. A numerical convergence study. Mon. Not. Roy. Astro. Soc. 2003, 338, 14-34.

227. De Blok, W.J.G.; McGaugh, S.S.; Bosma, A.; Rubin, V.C. Mass Density Profiles of Low Surface Brightness Galaxies. Astrophys. J. Lett. 2001, 552, L23-L26.

228. Borriello, A.; Salucci, P. The dark matter distribution in disc galaxies. Mon. Not. Roy. Astro. Soc. 2001, 323, 285-292.

229. Diemand, J.; Moore, B.; Stadel, J. Convergence and scatter of cluster density profiles. Mon. Not. Roy. Astro. Soc. 2004, 353, 624-632.

230. Sommer-Larsen, J.; Dolgov, A. Formation of Disk Galaxies: Warm Dark Matter and the Angular Momentum Problem. Astrophys. J. 2001, 551, 608-623.

231. Hu, W.; Barkana, R.; Gruzinov, A. Fuzzy Cold Dark Matter: The Wave Properties of Ultralight Particles. Phys. Rev. Lett. 2000, 85, 1158-1161.

232. Goodman, J. Repulsive dark matter. New Astron. 2000, 5, 103-107.

233. Peebles, P.J.E. Fluid Dark Matter. Astrophys. J. Lett. 2000, 534, L127-L129.

234. Kaplinghat, M.; Knox, L.; Turner, M.S. Annihilating Cold Dark Matter. Phys. Rev. Lett. 2000, 85, $3335-3338$.

235. Cen, R. Decaying Cold Dark Matter Model and Small-Scale Power. Astrophys. J. Lett. 2001, 546, L77-L80.

236. Spergel, D.N.; Steinhardt, P.J. Observational Evidence for Self-Interacting Cold Dark Matter. Phys. Rev. Lett. 2000, 84, 3760-3763.

237. Zentner, A.R.; Bullock, J.S. Halo Substructure and the Power Spectrum. Astrophys. J. 2003, 598, 49-72.

238. Buchdahl, H.A. Non-linear Lagrangians and cosmological theory. Mon. Not. Roy. Astro. Soc. 1970, 150, 1.

239. Starobinsky, A.A. A new type of isotropic cosmological models without singularity. Phys. Lett. B 1980, 91, 99-102.

240. Bengochea, G.R.; Ferraro, R. Dark torsion as the cosmic speed-up. Phys. Rev. D 2009, 79, 124019.

241. Linder, E.V. Einstein's other gravity and the acceleration of the Universe. Phys. Rev. D 2010, 81, 127301.

242. Dent, J.B.; Dutta, S.; Saridakis, E.N. f(T) gravity mimicking dynamical dark energy. Background and perturbation analysis. J. Cosmo. Astrop. Phys 2011, 2011, 009.

243. Zheng, R.; Huang, Q.G. Growth factor in f(T) gravity. J. Cosmo. Astrop. Phys 2011, 2011, 002.

244. Milgrom, M. A modification of the Newtonian dynamics - Implications for galaxies. Astrophys. J. 1983, 270, 371-389.

245. Milgrom, M. A modification of the Newtonian dynamics as a possible alternative to the hidden mass hypothesis. Astrophys. J. 1983, 270, 365-370.

246. Capozziello, S.; De Laurentis, M. The dark matter problem from $\mathrm{f}(\mathrm{R})$ gravity viewpoint. Annalen der Physik 2012, 524, 545-578. 
247. El-Zant, A.; Shlosman, I.; Hoffman, Y. Dark Halos: The Flattening of the Density Cusp by Dynamical Friction. Astrophys. J. 2001, 560, 636-643.

248. El-Zant, A.A.; Hoffman, Y.; Primack, J.; Combes, F.; Shlosman, I. Flat-cored Dark Matter in Cuspy Clusters of Galaxies. Astrophys. J. Lett. 2004, 607, L75-L78.

249. Le Delliou, M.; Henriksen, R.N.; MacMillan, J.D. Black holes and galactic density cusps-I. Radial orbit cusps and bulges. Mon. Not. Roy. Astro. Soc. 2011, 413, 1633-1642.

250. Le Delliou, M.; Henriksen, R.N.; MacMillan, J.D. Black holes and galactic density cusps. Spherically symmetric anisotropic cusps. Astron. Astrophys. 2010, 522, A28.

251. Le Delliou, M.; Henriksen, R.N.; MacMillan, J.D. Black holes and galactic density cusps. From black hole to bulge. Astron. Astrophys. 2011, 526, A13.

252. Nusser, A. Self-similar spherical collapse with non-radial motions. Mon. Not. Roy. Astro. Soc. 2001, 325, 1397-1401.

253. Hiotelis, N. Density profiles in a spherical infall model with non-radial motions. Astron. Astrophys. 2002, 382, 84-91.

254. Le Delliou, M.; Henriksen, R.N. Non-radial motion and the NFW profile. Astron. Astrophys. 2003, 408, 27-38.

255. Ascasibar, Y.; Yepes, G.; Gottlöber, S.; Müller, V. On the physical origin of dark matter density profiles. Mon. Not. Roy. Astro. Soc. 2004, 352, 1109-1120.

256. Williams, L.L.R.; Babul, A.; Dalcanton, J.J. Investigating the Origins of Dark Matter Halo Density Profiles. Astrophys. J. 2004, 604, 18-39.

257. Del Popolo, A.; Pace, F.; Lima, J.A.S. Extended Spherical Collapse and the Accelerating Universe. Int. J. Mod. Phys. D 2013, 22, 1350038.

258. Del Popolo, A.; Pace, F.; Lima, J.A.S. Spherical collapse model with shear and angular momentum in dark energy cosmologies. Mon. Not. Roy. Astro. Soc. 2013, 430, 628-637.

259. Del Popolo, A.; Pace, F.; Maydanyuk, S.P.; Lima, J.A.S.; Jesus, J.F. Shear and rotation in Chaplygin cosmology. Phys. Rev. D 2013, 87, 043527.

260. Del Popolo, A.; Ercan, E.N.; Xia, Z. Ellipsoidal Collapse and Previrialization. Astro. J. 2001, 122, 487-495.

261. Del Popolo, A.; Gambera, M. Substructure effects on the collapse of density perturbations. Astron. Astrophys. 1997, 321, 691-695.

262. Cardone, V.F.; Leubner, M.P.; Del Popolo, A. Spherical galaxy models as equilibrium configurations in non-extensive statistics. Mon. Not. Roy. Astro. Soc. 2011, 414, 2265-2274.

263. Del Popolo, A. Some improvements to the spherical collapse model. Astron. Astrophys. 2006, 454, 17-26.

264. Del Popolo, A. On the evolution of aspherical perturbations in the universe: An analytical model. Astron. Astrophys. 2002, 387, 759.

265. Mimoso, J.P.; Le Delliou, M.; Mena, F.C. Separating expansion from contraction in spherically symmetric models with a perfect fluid: Generalization of the Tolman-Oppenheimer-Volkoff condition and application to models with a cosmological constant. Phys. Rev. D 2010, 81, 123514.

266. Mimoso, J.P.; Le Delliou, M.; Mena, F.C. Local conditions separating expansion from collapse in spherically symmetric models with anisotropic pressures. Phys. Rev. D 2013, 88, 043501.

267. Le Delliou, M.; Mena, F.C.; Mimoso, J.P. Role of shell crossing on the existence and stability of trapped matter shells in spherical inhomogeneous $\Lambda$-CDM models. Phys. Rev. D 2011, 83, 103528.

268. Le Delliou, M.; Mimoso, J.P.; Mena, F.C.; Fontanini, M.; Guariento, D.C.; Abdalla, E. Separating expansion and collapse in general fluid models with heat flux. Phys. Rev. D 2013, 88, 027301.

269. Del Popolo, A.; Gambera, M. Peak mass in large-scale structure and dynamical friction. Astron. Astrophys. 1996, 308, 373-375.

270. Blumenthal, G.R.; Faber, S.M.; Flores, R.; Primack, J.R. Contraction of dark matter galactic halos due to baryonic infall. Astrophys. J. 1986, 301, 27-34.

271. Gnedin, O.Y.; Kravtsov, A.V.; Klypin, A.A.; Nagai, D. Response of Dark Matter Halos to Condensation of Baryons: Cosmological Simulations and Improved Adiabatic Contraction Model. Astrophys. J. 2004, $616,16-26$.

272. Spedicato, E.; Bodon, E.; Del Popolo, A.; Mahdavi-Amiri, N. ABS Methods and ABSPACK for Linear Systems and Optimization, a Review. arXiv 2002, arXiv:0209241.

273. Del Popolo, A.; Kroupa, P. Density profiles of dark matter haloes on galactic and cluster scales. Astron. Astrophys. 2009, 502, 733-747. 
274. Del Popolo, A. A theoretical study of the mass-temperature relation for clusters of galaxies. Mon. Not. Roy. Astro. Soc. 2002, 336, 81-90.

275. Del Popolo, A.; Gambera, M. Non radial motions and the shapes and the abundance of clusters of galaxies. Astron. Astrophys. 2000, 357, 809-815.

276. Colafrancesco, S.; Antonuccio-Delogu, V.; Del Popolo, A. On the Dynamical Origin of Bias in Clusters of Galaxies. Astrophys. J. 1995, 455, 32.

277. Del Popolo, A. Numerical tests of dynamical friction in gravitational inhomogeneous systems. Astron. Astrophys. 2003, 406, 1-6.

278. Navarro, J.F.; Eke, V.R.; Frenk, C.S. The cores of dwarf galaxy haloes. Mon. Not. Roy. Astro. Soc. 1996, 283, L72-L78.

279. Gelato, S.; Sommer-Larsen, J. On DDO 154 and cold dark matter halo profiles. Mon. Not. Roy. Astro. Soc. $1999,303,321-328$.

280. Read, J.I.; Gilmore, G. Mass loss from dwarf spheroidal galaxies: The origins of shallow dark matter cores and exponential surface brightness profiles. Mon. Not. Roy. Astro. Soc. 2005, 356, 107-124.

281. Ma, C.P.; Boylan-Kolchin, M. Are Halos of Collisionless Cold Dark Matter Collisionless? Phys. Rev. Lett. 2004, 93, 021301.

282. Nipoti, C.; Treu, T.; Ciotti, L.; Stiavelli, M. Galactic cannibalism and cold dark matter density profiles. Mon. Not. Roy. Astro. Soc. 2004, 355, 1119-1124.

283. Romano-Díaz, E.; Shlosman, I.; Hoffman, Y.; Heller, C. Erasing Dark Matter Cusps in Cosmological Galactic Halos with Baryons. Astrophys. J. Lett. 2008, 685, L105.

284. Romano-Díaz, E.; Shlosman, I.; Heller, C.; Hoffman, Y. Dissecting Galaxy Formation. I. Comparison Between Pure Dark Matter and Baryonic Models. Astrophys. J. 2009, 702, 1250-1267.

285. Cole, D.R.; Dehnen, W.; Wilkinson, M.I. Weakening dark matter cusps by clumpy baryonic infall. Mon. Not. Roy. Astro. Soc. 2011, 416, 1118-1134.

286. Inoue, S.; Saitoh, T.R. Cores and revived cusps of dark matter haloes in disc galaxy formation through clump clusters. Mon. Not. Roy. Astro. Soc. 2011, 418, 2527-2531.

287. Nipoti, C.; Binney, J. Early flattening of dark matter cusps in dwarf spheroidal galaxies. Mon. Not. Roy. Astro. Soc. 2015, 446, 1820-1828.

288. Pontzen, A.; Governato, F. Cold dark matter heats up. Nature 2014, 506, 171-178.

289. Teyssier, R.; Pontzen, A.; Dubois, Y.; Read, J.I. Cusp-core transformations in dwarf galaxies: Observational predictions. Mon. Not. Roy. Astro. Soc. 2013, 429, 3068-3078.

290. Gnedin, O.Y.; Zhao, H. Maximum feedback and dark matter profiles of dwarf galaxies. Mon. Not. Roy. Astro. Soc. 2002, 333, 299-306.

291. Garrison-Kimmel, S.; Rocha, M.; Boylan-Kolchin, M.; Bullock, J.S.; Lally, J. Can feedback solve the too-big-to-fail problem? Mon. Not. Roy. Astro. Soc. 2013, 433, 3539-3546.

292. Wadsley, J.W.; Stadel, J.; Quinn, T. Gasoline: A flexible, parallel implementation of TreeSPH. New Astron. 2004, 9, 137-158.

293. Katz, N.; White, S.D.M. Hierarchical galaxy formation - Overmerging and the formation of an X-ray cluster. Astrophys. J. 1993, 412, 455-478.

294. Stinson, G.; Seth, A.; Katz, N.; Wadsley, J.; Governato, F.; Quinn, T. Star formation and feedback in smoothed particle hydrodynamic simulations-I. Isolated galaxies. Mon. Not. Roy. Astro. Soc. 2006, 373, 1074-1090.

295. Stinson, G.S.; Brook, C.; Macciò, A.V.; Wadsley, J.; Quinn, T.R.; Couchman, H.M.P. Making Galaxies In a Cosmological Context: The need for early stellar feedback. Mon. Not. Roy. Astro. Soc. 2013, 428, 129-140.

296. Pontzen, A.; Governato, F. How supernova feedback turns dark matter cusps into cores. Mon. Not. Roy. Astro. Soc. 2012, 421, 3464-3471.

297. Ceverino, D.; Klypin, A. The Role of Stellar Feedback in the Formation of Galaxies. Astrophys. J. 2009, 695, 292-309.

298. Oñorbe, J.; Boylan-Kolchin, M.; Bullock, J.S.; Hopkins, P.F.; Kereš, D.; Faucher-Giguère, C.A.; Quataert, E.; Murray, N. Forged in FIRE: Cusps, cores and baryons in low-mass dwarf galaxies. Mon. Not. Roy. Astro. Soc. 2015, 454, 2092-2106.

299. González-Samaniego, A.; Colín, P.; Avila-Reese, V.; Rodríguez-Puebla, A.; Valenzuela, O. Simulations of Isolated Dwarf Galaxies Formed in Dark Matter Halos with Different Mass Assembly Histories. Astrophys. J. 2014, 785, 58. 
300. Pontzen, A.; Governato, F. Flattening Dark Matter Cusps with Supernova Feedback: A Physical Model. Available online: http://astro.dur.ac.uk/Gal2011/uploads/gal2011_durham_talk_Pontzen.pdf (accessed on 8 December 2016)

301. Peñarrubia, J.; Pontzen, A.; Walker, M.G.; Koposov, S.E. The Coupling between the Core/Cusp and Missing Satellite Problems. Astrophys. J. Lett. 2012, 759, L42.

302. Maxwell, A.J.; Wadsley, J.; Couchman, H.M.P. The Energetics of Cusp Destruction. Astrophys. J. 2015, 806, 229.

303. Revaz, Y.; Jablonka, P. The dynamical and chemical evolution of dwarf spheroidal galaxies with GEAR. Astron. Astrophys. 2012, 538, A82.

304. Sawala, T.; Frenk, C.S.; Fattahi, A.; Navarro, J.F.; Bower, R.G.; Crain, R.A.; Dalla Vecchia, C.; Furlong, M.; Helly, J.C.; Jenkins, A.; et al. Local Group galaxies emerge from the dark. arXiv 2014, arXiv:1412.2748.

305. Ferrero, I.; Abadi, M.G.; Navarro, J.F.; Sales, L.V.; Gurovich, S. The dark matter haloes of dwarf galaxies: A challenge for the $\Lambda$ cold dark matter paradigm? Mon. Not. Roy. Astro. Soc. 2012, 425, 2817-2823.

306. Papastergis, E.; Giovanelli, R.; Haynes, M.P.; Shankar, F. Is there a "too big to fail" problem in the field? Astron. Astrophys. 2015, 574, A113.

307. Choi, E.; Ostriker, J.P.; Naab, T.; Oser, L.; Moster, B.P. The impact of mechanical AGN feedback on the formation of massive early-type galaxies. Mon. Not. Roy. Astro. Soc. 2015, 449, 4105-4116.

308. Laporte, C.F.P.; Peñarrubia, J. Under the sword of Damocles: Plausible regeneration of dark matter cusps at the smallest galactic scales. Mon. Not. Roy. Astro. Soc. 2015, 449, L90-L94.

309. Laporte, C.F.P.; White, S.D.M. The redistribution of matter in the cores of galaxy clusters. Mon. Not. Roy. Astro. Soc. 2015, 451, 1177-1189.

310. Koposov, S.E.; Yoo, J.; Rix, H.W.; Weinberg, D.H.; Macciò, A.V.; Escudé, J.M. A Quantitative Explanation of the Observed Population of Milky Way Satellite Galaxies. Astrophys. J. 2009, 696, 2179-2194.

311. Kravtsov, A. Dark Matter Substructure and Dwarf Galactic Satellites. Adv. Astron. 2010, 2010, 281913.

312. Behroozi, P.S.; Conroy, C.; Wechsler, R.H. A Comprehensive Analysis of Uncertainties Affecting the Stellar Mass-Halo Mass Relation for $0<\mathrm{z}<4$. Astrophys. J. 2010, 717, 379-403.

313. Moster, B.P.; Somerville, R.S.; Maulbetsch, C.; van den Bosch, F.C.; Macciò, A.V.; Naab, T.; Oser, L. Constraints on the Relationship between Stellar Mass and Halo Mass at Low and High Redshift. Astrophys. J. 2010, 710, 903-923.

314. Madau, P.; Shen, S.; Governato, F. Dark Matter Heating and Early Core Formation in Dwarf Galaxies. Astrophys. J. Lett. 2014, 789, L17.

315. Noguchi, M. Clumpy star-forming regions as the origin of the peculiar morphology of high-redshift galaxies. Nature 1998, 392, 253.

316. Noguchi, M. Early Evolution of Disk Galaxies: Formation of Bulges in Clumpy Young Galactic Disks. Astrophys. J. 1999, 514, 77-95.

317. Immeli, A.; Samland, M.; Westera, P.; Gerhard, O. Subgalactic Clumps at High Redshift: A Fragmentation Origin? Astrophys. J. 2004, 611, 20-25.

318. Immeli, A.; Samland, M.; Gerhard, O.; Westera, P. Gas physics, disk fragmentation, and bulge formation in young galaxies. Astron. Astrophys. 2004, 413, 547-561.

319. Bournaud, F.; Elmegreen, B.G.; Elmegreen, D.M. Rapid Formation of Exponential Disks and Bulges at High Redshift from the Dynamical Evolution of Clump-Cluster and Chain Galaxies. Astrophys. J. 2007, $670,237-248$.

320. Agertz, O.; Teyssier, R.; Moore, B. Disc formation and the origin of clumpy galaxies at high redshift. Mon. Not. Roy. Astro. Soc. 2009, 397, L64-L68.

321. Aumer, M.; Burkert, A.; Johansson, P.H.; Genzel, R. The Structure of Gravitationally Unstable Gas-rich Disk Galaxies. Astrophys. J. 2010, 719, 1230-1243.

322. Ceverino, D.; Dekel, A.; Bournaud, F. High-redshift clumpy discs and bulges in cosmological simulations. Mon. Not. Roy. Astro. Soc. 2010, 404, 2151-2169.

323. Ceverino, D.; Dekel, A.; Mandelker, N.; Bournaud, F.; Burkert, A.; Genzel, R.; Primack, J. Rotational support of giant clumps in high-z disc galaxies. Mon. Not. Roy. Astro. Soc. 2012, 420, 3490-3520.

324. Toomre, A. On the gravitational stability of a disk of stars. Astrophys. J. 1964, 139, 1217-1238.

325. Krumholz, M.R.; Dekel, A. Survival of star-forming giant clumps in high-redshift galaxies. Mon. Not. Roy. Astro. Soc. 2010, 406, 112-120. 
326. Macciò, A.V.; Stinson, G.; Brook, C.B.; Wadsley, J.; Couchman, H.M.P.; Shen, S.; Gibson, B.K.; Quinn, T. Halo Expansion in Cosmological Hydro Simulations: Toward a Baryonic Solution of the Cusp/Core Problem in Massive Spirals. Astrophys. J. Lett. 2012, 744, L9.

327. Del Popolo, A.; Pace, F. The Cusp/Core problem: Supernovae feedback versus the baryonic clumps and dynamical friction model. Astrophys. Space Sci. 2016, 361, 162.

328. Del Popolo, A. On the dark matter haloes inner structure and galaxy morphology. Astrophys. Space Sci. 2016, 361, 222.

329. Weidner, C.; Kroupa, P.; Pflamm-Altenburg, J.; Vazdekis, A. The galaxy-wide IMF of dwarf late-type to massive early-type galaxies. Mon. Not. R. Astron. Soc. 2013, 436, 3309.

330. Colín, P.; Avila-Reese, V.; Valenzuela, O. Substructure and Halo Density Profiles in a Warm Dark Matter Cosmology. Astrophys. J. 2000, 542, 622-630.

331. Polisensky, E.; Ricotti, M. Constraints on the Dark Matter Particle Mass from the Number of Milky Way Satellites. Phys. Rev. D 2011, 83, 043506.

332. Lovell, M.R.; Eke, V.; Frenk, C.S.; Gao, L.; Jenkins, A.; Theuns, T.; Wang, J.; White, S.D.M.; Boyarsky, A.; Ruchayskiy, O. The haloes of bright satellite galaxies in a warm dark matter universe. Mon. Not. Roy. Astro. Soc. 2012, 420, 2318-2324.

333. Angulo, R.E.; Hahn, O.; Abel, T. The warm dark matter halo mass function below the cut-off scale. Mon. Not. Roy. Astro. Soc. 2013, 434, 3337-3347.

334. Kuzio de Naray, R.; Martinez, G.D.; Bullock, J.S.; Kaplinghat, M. The Case Against Warm or Self-Interacting Dark Matter as Explanations for Cores in Low Surface Brightness Galaxies. Astrophys. J. Lett. 2010, 710, L161-L166.

335. Wu, X.; Kroupa, P. Galactic rotation curves, the baryon-to-dark-halo-mass relation and space-time scale invariance. Mon. Not. R. Astron. Soc. 2015, 446, 330-344.

336. Dalal, N.; Kochanek, C.S. Direct Detection of Cold Dark Matter Substructure. Astrophys. J. 2002, 572, $25-33$.

337. Fadely, R.; Keeton, C.R. Near-infrared K and L' Flux Ratios in Six Lensed Quasars. Astro. J. 2011, 141, 101.

338. Fadely, R.; Keeton, C.R. Substructure in the lens HE 0435-1223. Mon. Not. Roy. Astro. Soc. 2012, 419, $936-951$.

339. Macciò, A.V.; Paduroiu, S.; Anderhalden, D.; Schneider, A.; Moore, B. Cores in warm dark matter haloes: A Catch 22 problem. Mon. Not. Roy. Astro. Soc. 2012, 424, 1105-1112.

340. Schneider, A.; Anderhalden, D.; Macciò, A.V.; Diemand, J. Warm dark matter does not do better than cold dark matter in solving small-scale inconsistencies. Mon. Not. Roy. Astro. Soc. 2014, 441, L6-L10.

341. Narayanan, V.K.; Spergel, D.N.; Davé, R.; Ma, C.P. Constraints on the Mass of Warm Dark Matter Particles and the Shape of the Linear Power Spectrum from the Ly $\alpha$ Forest. Astrophys. J. Lett. 2000, 543, L103-L106.

342. Burkert, A. The Structure and Evolution of Weakly Self-interacting Cold Dark Matter Halos. Astrophys. J. Lett. 2000, 534, L143-L146.

343. Rocha, M.; Peter, A.H.G.; Bullock, J.S.; Kaplinghat, M.; Garrison-Kimmel, S.; Oñorbe, J.; Moustakas, L.A. Cosmological simulations with self-interacting dark matter-I. Constant-density cores and substructure. Mon. Not. Roy. Astro. Soc. 2013, 430, 81-104.

344. Newman, A.B.; Treu, T.; Ellis, R.S.; Sand, D.J.; Nipoti, C.; Richard, J.; Jullo, E. The Density Profiles of Massive, Relaxed Galaxy Clusters. I. The Total Density Over Three Decades in Radius. Astrophys. J. 2013, 765, 24.

345. Peter, A.H.G.; Rocha, M.; Bullock, J.S.; Kaplinghat, M. Cosmological simulations with self-interacting dark matter-II. Halo shapes versus observations. Mon. Not. Roy. Astro. Soc. 2013, 430, 105-120.

346. Clowe, D.; Bradač, M.; Gonzalez, A.H.; Markevitch, M.; Randall, S.W.; Jones, C.; Zaritsky, D. A Direct Empirical Proof of the Existence of Dark Matter. Astrophys. J. Lett. 2006, 648, L109-L113.

347. Randall, S.W.; Markevitch, M.; Clowe, D.; Gonzalez, A.H.; Bradač, M. Constraints on the Self-Interaction Cross Section of Dark Matter from Numerical Simulations of the Merging Galaxy Cluster 1E 0657-56. Astrophys. J. 2008, 679, 1173-1180.

348. Dawson, W.A.; Wittman, D.; Jee, M.J.; Gee, P.; Hughes, J.P.; Tyson, J.A.; Schmidt, S.; Thorman, P.; Bradač, M.; Miyazaki, S.; et al. Discovery of a Dissociative Galaxy Cluster Merger with Large Physical Separation. Astrophys. J. Lett. 2012, 747, L42.

349. Yoshida, N.; Springel, V.; White, S.D.M.; Tormen, G. Weakly Self-interacting Dark Matter and the Structure of Dark Halos. Astrophys. J. Lett. 2000, 544, L87-L90.

350. Davé, R.; Spergel, D.N.; Steinhardt, P.J.; Wandelt, B.D. Halo Properties in Cosmological Simulations of Self-interacting Cold Dark Matter. Astrophys. J. 2001, 547, 574-589. 
351. Harko, T. Bose-Einstein condensation of dark matter solves the core/cusp problem. J. Cosmo. Astrop. Phys 2011, 2011, 022.

352. Schive, H.Y.; Chiueh, T.; Broadhurst, T. Cosmic structure as the quantum interference of a coherent dark wave. Nat. Phys. 2014, 10, 496-499.

353. Robles, V.H.; Matos, T. Flat central density profile and constant dark matter surface density in galaxies from scalar field dark matter. Mon. Not. Roy. Astro. Soc. 2012, 422, 282-289.

354. Riess, A.G.; Filippenko, A.V.; Challis, P.; Clocchiatti, A.; Diercks, A.; Garnavich, P.M.; Gilliland, R.L.; Hogan, C.J.; Jha, S.; Kirshner, R.P.; et al. Observational Evidence from Supernovae for an Accelerating Universe and a Cosmological Constant. Astro. J. 1998, 116, 1009-1038.

355. Perlmutter, S.; Aldering, G.; Goldhaber, G.; Knop, R.A.; Nugent, P.; Castro, P.G.; Deustua, S.; Fabbro, S.; Goobar, A.; Groom, D.E.; et al. Measurements of $\Omega$ and $\Lambda$ from 42 High-Redshift Supernovae. Astrophys. J. 1999, 517, 565-586.

356. Pace, F.; Batista, R.C.; Del Popolo, A. Effects of shear and rotation on the spherical collapse model for clustering dark energy. Mon. Not. R. Astron. Soc. 2014, 445, 648-659.

357. Clifton, T.; Ferreira, P.G.; Padilla, A.; Skordis, C. Modified gravity and cosmology. Phys. Rept. 2012, $513,1-189$.

358. Bull, P.; Akrami, Y.; Adamek, J.; Baker, T.; Bellini, E.; Beltrán Jiménez, J.; Bentivegna, E.; Camera, S.; Clesse, S.; Davis, J.H.; et al. Beyond $\Lambda$ CDM: Problems, solutions, and the road ahead. Phys. Dark Univ. 2016, 12, 56-99.

359. Famaey, B.; McGaugh, S. Modified Newtonian Dynamics (MOND): Observational Phenomenology and Relativistic Extensions. Living Rev. Relativ. 2012, 15, 10.

360. Sanders, R.H.; McGaugh, S.S. Modified Newtonian Dynamics as an Alternative to Dark Matter. Ann. Rev. Astron. Astrophys. 2002, 40, 263-317.

361. Freeman, K.C. On the Disks of Spiral and so Galaxies. Astrophys. J. 1970, 160, 811.

362. Fish, R.A. A Mass-Potential Relationship in Elliptical Galaxies and Some Inferences Concerning the Formation and Evolution of Galaxies. Astrophys. J. 1964, 139, 284.

363. Lelli, F.; McGaugh, S.S.; Schombert, J.M.; Pawlowski, M.S. The Relation between Stellar and Dynamical Surface Densities in the Central Regions of Disk Galaxies. Astrophys. J. 2016, 827, L19.

364. Milgrom, M. Universal MOND Relation Between the Baryonic and 'dynamical' Central Surface Densities of Disc Galaxies. arXiv 2016, arXiv:1607.05103.

365. Famaey, B.; McGaugh, S. The MOND Phenomenology. arXiv 2013, arXiv:1310.4009.

366. Haghi, H.; Bazkiaei, A.E.; Zonoozi, A.H.; Kroupa, P. Declining rotation curves of galaxies as a test of gravitational theory. Mon. Not. R. Astron. Soc. 2016, 458, 4172-4187.

367. Sanders, R.H. A Stratified Framework for Scalar-Tensor Theories of Modified Dynamics. Astrophys. J. 1997, 480, 492-502.

368. Bekenstein, J.D. Relativistic gravitation theory for the modified Newtonian dynamics paradigm. Phys. Rev. D 2004, 70, 083509.

369. Sanders, R.H. A tensor-vector-scalar framework for modified dynamics and cosmic dark matter. Mon. Not. Roy. Astro. Soc. 2005, 363, 459-468.

370. Hashim, N.; De Laurentis, M.; Zainal Abidin, Z.; Salucci, P. Rotation Curve with MOND and Dark Matter Halo Profile for ESO138-G014. arXiv 2014, arXiv:1407.0379.

371. Khoury, J. Alternative to particle dark matter. Phys. Rev. D 2015, 91, 024022.

372. Springel, V.; White, S.D.M.; Frenk, C.S.; Navarro, J.F.; Jenkins, A.; Vogelsberger, M.; Wang, J.; Ludlow, A.; Helmi, A. Prospects for detecting supersymmetric dark matter in the Galactic halo. Nature 2008, 456, 73-76.

373. Diemand, J.; Kuhlen, M.; Madau, P. Dark Matter Substructure and Gamma-Ray Annihilation in the Milky Way Halo. Astrophys. J. 2007, 657, 262-270.

374. Willman, B.; Dalcanton, J.J.; Martinez-Delgado, D.; West, A.A.; Blanton, M.R.; Hogg, D.W.; Barentine, J.C.; Brewington, H.J.; Harvanek, M.; Kleinman, S.J.; et al. A New Milky Way Dwarf Galaxy in Ursa Major. Astrophys. J. Lett. 2005, 626, L85-L88.

375. Belokurov, V.; Zucker, D.B.; Evans, N.W.; Wilkinson, M.I.; Irwin, M.J.; Hodgkin, S.; Bramich, D.M.; Irwin, J.M.; Gilmore, G.; Willman, B.; et al. A Faint New Milky Way Satellite in Bootes. Astrophys. J. Lett. 2006, 647, L111-L114. 
376. Zucker, D.B.; Belokurov, V.; Evans, N.W.; Wilkinson, M.I.; Irwin, M.J.; Sivarani, T.; Hodgkin, S.; Bramich, D.M.; Irwin, J.M.; Gilmore, G.; et al. A New Milky Way Dwarf Satellite in Canes Venatici. Astrophys. J. Lett. 2006, 643, L103-L106.

377. Sakamoto, T.; Hasegawa, T. Discovery of a Faint Old Stellar System at 150 kpc. Astrophys. J. Lett. 2006, 653, L29-L32.

378. Irwin, M.J.; Belokurov, V.; Evans, N.W.; Ryan-Weber, E.V.; de Jong, J.T.A.; Koposov, S.; Zucker, D.B.; Hodgkin, S.T.; Gilmore, G.; Prema, P.; et al. Discovery of an Unusual Dwarf Galaxy in the Outskirts of the Milky Way. Astrophys. J. Lett. 2007, 656, L13-L16.

379. Tollerud, E.J.; Bullock, J.S.; Strigari, L.E.; Willman, B. Hundreds of Milky Way Satellites? Luminosity Bias in the Satellite Luminosity Function. Astrophys. J. 2008, 688, 277-289.

380. Bullock, J.S.; Kravtsov, A.V.; Weinberg, D.H. Reionization and the Abundance of Galactic Satellites. Astrophys. J. 2000, 539, 517-521.

381. Moore, B.; Diemand, J.; Madau, P.; Zemp, M.; Stadel, J. Globular clusters, satellite galaxies and stellar haloes from early dark matter peaks. Mon. Not. Roy. Astro. Soc. 2006, 368, 563-570.

382. Simon, J.D.; Geha, M. The Kinematics of the Ultra-faint Milky Way Satellites: Solving the Missing Satellite Problem. Astrophys. J. 2007, 670, 313-331.

383. Dekel, A.; Silk, J. The origin of dwarf galaxies, cold dark matter, and biased galaxy formation. Astrophys. J. 1986, 303, 39-55.

384. Mori, M.; Yoshii, Y.; Nomoto, K. Dissipative Process as a Mechanism of Differentiating Internal Structures between Dwarf and Normal Elliptical Galaxies in a Cold Dark Matter Universe. Astrophys. J. 1999, $511,585-594$.

385. Mayer, L.; Mastropietro, C.; Wadsley, J.; Stadel, J.; Moore, B. Simultaneous ram pressure and tidal stripping; how dwarf spheroidals lost their gas. Mon. Not. Roy. Astro. Soc. 2006, 369, 1021-1038.

386. Bovill, M.S.; Ricotti, M. Pre-Reionization Fossils, Ultra-Faint Dwarfs, and the Missing Galactic Satellite Problem. Astrophys. J. 2009, 693, 1859-1870.

387. Binggeli, B.; Sandage, A.; Tammann, G.A. Studies of the Virgo Cluster. II-A catalog of 2096 galaxies in the Virgo Cluster area. Astro. J. 1985, 90, 1681-1759.

388. Mateo, M.L. Dwarf Galaxies of the Local Group. Ann. Rev. Astron. Astrophys. 1998, 36, 435-506.

389. Strigari, L.E.; Bullock, J.S.; Kaplinghat, M.; Diemand, J.; Kuhlen, M.; Madau, P. Redefining the Missing Satellites Problem. Astrophys. J. 2007, 669, 676-683.

390. Madau, P.; Diemand, J.; Kuhlen, M. Dark Matter Subhalos and the Dwarf Satellites of the Milky Way. Astrophys. J. 2008, 679, 1260-1271.

391. Zhu, Q.; Marinacci, F.; Maji, M.; Li, Y.; Springel, V.; Hernquist, L. Baryonic impact on the dark matter distribution in Milky Way-sized galaxies and their satellites. Mon. Not. Roy. Astro. Soc. 2016, 458, 1559-1580.

392. Kravtsov, A.V.; Gnedin, O.Y.; Klypin, A.A. The Tumultuous Lives of Galactic Dwarfs and the Missing Satellites Problem. Astrophys. J. 2004, 609, 482-497.

393. Okamoto, T.; Gao, L.; Theuns, T. Mass loss of galaxies due to an ultraviolet background. Mon. Not. Roy. Astro. Soc. 2008, 390, 920-928.

394. Ricotti, M.; Gnedin, N.Y. Formation Histories of Dwarf Galaxies in the Local Group. Astrophys. J. 2005, $629,259-267$.

395. Vera-Ciro, C.A.; Helmi, A.; Starkenburg, E.; Breddels, M.A. Not too big, not too small: The dark haloes of the dwarf spheroidals in the Milky Way. Mon. Not. Roy. Astro. Soc. 2013, 428, 1696-1703.

396. Di Cintio, A.; Knebe, A.; Libeskind, N.I.; Brook, C.; Yepes, G.; Gottlöber, S.; Hoffman, Y. Size matters: The non-universal density profile of subhaloes in SPH simulations and implications for the Milky Way's dSphs. Mon. Not. Roy. Astro. Soc. 2013, 431, 1220-1229.

397. Garrison-Kimmel, S.; Boylan-Kolchin, M.; Bullock, J.S.; Kirby, E.N. Too big to fail in the Local Group. Mon. Not. Roy. Astro. Soc. 2014, 444, 222-236.

398. Brook, C.B.; Di Cintio, A. Expanded haloes, abundance matching and too-big-to-fail in the Local Group. Mon. Not. Roy. Astro. Soc. 2015, 450, 3920-3934.

399. Brook, C.B.; Di Cintio, A. Signatures of dark matter halo expansion in galaxy populations. Mon. Not. Roy. Astro. Soc. 2015, 453, 2133-2143.

400. Papastergis, E.; Shankar, F. An Assessment of the "too Big to Fail" Problem for Field Dwarf Galaxies in View of Baryonic Feedback Effects. arXiv 2015, arXiv:1511.08741. 
401. Sawala, T.; Frenk, C.S.; Fattahi, A.; Navarro, J.F.; Bower, R.G.; Crain, R.A.; Dalla Vecchia, C.; Furlong, M.; Jenkins, A.; McCarthy, I.G.; et al. Bent by baryons: The low-mass galaxy-halo relation. Mon. Not. Roy. Astro. Soc. 2015, 448, 2941-2947.

402. Dutton, A.A.; Macciò, A.V.; Frings, J.; Wang, L.; Stinson, G.S.; Penzo, C.; Kang, X. NIHAO V: Too big does not fail - reconciling the conflict between $\Lambda C D M$ predictions and the circular velocities of nearby field galaxies. Mon. Not. Roy. Astro. Soc. 2016, 457, L74-L78.

403. Del Popolo, A.; Le Delliou, M. A unified solution to the small scale problems of the $\Lambda$ CDM model II: Introducing parent-satellite interaction. J. Cosmo. Astrop. Phys 2014, 2014, 051.

404. Taylor, J.E.; Babul, A. The Dynamics of Sinking Satellites around Disk Galaxies: A Poor Man's Alternative to High-Resolution Numerical Simulations. Astrophys. J. 2001, 559, 716-735.

405. Hiotelis, N.; Del Popolo, A. On the Reliability of Merger-Trees and the Mass-Growth Histories of Dark Matter Haloes. Astrophys. Space Sci. 2006, 301, 167-177.

406. Hiotelis, N.; Del Popolo, A. Anomalous diffusion models for the formation of dark matter haloes. Mon. Not. Roy. Astro. Soc. 2013, 436, 163-178.

407. Öpik, E. Selective absorption of light in space, and the dynamics of the Universe. Bull. Soc. Astr. Russ. 1915, 21,150 .

408. Strigari, L.E.; Frenk, C.S.; White, S.D.M. Dynamical Models for the Sculptor Dwarf Spheroidal in a Lambda CDM Universe. arXiv 2014, arXiv:1406.6079.

409. De Bruijne, J.H.J. Science performance of Gaia, ESA's space-astrometry mission. Astrophys. Space Sci. 2012, $341,31-41$.

410. Takada, M. Subaru Hyper Suprime-Cam Project. AIP Conf. Proc. 2010, 1279, 120-127.

411. Richardson, T.D.; Spolyar, D.; Lehnert, M.D. Plan $\beta$ : Core or cusp? Mon. Not. Roy. Astro. Soc. 2014, 440, 1680-1689.

412. Battaglia, G.; Helmi, A.; Breddels, M. Internal kinematics and dynamical models of dwarf spheroidal galaxies around the Milky Way. New Astron. Rev. 2013, 57, 52-79.

413. Metcalf, R.B.; Amara, A. Small-scale structures of dark matter and flux anomalies in quasar gravitational lenses. Mon. Not. Roy. Astro. Soc. 2012, 419, 3414-3425.

414. Carlberg, R.G.; Grillmair, C.J. Gaps in the GD-1 Star Stream. Astrophys. J. 2013, 768, 171.

(C) 2017 by the authors. Licensee MDPI, Basel, Switzerland. This article is an open access article distributed under the terms and conditions of the Creative Commons Attribution (CC BY) license (http:/ / creativecommons.org/licenses/by/4.0/). 